\title{
A unique adipocyte progenitor population promotes age-related adiposity
}

\section{Qiong Wang ( $\nabla$ qwang@coh.org )}

City of Hope/ Beckman Research Institute https://orcid.org/0000-0003-2224-4287

\section{Guan Wang}

City of Hope/ Beckman Research Institute

\section{Gaoyan Li}

UCLA

\section{Anying Song}

City of Hope/ Beckman Research Institute

\section{Wenting Dai}

City of Hope/ Beckman Research Institute

\section{Nicholas Shamlin}

City of Hope/ Beckman Research Institute Hanjun Qin

Beckman Research Institute, City of Hope

\section{Leonard Medrano}

City of Hope/ Beckman Research Institute

\section{Joan Dow}

City of Hope/ Beckman Research Institute

\section{Aimin Li}

City of Hope/ Beckman Research Institute

\section{Lucy Brown}

City of Hope/ Beckman Research Institute

\section{Brian Armstrong}

Beckman Research Institute at City of Hope Medical Center

\section{Patrick Fueger}

City of Hope

Judith Campisi

Buck Institute

\section{Xiwei Wu}

City of Hope

\section{Lei Jiang}

City of Hope https://orcid.org/0000-0002-8596-556X

\section{Xia Yang}




\section{Biological Sciences - Article}

Keywords: fat mass, aging, adipocyte progenitor cells (APCs), lineage tracing, metabolic disorders

Posted Date: October 1st, 2021

DOl: https://doi.org/10.21203/rs.3.rs-888694/v1

License: @ (i) This work is licensed under a Creative Commons Attribution 4.0 International License. Read Full License 
3 Guan Wang ${ }^{1 \#}$, Gaoyan $\mathrm{Li}^{2 \#}$, Anying Song ${ }^{1}$, Wenting Dai ${ }^{1}$, Nicholas Shamlin ${ }^{1}$, Hanjun Qin $^{3}$,

4 Leonard Medrano ${ }^{4}$, Joan Dow ${ }^{1,5}$, Aimin $\mathrm{Li}^{6}$, Lucy Brown ${ }^{7}$, Brian Armstrong ${ }^{8}$, Patrick $\mathrm{T}$.

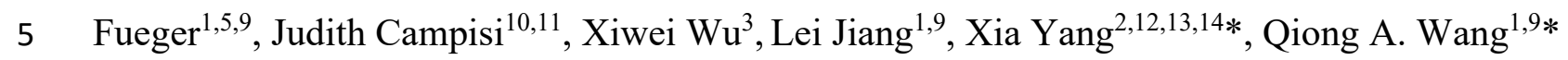

$6 \quad{ }^{1}$ Department of Molecular \& Cellular Endocrinology, Arthur Riggs Diabetes and Metabolism

7 Research Institute, ${ }^{3}$ The Integrative Genomics Core, ${ }^{4}$ Division of Developmental and Translational

8 Diabetes and Endocrinology Research, ${ }^{5}$ Comprehensive Metabolic Phenotyping Core, ${ }^{6}$ Pathology

9 Core of Shared resources, ${ }^{7}$ Analytical Cytometry Core, ${ }^{8}$ Light Microscopy Core, ${ }^{9}$ Comprehensive

10 Cancer Center, Beckman Research Institute, City of Hope Medical Center, Duarte, California 1191010 , USA.

$12{ }^{10}$ Buck Institute for Research on Aging, Novato, California.

$13{ }^{11}$ Biosciences Division, Lawrence Berkeley National Laboratory, Berkeley, California.

$14{ }^{2}$ Department of Integrative Biology and Physiology, ${ }^{12}$ Bioinformatics Interdepartmental Program,

$15{ }^{13}$ Institute for Quantitative and Computational Biosciences, ${ }^{14}$ Molecular Biology Institute, 16 University of California, Los Angeles, Los Angeles, CA 90095 USA

\#These authors contributed equally.

*Correspondence should be addressed to:

21 Qiong (Annabel) Wang, Department of Molecular \& Cellular Endocrinology, City of Hope.

221500 East Duarte Road, Duarte, CA 91010

23 E-mail: qwang@coh.org; Phone: 626-218-6419

Xia Yang, Department of Integrative Biology and Physiology, Los Angeles, CA 90095

26 E-mail: xyang123@ucla.edu; Phone: 310-206-1812

27

28 Conflict of interest: The authors have declared that no conflict of interest exists. 


\section{Abstract}

The average fat mass in adults increases dramatically with age, and older people often suffer

33 from visceral obesity and related adverse metabolic disorders. Unfortunately, how aging leads to

34 fat accumulation is poorly understood. It is known that fat cell (adipocyte) turnover is very low in

35 young mice, similar to that in young humans. Here, we find that mice mimic age-related fat

36 expansion in humans. In vivo lineage tracing shows that massive adipogenesis (the generation of

37 new adipocytes), especially in the visceral fat, is triggered during aging. Thus, in contrast to most

38 types of adult stem cells that exhibit a reduced ability to proliferate and differentiate, the

39 adipogenic potential of adipocyte progenitor cells (APCs) is unlocked by aging. In vivo

40 transplantation and 3D imaging of transplants show that APCs in aged mice cell-autonomously

41 gain high adipogenic capacity. Single-cell RNA sequencing analyses reveal that aging globally

42 remodels APCs. Herein, we identify a novel committed preadipocyte population that is age-

43 specific (CP-A), existing both in mice and humans, with a global activation of proliferation and

44 adipogenesis pathways. CP-A cells display high proliferation and adipogenesis activity, both in

45 vivo and in vitro. Macrophages may regulate the remodeling of APCs and the generation of CP-A

46 cells during aging. Together, these findings define a new fundamental mechanism involved in fat

47 tissue aging and offer prospects for preventing and treating age-related metabolic disorders.

48

$49 \quad 225$ words

50 
52 At the same BMI, older people, on average, tend to have more body fat (white adipose tissue, 53 WAT) than younger adults, especially in the visceral (abdominal) area ${ }^{1,2}$. At the same time, skeletal 54 muscle mass declines progressively with age. These aged-related alterations in body composition 55 are defined as sarcopenic obesity ${ }^{3,4}$. Visceral adiposity accelerates aging by promoting insulin 56 resistance, cardiovascular dysfunction, and many other chronic diseases, significantly shortening 57 both healthspan and lifespan ${ }^{5,6}$. Thus, preventing or reducing WAT accumulation is critical for 58 healthy aging. A recent study shows that older individuals have reduced energy expenditure, 59 indicating a global remodeling of tissue metabolism during aging ${ }^{7}$. Unfortunately, the cellular mechanism of age-related WAT accumulation is still poorly understood.

61

62 WAT regulates numerous hormonal and metabolic processes, and exhibits compositional and 63 phenotypic plasticity ${ }^{8}$. WAT expands via adipocyte hypertrophy (cell enlargement) or 64 adipogenesis (the generation of new adipocytes). We and others generated multiple mouse models 65 that allow in vivo tracking of adipogenesis, and showed that the adipogenesis rate is very low in 66 young adult mice ${ }^{9-11}$, similar to that in young adult humans ${ }^{12}$. During aging, it is known that 67 adipocytes undergo hypertrophy ${ }^{13}$, but it remains unclear if WAT also expands through 68 adipogenesis, which would grant WAT an "unlimited" potential to grow.

Adipocyte progenitor cells (APCs), located within the WAT stroma, are a heterogeneous group

71 of mesenchymal stem cells, including adipocyte stem cells and committed progenitor cells ${ }^{8}$.

72 Adipogenesis involves two steps, the proliferation of APCs and the differentiation of APCs into 73 mature adipocytes. APCs express a set of mesenchymal stem cell surface markers, such as Pdgfra, 
74 Pdgfr $\beta$, CD29, CD34, and Sca $1^{14}$. Recently, single-cell RNA sequencing (scRNA-seq) has 75 provided unprecedented opportunities to define the heterogeneity of murine and human $\mathrm{APCs}^{15-21}$, 76 revealing new subtypes and dynamics under various pathophysiological conditions. in contrast to most adult stem cells that exhibit a reduced ability to proliferate and differentiate ${ }^{22-}$ imaging of transplants, we showed that the APCs in aged mice cell-autonomously gain high adipogenic capacity. scRNA-seq analysis revealed a global transformation of APCs in aged mice and older humans and uncovered a new APC population that is uniquely generated during aging. insights into the cellular mechanism of age-related adiposity.

\section{Results}

\section{Aged-related WAT expansion in mice mimics human sarcopenic obesity}

At 12 (aged) vs. 3 (young) months old, male C57BL/6J mice showed a 56\% increase in body weight (Fig. 1a, b). The age-related body weight gain in male mice was primarily attributed to gain of WAT, as the percentage of fat mass increased $>6$-fold during aging (Fig. 1c), while the 
97 with age (Extended Data Fig. 1a). Bodyweight gain with age was much higher in males than

98 females (Extended Data Fig. 1b, c). Among the two types of fat depots, gonadal WAT (gWAT),

99 a typical type of visceral WAT, had the most significant weight gain with age (4.6-fold) (Fig. 1e),

100 while subcutaneous WAT (sWAT) increased 2.8-fold (Fig. 1f). The aged mice exhibited

101 dramatically reduced oxygen consumption (Fig. 1g), slightly reduced physical activity (Fig. 1h)

102 and total movement (Extended Data Fig. 1d), as well as dramatically reduced energy expenditure

103 (Fig. 1i). Food intake was also reduced in aged mice (Extended Data Fig. 1e). The drop in oxygen

104 consumption and energy expenditure suggested that the aged mice have significantly lower basal

105 metabolism, similar to humans ${ }^{7}$. The decline in basal metabolism causes a positive energy balance

106 (energy intake exceeds expenditure), eventually leads to fat accumulation. Intraperitoneal glucose

107 tolerance tests (GTT) (Fig. 1j, k) and insulin tolerance tests (ITT) (Fig. 1l, m) showed that aged

108 mice have impaired glucose tolerance and are more insulin resistant. Therefore, mice display a

109 pattern of age-related visceral adiposity, accompanied by positive energy balance and insulin

110 resistance, similar to human sarcopenic obesity.

112 WAT accumulates with age through massive adipogenesis.

113 To better understand adipocyte dynamics, we previously developed the AdipoChaser mice for 114 tracking adipogenesis ${ }^{16,17,19,31,32}$. This mouse model is a doxycycline (dox)-based, tet-responsive 115 labeling system for the inducible, permanent labeling of Adiponectin (Adipoq) expressing cells as 116 LacZ+ cells (Extended Data Fig. 1f). To determine if aging is accompanied by adipogenesis, we 117 utilized AdipoChaser-LacZ mice to label all adipocytes as LacZ+ cells in 3-month-old male mice 118 and tracked adipogenesis during early aging (Extended Data Fig. 1g). Consistent with our 119 previous studies, in 6-month-old mice, we observed no new adipocytes (i.e., no lacZ-negative cells) 
120 in the gWAT or sWAT, indicating that the turnover rate of adipocytes is extremely low in young 121 adults (Fig. 1n). In contrast, in 9-month-old mice, clusters of newly generated adipocytes started

122 to appear in gWAT. New adipocytes also started to appear in sWAT but did not yet form clusters.

123 As mice continue aging, in 18-month-old mice, gWAT showed numerous new adipocytes, which

124 formed large clusters; sWAT also had clusters of new adipocytes, which are relatively smaller than

125 gWAT. Adipocyte size was also increased in sWAT and gWAT, confirming the age-related 126 adipocyte hypertrophy ${ }^{21}$ (Fig. 1n). Female mice were found quite different from the males

127 (Extended Data Fig. 1h). In the female gWAT, we observed some adipogenesis in 18-month-old 128 female mice, but the rate is much lower than that in male mice. In the female sWAT, we did not 129 observe any new adipocytes generated during aging. Thus, adipogenesis is the major contributor 130 to age-related gWAT expansion in male mice.

We next tested if aged mice would have a higher adipocyte regeneration rate after inducible adipocyte apoptosis. The FAT-ATTAC mice (fat apoptosis through triggered activation of caspase 8) carry a transgene encoding a caspase 8 protein fused to a dimerization domain under the control 135 of the aP2 adipocyte-specific promoter ${ }^{25}$. We triggered adipocyte apoptosis through 7 days of daily 136 injection of dimerizer, and checked the adipocyte regeneration post dimerization in young and 137 aged mice (Extended Data Fig. 2a). Prior to treatment, the adipocytes in young and aged FAT138 ATTAC mice were viable with positive perilipin staining before dimerization (Extended Data 139 Fig. 2b, c). After dimerization, apoptosis was initiated in every adipocyte, as adipocytes were 140 uniformly dead, with disrupted morphology and negative perilipin staining (Extended Data Fig. $\mathbf{2 b}, \mathbf{c})$. One week after dimerization, gWAT volume was markedly reduced in both young and aged mice (Extended Data Fig. 2b, c). At two-four weeks after dimerization, the aged mice 
143 demonstrated strong adipocyte regeneration with essentially more adipocytes positive for perilipin.

144 gWAT from young mice, while found to have some perilipin-positive adipocytes, still had an 145 abnormal morphology with many lost cells and vacuoles (Extended Data Fig. 2b, c). By four 146 weeks of treatment, the gWAT of aged mice had regained more tissue mass than young mice 147 (Extended Data Fig. 2d). Thus, aged mice exhibit higher adipocyte regeneration capacity, through 148 earlier and faster de novo adipogenesis.

\section{APCs from aged mice cell-autonomously have a high adipogenic capacity in vivo}

Do APCs cell-autonomously undergo adipogenesis during aging? Alternatively, is there a systemic stimulus required for age-related adipogenesis? APCs were reported to be less

153 immunogenic and less subject to rejection than other cell types following transplantation into 154 mice $^{33}$. We then set out to test the in vivo adipogenic ability of APCs from gWAT of young vs. 155 aged male mice through transplantation. An equal number of APCs (Lin-, CD45-CD31-Ter119-) 156 from the stromal vascular fraction (SVF) of gWAT were mixed from 2.5-month-old male CAG157 EGFP mice (GFP+, "young" APCs) and from 12-month-old male Rosa26-loxp-mTomato-stop158 loxp-GFP (Rosa26-mT/mG) mice (Tomato+, "aged" APCs) (Fig. 2a). The cell mixture was 159 transplanted into the sWAT of 2.5-month-old male wide type (WT) mice (Fig. 2b-f, Extended 160 Data Video 1). When the Matrigel transplants were dissected one month later, we observed that more "aged" APCs (Tomato+) had differentiated into adipocytes (Fig. 2b, Extended Data Video

162 1), resulting in a 2.5 -fold more adipocytes and 2.2-fold greater adipocyte volume compared to 163 "young" APCs (GFP+) (Fig. 2c, d). These GFP+ and Tomato+ cells (differentiated from GFP+ or 164 Tomato + APCs) were indeed mature adipocytes, as they have a unilocular morphology (single 165 lipid droplet) (Fig. 2e) and were perilipin positive (Fig. 2f). To further confirm our observation, 
we also transplanted Tomato + APCs from the gWAT of 2.5-month-old and 12-month-old male Rosa26-mT/mG mice separately into the different sides of sWATs of the same male WT mice (Fig. 2g). Four weeks after transplantation, the "aged" APCs had a significantly higher (3 folds) adipogenesis rate, compared to the "young" APCs (Fig. 2h-j, Extended Data Video 2, 3). Thus, the APCs from older mice maintain their high adipogenic potential after transplantation into a “young" environment.

took the reverse approach and transplanted APCs from young mice into aged mice. Equal numbers of "young" Tomato+ APCs from gWAT of 2.5-month-old male Rosa26-mT/mG were transplanted into sWATs of 12-month-old and 2.5-month-old male WT mice (Extended Data Fig. 3a). Four weeks after the transplantation, no significant adipogenesis of the "young" APCs was observed in young or old recipient mice (Extended Data Fig. 3b, Extended Data Video 4, 5). Similarly, when GFP+ APCs from young CAG-EGFP mice were transplanted into aged WT mice (Extended Data Fig. 3c), the age-related systemic stimulation and microenvironment did not trigger significant adipogenesis of the "young" APCs (Extended Data Fig. 3d, Extended Data Video 6, 7).

Altogether, these transplantation results indicate that the "aged" APCs cell-autonomously exhibit greater adipogenic rate in the in vivo setting, regardless of "young" or "aged" microenvironment.

\section{Aging generates a new APC population with a globally activated adipogenic program}

To explore the molecular underpinning of the adipogenic properties of the APCs from aged 
mice, we performed scRNA-seq of the CD45-CD31-Ter119- stromal vascular cells from the gWAT of aged and young mice ( $\mathrm{n}=3$ mice/group) (Extended Data Fig. 4a). Among the 19,534 cells sequenced, 15,194 cells (78\%) were identified as progenitor cells (Extended Data Fig. 4b), based on the expression of classic APC markers CD34, CD29, PDGFR $\alpha$, and Sca-1. While the variation between mice within the same group was minimal (Extended Data Fig. 4c), there were dramatic transcriptomic shifts between young and aged cells (Extended Data Fig. 4d). gene expression profiles to identify five cell clusters (Fig. 3a). Based on recently published scRNA-seq analysis of WAT APCs ${ }^{28,34,35}$, we named these clusters: 1) Adipocyte stem cell (ASC); 2) intermediate adipocyte progenitor (IAP); 3) committed preadipocyte 1 (CP-1); 4) committed preadipocyte 2 (CP-2); 5) committed preadipocyte, age-specific (CP-A). We found that aging significantly remodeled the progenitor cell population, as "aged" cells had "shifted" away from "young" cells, and cells from the two groups had little overlap (Fig. 3b, c). Using Slingshot trajectory analysis, we revealed potential lineage relationships among these APC clusters (Fig. 3d, Extended Data Fig. 4e). Within the "young" progenitor cells, ASC had two developmental trajectories, both through IAP, and terminated as CP-1 and CP-2 (Fig. 3d, Extended Data Fig. 4e). Interestingly, within the "aged' progenitor cells, ASC had one additional, unique developmental trajectory, which terminated as CP-A (Fig. 3d, Extended Data Fig. 4e). Quantifying the percentage of each cell cluster showed that the CP-A population only existed in aged mice, whereas the CP-1 population was dramatically reduced in aged mice (Fig. 3e). These results suggest that the aging process generated a new adipogenic lineage from ASC to CP-A. 
Next, we used CytoTRACE analysis and sorted the order of these APC clusters based on 213 differentiation status as predicted by transcript abundance (Fig. 3f, Extended Data Fig. 4f, g). 214 From stem cells to committed precursor cells, the order of these clusters was: ASC, CP-2, IAP, 215 CP-1, and CP-A. For each type of cluster, the "Young" APCs were always more stem cell-like compared to the "aged" APCs. And among all clusters, the "aged" CP-A cluster was identified as

217 the most committed APC. The mesenchymal progenitor marker Pdgfra was universally expressed 218 in all clusters (Fig. 3g), but each cluster also showed unique markers (Extended Data Fig. 4h). 219 ASC cluster expressed Cd55, Pi16, and Dpp4 (Fig. 3h, Extended Data Fig. 4h, i); IAP cluster 220 expressed common adipocyte progenitor markers (e.g., Pdgfra), and intermediate levels of ASC markers and committed preadipocytes (Extended Data Fig. 4h); CP-1 cluster expressed Apoe, Igfl, and C7 (Fig. 3i, Extended Data Fig. 4h); CP-2 cluster expressed Mfap4, Mgp, and Cilp (Fig. 3j, Extended Data Fig. 4h). CP-A cluster were marked by expression of Thbs 1 and Lifr (Fig. 3k, Extended Data Fig. 4h, j). To determine which cluster(s) within the "aged" APCs undergo active 225 adipogenesis during aging, we assessed the biological pathways enriched in the marker genes of each cluster. Interestingly, many genes involved in the adipogenic pathway is highly upregulated in the CP-A cluster, which is unique to the aged group (Fig. 31). The enrichment of the adipogenic pathway in the $\mathrm{CP}-\mathrm{A}$ cluster indicates that this population is uniquely generated during aging and 229 may underlie age-related adipogenesis.

We then compared our clusters with the previously published APC clusters in WAT. For each of the published APC clusters in the indicated studies, gene module scores were calculated based on a list of significantly enriched marker genes. Burl et al. identified two major APC clusters 234 (ASC1 and ASC2) in gWAT representing different cell states during adipogenesis. In 
235 comparison $^{15}$, the ASC cluster we noted highly overlapped with the Dpp4/Pi16/Cd55+ ASC2 236 cluster. Our CP-1 cluster highly overlapped with the Icam1/Igf1/Adam12+ ASC1 cluster. Our IAP 237 and CP-2 clusters partially overlapped with both ASC1 and ASC2 clusters, and the CP-A cluster 238 partially overlapped with the ASC1 cluster (Extended Data Fig. 5a, b). Sárvári et al. utilized 239 single-nucleus RNA-seq to identify four major APC clusters in gWAT $^{20}$. Our ASC cluster 240 overlapped with the FAP2, FAP3, and FAP4 clusters. IAP, CP-1, CP-2, and CP-A clusters partially 241 overlapped with the FAP1 and FAP2 clusters (Extended Data Fig. 5c, d). Schwalie et al. 242 identified three major APC clusters in $\mathrm{sWAT}^{17}$. Our ASC cluster overlaps extensively with the P1 243 cluster. Our IAP, CP-1, CP-2, and CP-A clusters overlap with the P2 cluster, and partially overlap 244 with the adipogenesis-regulatory cells, the P3 cluster (Extended Data Fig. 5e, f). Taken together, 245 the five major APC clusters we identified shared similarities with previously defined APC 246 populations both in gWAT and sWAT. Despite the similarities, our study uniquely identified the 247 CP-A cluster as an age- and depot-specific population with unique molecular characteristics.

\section{Human visceral WAT shows similar patterns of age-related APC remodeling}

To access the translational relevance of these findings, we set out to verify if humans also have

the same age-specific CP-A population with scRNA-seq. CD45-CD31-CD235a- SVFs from

252 human peripancreatic WAT (5 human samples of different sexes and ages) were used for scRNA253 seq. Among the 24,794 cells sequenced, 20,431 cells (82\%) were identified as progenitor cells 254 (Extended Data Fig. 6a). Unsupervised clustering of gene expression profiles divided these APCs 255 into eight cell clusters (Hu0-Hu7) (Fig. 3m). To compare the identified APC populations from 256 mice with the human clusters, we calculated the ASC and CP-A gene module score as a sum of 257 mouse marker genes (Fig. 3n). Clusters Hu0, 2, 3, 6 were enriched with mouse ASC markers, 
258 while clusters Hu1, 5, 7 were enriched with mouse CP-A markers (Fig. 3n). Thus, both mouse 259 ASC and CP-A similar cells can be identified in human visceral WAT. All progenitor cells showed 260 abundant expression of APC marker PDGFRA (Extended Data Fig. 6b) and PDGFRB (Extended 261 Data Fig. 6c). Human clusters similar to mouse ASC had high expression of the ASC markers 262 CD55 and Pi16+ (Extended Data Fig. 6d, e). Human clusters similar to mouse CP-A had high 263 expression of the CP-A marker LIFR (Extended Data Fig. 6f), and moderately (or partially) 264 expressed $P P A R G, F A B P 4$, and $L P L$ (Extended Data Fig. 6g-i). We then used flow cytometry to 265 analyze the LIFR+ population in human peripancreatic WAT of different age groups. Among the 266 four samples obtained from male donors, we isolated SVFs and enriched APC (CD31-CD45267 CD235a-PDGFR $\alpha+$ cells) (Extended Data Fig. 6j), and found a trend for the LIFR+ population 268 to dramatically increase with age (Fig. 3o). Thus, APCs in human vWAT may undergo similar 269 remodeling during aging, and the human CP-A population may also contribute to sarcopenic 270 obesity.

271

272

273

CP-A population has a high proliferation and adipogenesis rate both in vitro and in vivo 273 Pathway analysis suggested that proliferation-related pathways, such as TGF $\beta^{18}$ and mitotic 274 signaling, are enriched in the "aged" ASCs compared to the "young" ASCs (Fig. 4a, Extended 275 Data Table 1). Moreover, the adipogenesis pathway is highly enriched in the age-specific CP-A 276 population (Fig. 4b, Extended Data Table 2). This analysis suggests that the "aged" ASC and 277 CP-A populations may have increased proliferation and inflammation, and the CP-A population 278 also has increased adipogenesis.

279

We then determined the capacity of total APC to proliferate and differentiate into adipocytes 
281 in 3D culture in vitro (Fig. 4c). The enriched APCs were first mixed with Matrigel and cultured in 282 the growth medium for 72 hours. An adipogenic cocktail was then added to differentiate these cells 283 into adipocytes. Starting with the same number of cells, "aged" APCs proliferated into more cells 284 compared to the "young" APCs (Extended Data Fig. 7a). However, the adipogenesis rate (the 285 percentage of Bodipy+ adipocytes in Tomato+ cells) for both "young" and "aged" APCs were 286 similarly high, close to $100 \%$. The advantage of the 3D culture system is that we do not need the 287 APCs to grow densely and attach prior to adipogenesis. We then added the adipogenic cocktail 16 288 hours later after seeding to the enriched APC populations (Extended Data Fig. 7b). "Aged" APCs 289 had higher (but not significant) adipogenesis rate, and significantly higher proliferation rate, as the total Tomato+ cell number from "aged" APCs was more than 2 folds greater than the "young" 291 APCs (Extended Data Fig. 7b).

292

Flow analysis showed that the LIFR+ (CP-A) population did significantly increase with age (Extended Data Fig. 7c). In light of results from the pathway analysis, we next inquired whether the CP-A population generated during aging has greater capacity in proliferation and differentiation. We next enriched individual APC populations and tested their proliferation and adipogenic capacity in vitro. To minimize stress to cells, a magnetic beads-based negative selection was used to enrich APCs (Lin-, CD31-CD45-Ter119-) from freshly isolated SVFs from the gWAT of young and aged male Rosa26-mT/mG mice. To enrich the ASC population, we isolated the DPP4+ population from APCs. DPP4+ and DPP4- populations were validated by flow analysis (Extended Data Fig. 7d). To enrich the CP-1 and CP-A population, negative selection, depleting DPP4+ ASC, CD9+ CP-2, and IAP populations in APCs were employed. The enriched populations were validated and showed a low percentage of DPP4 and CD9 expression and a high percentage 
of LIFR expression on flow analysis (Extended Data Fig. 7e, f). Interestingly, more DPP4+ APCs from the gWAT of aged male mice switched from CD9- cells to CD9+ cells, suggesting that a certain number of cells in the aged ASC population may have started the adipogenesis program 307 along the trajectory to IAP (Extended Data Fig. 7g). This also validates that aged ASCs are more differentiated than young ASC as suggested by the CytoTRACE analysis (Fig. 3f). The "aged" ASC population had an adipogenesis rate similar to the "young" ASCs, but they had a significantly higher proliferation rate (Fig. 4d, e). We also compared the age-specific CP-A population with the "young" CP-1 population, which shares the highest molecular similarity with the CP-A population according to the scRNA-seq results. Importantly, the CP-A population not only had a more than four folds higher proliferation rate compared to the CP-1 population, but had the highest 314 adipogenesis rate among all populations tested (Fig. 4f, g). Thus, the CP-A population, uniquely generated with age, has the most remarkable capacity for proliferation and differentiation in vitro.

We then enriched APC populations and tested their proliferation and adipogenic capacity in vivo. A large amount of DPP4- APCs (containing all APC populations other than ASC), CP-1, and 319 CP-A populations were collected from freshly isolated SVFs from gWAT of 2.5-month-old and 12-month-old male Rosa26-mT/mG mice, and equal numbers of these enriched "young" and "aged" populations were transplanted into the different sides of the sWATs of male WT mice (Fig. 4h).

322 The "aged" DPP4- APCs grew substantially larger in volume compared to the "young" DPP4323 APCs (Fig. 4i-k, Extended Data Video 8, 9). More notably, the CP-A population grew six times 324 larger in volume compared to the CP-1 population (Fig. 4l-n, Extended Data Video 10, 11), 325 indicating that the CP-A population has a striking ability to proliferate in vivo. Not surprisingly, 326 most of the CP-A population differentiated into adipocytes. 
Taken together, both the in vitro and in vivo results indicate that the CP-A population has a greater capacity for proliferation and adipogenesis compared to the other APC populations.

\section{Enhanced interactions from macrophages to "aged" APCs}

Aging has been reported to alter the immune system in $\mathrm{WAT}^{26-28}$, we next investigated potential immune cell-APC interactions. We performed scRNA-seq of the CD45+ SVFs from the gWAT of aged and young mice ( $\mathrm{n}=3$ mice/group: the same group of mice were used for the APC analysis), which identified 10,486 cells (Extended Data Fig. 8a). Unsupervised clustering of gene expression profiles identified all the major immune cell clusters, including macrophage and M2like macrophage, T cell, B cell, and neutrophil (Extended Data Fig. 8a, b). The variation between mice within the same age group was minimal (Extended Data Fig. 8c), but there was not much mixture of the "aged" cells with the "young" cells in each immune cluster, reflecting a dramatic remodeling of immune cells by aging (Extended Data Fig. 8d). Flow cytometry showed no difference in the percentage of total macrophage or M2-like macrophage in total immune cells isolated from the gWAT of young and aged mice (Extended Data Fig. 8e).

We inferred the interaction frequency between immune cells and APC populations based on ligand-receptor pairs using the CellphoneDB database and the iTALK database, which indicated that the interactions between macrophages and APC populations are dramatically increased in aged mice (Extended Data Fig. 8f). We then analyzed the one-way ligand-receptor communication from immune cells to APC populations. We selected three ligand-receptor groups: adipogenesis

349 (Extended Data Fig. 8g, h), TGF $\beta$ signaling (Extended Data Fig. 8i, j), and insulin signaling 
(Extended Data Fig. 8k, l). Among all the immune cell types, macrophages were found to have increased ligand-receptor pairing with all of the APC populations. We then narrowed down the analysis by selecting one-way ligand-receptor communication only from macrophages to APC populations (Extended Data Fig. 9). More ligand-receptor pairing showed up as we narrowed down the cell types, again, signals that promote adipogenesis (Extended Data Fig. 9a), TGF $\beta$ (Extended Data Fig. 9b), and insulin signaling (Extended Data Fig. 9c), were uniquely increased from the "aged" macrophages to "aged" ASC. Signals sent from the "aged" macrophages to the "aged" CP-A were compared with signaling sent from the "young" macrophages to the "young" CP-1 (Extended Data Fig. 9d-i). Indeed, from the "aged" macrophages to "aged" CP-A, signaling that promotes adipogenesis (Extended Data Fig. 9d, g), and insulin signaling (Extended Data Fig. 9f, i), were increased both in the number of ligand-receptor pairs and the signal intensity. TGF $\beta$ signaling was not altered in the number of ligand-receptor pairs, but many of them did increase in signal intensity in the aged condition (Extended Data Fig. 9e, h). In summary, the increased proliferation and adipogenesis phenotype in the "aged" APC subpopulations is highly likely regulated by local macrophages.

Evidence of macrophage-APC crosstalk suggested possible effects on phenotype. We then enriched APCs and tested if macrophages could promote the phenotype of the "aged" APCs. APCs (Lin-, CD31-CD45-Ter119-) from freshly isolated SVFs from the gWAT of young and aged male Rosa26-mT/mG mice were mixed with macrophages from young and aged male WT mice. Matrigel containing the cell mixture was seeded in the growth medium for 16 hours, then the adipogenic cocktail was added to drive adipocyte differentiation (Extended Data Fig. 10a). Macrophages, regardless of the age of the donor mice, increased the proliferation rate of APCs of 
373 both "young" and "aged" mice. However, co-culture with macrophage did not increase the 374 adipogenesis rate of APCs of both "young" and "aged" mice (Extended Data Fig. 10b). We note 375 that the in vitro co-culture experiment may not capture the role of macrophages in the remodeling 376 of APCs during aging, which may take weeks and months.

377

378 Discussion

379 As a highly plastic organ, WAT alters in volume in adaptation to a variety of physiological and 380 pathological metabolic challenges. We and others have shown that without any metabolic 381 challenge, healthy young adult mice or humans have extremely low adipocyte turnover rates. WAT 382 plasticity under many physiological and pathological challenges has been extensively studied, such 383 as cold exposure and high fat diet feeding ${ }^{9,10}$. However, the expansion of WAT during early aging 384 is surprisingly understudied. In this study, we provide the first cellular and molecular evidence that 385 aging triggers massive adipogenesis of unique APC populations (Extended Data Fig. 10c). aging. As vWAT and sWAT are fundamentally distinct organs with distinct development timelines, molecular signatures, and metabolic functions ${ }^{9,10,29}$, it is not surprising that age-related adiposity 390 happens in the vWAT at a much higher rate. Compared to hypertrophy, which gives WAT only 391 limited potential to expand, adipogenesis is alarming because it grants WAT unlimited potential 392 for growth. Our findings on the age-related adipogenesis, therefore, highlights adipogenesis but 393 not hypertrophy, as the key process to intervene for age-related obesity and other associated 394 diseases. 
Age-related adiposity accelerates aging and shortens life expectancy. Signaling pathways that 397 are known to extend lifespan, e.g., SIRT, FOXO, and JNK, often inhibit adipogenesis (the making 398 of new adipocytes) and decrease WAT mass ${ }^{30-34}$. Conversely, signaling pathways that are known 399 to shorten lifespan, e.g., insulin/IGF-1 and mTOR, often do the opposite ${ }^{35-37}$. Similarly, strategies 400 that promote longevity and youthfulness often inhibit WAT expansion in aged animals and older 401 humans, such as caloric restriction ${ }^{38-40}$ and fasting ${ }^{41}$. These critical studies suggest that inhibiting 402 WAT expansion during aging is closely linked to increased healthspan and lifespan. Our study 403 expands on this and suggests that to limit WAT expansion during aging, it is crucial to understand 404 the underlying mechanism of age-related adipogenesis.

Our in vivo transplant and in vitro differentiation results suggest that "aged" APCs cell407 autonomously have a greater adipogenic capacity, even when disassociated with the aging 408 microenvironment. However, the aging microenvironment is potentially crucial for driving the 409 remodeling of APCs, which may have started during early aging. Dr. James Kirkland's group 410 elegantly showed that APCs could undergo cellular senescence and lose the ability to differentiate 411 into adipocytes ${ }^{42}$. Therefore, their study suggested that cellular senescence contributes to age412 related fat loss, which happens in the very aged population. In this study, we focused on WAT 413 expansion during early and middle-aging. Our study indicates that although some APCs may 414 become senescent and stop undergoing adipogenesis during aging, sufficient APCs are generated 415 during early aging and contribute to the massive adipogenesis we observed in vivo. Interestingly, 416 senescent cells accumulate specifically in vWAT during early aging ${ }^{43-45}$, and we showed that the 417 vWAT has the highest adipogenic rate. It is possible that the senescent microenvironment 418 promotes adipogenesis of APCs in vWAT during early aging. Firstly, cellular senescence 
419 positively correlates with WAT mass. Inducing cellular senescence through genetic manipulation

420 often results in WAT expansion ${ }^{46,47}$; in addition to accumulating during aging, senescent cells also

421 accumulate in obesity, especially in the vWAT ${ }^{48-53}$, which has active adipogenesis. Secondly,

422 senescent cells are known to stimulate adult stem cell proliferation and differentiation or tumor

423 proliferation ${ }^{54-56}$. Lastly, inflammatory signals are known to promote adipogenesis ${ }^{57-59}$; senescent

424 cells may promote adipogenesis through the senescence-associated secretory phenotype ${ }^{60-62}$,

425 which contains numerous pro-inflammatory cytokines. Currently, we are trying to determine if

426 cellular senescence promotes adipogenesis by remodeling the APC niche.

427

428

429 into APC heterogeneity in a fat depot-specific manner ${ }^{63,64}$. Around four major APC subpopulations had been identified, including 1) adipose stem cells (ASCs) ${ }^{15,16,18}$, which is also identified in our 431 study as the ASC population; 2) committed preadipocytes (adipocyte progenitors) ${ }^{15,16,18}$, which is 432 also identified in our study as the CP-1 and CP-2 populations; 3 ) fibro-inflammatory progenitors 433 (FIPs) ${ }^{16,65,66}$; and 4) adipogenesis regulators (Aregs). Aregs inhibit adipogenesis and were found 434 in both adult sWAT ${ }^{17}$ and aged $\mathrm{sWAT}^{21}$. Our ASC cluster bears a close resemblance to the gWAT 435 PDGFR $\beta+$ stromal subpopulation ${ }^{16}$. The gene signatures of our ASC cells are molecularly similar 436 to those of APCs (adipogenic progenitor cells) and CPs (committed preadipocytes), the two 437 adipogenic clusters defined previously ${ }^{16}$. In the same study, another anti-adipogenic subpopulation, 438 or FIPs (fibro-inflammatory progenitors), was also defined in gWAT. In our gWAT scRNA-seq 439 data, the FIP markers were not apparently enriched within the defined clusters, likely because the 440 previous study analyzed pre-selected PDGFR $\beta+$ cells, which represent a fraction of SVF cells so 441 that FIPs are not resolved by scRNA-seq in our samples. In the present study, we identified a new, 
442 committed preadipocyte population, the CP-A population, which is uniquely generated in the 443 vWAT of aged mice and older humans. Importantly, the CP-A cells displayed superior capacity, 444 compared to all other known APC subpopulations, to proliferate and differentiate into mature 445 adipocytes, both in vitro and in vivo.

446

447

448

449

450

451

452

453

454

455

456

457

458

459

460

461

462

463

464

The CP-A markers LIFR and Thbs1 distinguish these cells from other committed preadipocytes. LIFR forms a heterodimer with gp130 and induces JAK-STAT3 signaling pathway $^{67}$, which has been demonstrated to promote adipogenesis from APCs ${ }^{68}$. Pathway Enrichment analysis also indicates upregulated JAK-STAT3 pathway in the CP-A population, suggesting the activation of LIFR signaling. The other CP-A marker, Thbs1 is recognized as an adipokine that is associated with obesity and insulin resistance ${ }^{69}$. Adding exogenous Thbs 1 was found to promote the proliferation of $3 \mathrm{~T} 3-\mathrm{L} 1$ cells $^{70}$. Thbs1 also activates TGF $\beta 1$ signaling ${ }^{71}$, which might promote a positive interacting loop between ASC and CP-A populations. Thus, LIFR and Thbs1 may play essential roles in promoting CP-A proliferation and differentiation.

In addition to the discovery of the new age-specific CP-A population, our scRNA-seq data showed that the other APC populations also had little overlap between the aged and young groups, indicating that the aging process significantly transformed all APC sub-populations. Indeed, we found that the "aged" ASC population had a higher proliferation rate, which is also essential for age-related adipogenesis. Interestingly, a large proportion of aged ASCs also expressed CD9, a representative marker of IAP and CP-2 populations. These findings suggest that aged ASCs might have started the differentiation program and have a higher tendency to differentiate into mature adipocytes. CD9 is a marker of the FIP population ${ }^{16}$. Thus, CP-2 is likely to be the analogous 
465 population of FIPs. CD9+ progenitors have been reported to control adipose tissue fibrosis ${ }^{72}$. As

466 vWAT fibrosis progresses during aging ${ }^{73}$, the ASC to CP-2 transition could contribute to age-

467 related adipose fibrosis and metabolic dysfunction. Considering the presence of anti-adipogenic

468 stromal cell subpopulations as identified in multiple fat depots ${ }^{16,17,21,65,66}$, it is not excluded that

469 the age-dependent gWAT hyperplastic expansion can be ascribed to the dysregulation of

470 suppressive mechanisms governed by anti-adipogenic cells such as FIPs or Aregs, which also

471 requires further investigation in the future.

472

473 Besides APCs, various immune cell types have been reported to regulate APC differentiation

474 and adipose homeostasis under different conditions in mice and humans ${ }^{26,74-76}$. Similar to APCs,

475 there was a significant shift in nearly all the immune cells in aged gWAT. We also showed that

476 adipose tissue macrophages (ATMs) account for the majority cell type among the immune cells.

477 These ATMs are Lyve1+, indicating that they might resemble the previously reported perivascular-

478 like macrophages $(\mathrm{PVM})^{20}$. They also have a high expression level of Retnla, a specific marker of

479 adipose infiltrating M2 macrophages ${ }^{77}$. This suggests that adipose inflammation in age-related

480 obesity is different from that in high fat diet-induced obesity. In accordance with this, these ATMs

481 did not express Trem2, a marker of previously identified lipid-associated macrophages (LAM)

482 emerging in gWAT after high fat diet feeding ${ }^{75}$. Additionally, CD44 signaling was increased in

483 the aged ASC population, which has been reported to mediate the interaction with macrophage ${ }^{78}$.

484 Co-culture with ATMs did not increase the differentiation of APCs, but did promote cell growth, 485 suggesting that ATMs regulate APCs during aging. Ligand-receptor analysis suggests that the cell486 cell communications between macrophages and APCs increase in aged mice compared to other

487 immune cell types. There would be a positive feedback loop within ATMs and APCs in mediating 
age-related adipogenesis. Besides, a recent paper demonstrates that adipose-resident macrophages promote adipocyte hypertrophy through PDGFc ${ }^{79}$. Our scRNA-seq analysis indicates that Pdgfc is highly expressed in the MAC2 population, which may indicate that hyperplasia rather than hypertrophy is the dominant factor to control age-related adiposity. Thus, how macrophages remodel APCs during aging needs further investigation.

In conclusion, we found that massive adipogenesis is triggered during aging, and largely contributes to age-related visceral adiposity. We then discovered a new, age-specific APC subpopulation: the CP-A cells, which cell-autonomously mastered high proliferation and adipogenesis capacity. Future studies will need to determine how the CP-A population emerges during aging and whether the immune-regulation of adipose tissue plasticity during aging plays a role in this process. It is worth further investigating the crosstalk between different immune cell types and all the APC subpopulations to better understand the molecular and cellular dynamics in adipose remodeling to pinpoint key regulatory cell types and genes. Other than immune cells, other key regulators discussed above, such as cellular senescence, adipose fibrosis, may also remodel the APC subpopulations and promote the generation of the CP-A population. Most importantly, effective strategies eliminating the CP-A population or inhibiting its proliferation and differentiation will prevent the development of visceral adiposity during aging, improve agerelated metabolic disorders, and eventually increase healthspan and lifespan.

\section{Author contributions}

QAW and G.W. designed the experiments. QAW, G.W., G.L., and X.Y. wrote the manuscript. G.W., A.S., and Q.A.W. handled all the mouse experiments. G.W. and J.D. performed mouse MRI 
511 and metabolic cage studies. G.W., Q.A.W., A.S., and N.S. performed $\beta$-gal staining. G.W. and 512 N.S. performed 2D immunofluorescence staining and imaging. G.W. and W.D. prepared the 513 primary SVFs for scRNA-seq, H.Q. and X.W. conducted the scRNA-seq experiments. G.L. and 514 X.Y. analyzed the scRNA-seq data. A.L. performed tissue embedding and sectioning. G.W. and 515 N.S. performed all the in vivo transplantations. G.W. performed all in vitro culture and co-culture 516 experiments. G.W. and B.A. performed all the 3D immunofluorescence imaging. G.W. and L.B. 517 performed FACS and analysis. P.F., J.C. and L.J. contributed to experimental design and 518 discussion.

520 Acknowledgments

521 The authors are grateful to Philipp Scherer (UTSW), Rana Gupta (UTSW), Bart Deplancke 522 (EPFL), Mengle Shao (Shanghai Pasteur), Li Ye (Scripps), Debbie Thurmond (COH), Jeffrey 523 Isenberg $(\mathrm{COH})$, and other members of the Arthur Riggs Diabetes and Metabolism Research 524 Institute at City of Hope for discussions and comments. The authors thank Jianying Zhang at the 525 City of Hope Biostatistics and Mathematical Oncology Core for statistical analysis guidance. The 526 authors thank the City of Hope Animal Resource Center, Integrative Genomics Core, 527 Comprehensive Metabolic Phenotyping Core, Light Microscopy Core, Pathology (Solid Tumor) 528 Core (supported by NIH P30CA033572), Analytical Cytometry Core, and City of Hope 529 Comprehensive Cancer Center for guidance and assistance for experiments. This study was 530 directly supported by National Institutes of Health grants R56AG063854 and R01AG063854 to 531 Q.A.W.. Q.A.W. was also supported by National Institutes of Health grants R01HD096152, 532 R01DK128907, the American Diabetes Association Junior Faculty Development Award 1-19533 JDF-023, and the Caltech-COH Initiative Award. 


\section{Reference}

5351 Flegal, K. M., Kruszon-Moran, D., Carroll, M. D., Fryar, C. D. \& Ogden, C. L. Trends in 536

537 Obesity Among Adults in the United States, 2005 to 2014. JAMA 315, 2284-2291,

5382 Gallagher, D. et al. Healthy percentage body fat ranges: an approach for developing 539 guidelines based on body mass index. Am J Clin Nutr 72, 694-701, 540 doi:10.1093/ajcn/72.3.694 (2000).

5413 Stenholm, S. et al. Sarcopenic obesity: definition, cause and consequences. Curr Opin Clin 542 Nutr Metab Care 11, 693-700, doi:10.1097/MCO.0b013e328312c37d (2008).

5434 Roh, E. \& Choi, K. M. Health Consequences of Sarcopenic Obesity: A Narrative Review. $544 \quad$ Frontiers in Endocrinology 11, doi:10.3389/fendo.2020.00332 (2020).

5455 Karakelides, H., Irving, B. A., Short, K. R., O'Brien, P. \& Nair, K. S. Age, obesity, and sex $546 \quad$ effects on insulin sensitivity and skeletal muscle mitochondrial function. Diabetes 59, 895479 97, doi:10.2337/db09-0591 (2010).

5486 Decaria, J. E., Sharp, C. \& Petrella, R. J. Scoping review report: obesity in older adults. Int 549 J Obes (Lond) 36, 1141-1150, doi:10.1038/ijo.2012.29 (2012).

5507 Pontzer, H. et al. Daily energy expenditure through the human life course. Science 373, $551 \quad$ 808-812, doi:10.1126/science.abe5017 (2021).

5528 Ghaben, A. L. \& Scherer, P. E. Adipogenesis and metabolic health. Nat Rev Mol Cell Biol, 553 doi:10.1038/s41580-018-0093-z (2019).

5549 Wang, Q. A., Tao, C., Gupta, R. K. \& Scherer, P. E. Tracking adipogenesis during white 555 adipose tissue development, expansion and regeneration. Nat Med 19, 1338-1344, 556 doi:10.1038/nm.3324 (2013). 
55710 Vishvanath, L. et al. Pdgfr $\beta+$ Mural Preadipocytes Contribute to Adipocyte Hyperplasia 558 Induced by High-Fat-Diet Feeding and Prolonged Cold Exposure in Adult Mice. Cell Metabolism 23, 350-359, doi:http://dx.doi.org/10.1016/j.cmet.2015.10.018 (2016).

56011 Wang, Q. A. et al. Reversible De-differentiation of Mature White Adipocytes into 561 Preadipocyte-like Precursors during Lactation. Cell Metabolism 28, 282-288.e283, 562 doi:https://doi.org/10.1016/j.cmet.2018.05.022 (2018).

56312 Spalding, K. L. et al. Dynamics of fat cell turnover in humans. Nature 453, 783-787, 564 doi:10.1038/nature06902 (2008).

56513 Palmer, A. K. \& Kirkland, J. L. Aging and adipose tissue: potential interventions for 566 diabetes and regenerative medicine. Experimental Gerontology 86, 97-105, 567 doi:https://doi.org/10.1016/j.exger.2016.02.013 (2016).

56814 Hepler, C., Vishvanath, L. \& Gupta, R. K. Sorting out adipocyte precursors and their role $569 \quad$ in physiology and disease. Genes Dev 31, 127-140, doi:10.1101/gad.293704.116 (2017).

57015 Burl, R. B. et al. Deconstructing Adipogenesis Induced by $\beta 3$-Adrenergic Receptor 571 572

57316 Hepler, C. et al. Identification of functionally distinct fibro-inflammatory and adipogenic 574 stromal subpopulations in visceral adipose tissue of adult mice. eLife 7, 575 doi:10.7554/eLife.39636 (2018).

57617 Schwalie, P. C. et al. A stromal cell population that inhibits adipogenesis in mammalian 577 fat depots. Nature 559, 103-108, doi:10.1038/s41586-018-0226-8 (2018).

57818 Merrick, D. et al. Identification of a mesenchymal progenitor cell hierarchy in adipose 579 tissue. Science 364, doi:10.1126/science.aav2501 (2019). 
58019 Vijay, J. et al. Single-cell analysis of human adipose tissue identifies depot and disease 581 specific cell types. Nat Metab 2, 97-109, doi:10.1038/s42255-019-0152-6 (2020).

58220 Sárvári, A. K. et al. Plasticity of Epididymal Adipose Tissue in Response to Diet-Induced 583 Obesity at Single-Nucleus Resolution. Cell Metab 33, 437-453.e435, 584 doi:10.1016/j.cmet.2020.12.004 (2021).

58521 Nguyen, H. P. et al. Aging-dependent regulatory cells emerge in subcutaneous fat to inhibit 586 587 588 adipogenesis. Developmental cell 56, 1437-1451.e1433, doi:10.1016/j.devcel.2021.03.026 (2021). opportunities. Nat Med 20, 870-880, doi:10.1038/nm.3651 (2014).

59023 Ren, R., Ocampo, A., Liu, G. H. \& Izpisua Belmonte, J. C. Regulation of Stem Cell Aging 591 by Metabolism and Epigenetics. Cell Metab 26, 460-474, doi:10.1016/j.cmet.2017.07.019 592 (2017).

59324 Ambrosi, T. H. et al. Aged skeletal stem cells generate an inflammatory degenerative niche. $594 \quad$ Nature, doi:10.1038/s41586-021-03795-7 (2021).

59525 Pajvani, U. B. et al. Fat apoptosis through targeted activation of caspase 8: a new mouse 596 model of inducible and reversible lipoatrophy. Nat Med 11, 797-803, doi:10.1038/nm1262 $597 \quad(2005)$

59826 Camell, C. D. et al. Aging Induces an Nlrp3 Inflammasome-Dependent Expansion of 599 Adipose B Cells That Impairs Metabolic Homeostasis. Cell Metab 30, 1024-1039 e1026, 600 doi:10.1016/j.cmet.2019.10.006 (2019).

60127 Camell, C. D. et al. Inflammasome-driven catecholamine catabolism in macrophages 602 blunts lipolysis during ageing. Nature 550, 119-123, doi:10.1038/nature24022 (2017). 
60328 Gao, H. et al. Age-Induced Reduction in Human Lipolysis: A Potential Role for Adipocyte 604 Noradrenaline Degradation. Cell Metab 32, 1-3, doi:10.1016/j.cmet.2020.06.007 (2020).

60529 Wang, Q. A. et al. Distinct regulatory mechanisms governing embryonic versus adult 606 adipocyte maturation. Nature cell biology 17, 1099 (2015).

60730 Picard, F. et al. Sirt1 promotes fat mobilization in white adipocytes by repressing PPAR608 gamma. Nature 429, 771-776, doi:10.1038/nature02583 (2004).

60931 Kanfi, Y. et al. SIRT6 protects against pathological damage caused by diet-induced obesity. $610 \quad$ Aging cell 9, 162-173 (2010).

61132 Armoni, M. et al. FOXO1 represses peroxisome proliferator-activated receptor-gamma1 612 and -gamma2 gene promoters in primary adipocytes. A novel paradigm to increase insulin 613 sensitivity. The Journal of biological chemistry 281, 19881-19891, 614 doi:10.1074/jbc.M600320200 (2006).

61533 Wang, M. C., Bohmann, D. \& Jasper, H. JNK Extends Life Span and Limits Growth by 616 Antagonizing Cellular and Organism-Wide Responses to Insulin Signaling. Cell 121, 115617 125, doi:https://doi.org/10.1016/j.cell.2005.02.030 (2005).

61834 Roichman, A. et al. SIRT6 Overexpression Improves Various Aspects of Mouse 619 Healthspan. J Gerontol A Biol Sci Med Sci 72, 603-615, doi:10.1093/gerona/glw152 (2017). 62035 Bluher, M., Kahn, B. B. \& Kahn, C. R. Extended longevity in mice lacking the insulin 621 receptor in adipose tissue. Science 299, 572-574, doi:10.1126/science.1078223 (2003).

62236 Um, S. H. et al. Absence of S6K1 protects against age- and diet-induced obesity while 623 enhancing insulin sensitivity. Nature 431, 200, doi:10.1038/nature02866

624 https://www.nature.com/articles/nature02866\#supplementary-information (2004). 
62537 Lamming, D. W. \& Sabatini, D. M. A Central role for mTOR in lipid homeostasis. Cell 626 Metab 18, 465-469, doi:10.1016/j.cmet.2013.08.002 (2013).

62738 Barzilai, N., Banerjee, S., Hawkins, M., Chen, W. \& Rossetti, L. Caloric restriction 628 reverses hepatic insulin resistance in aging rats by decreasing visceral fat. J Clin Invest 101, 629

63039 Redman, L. M. et al. Metabolic Slowing and Reduced Oxidative Damage with Sustained 631 632 633

40 Wu, Z. et al. Dietary Restriction Extends Lifespan through Metabolic Regulation of Innate 634 Immunity. Cell Metab 29, 1192-1205 e1198, doi:10.1016/j.cmet.2019.02.013 (2019).

63541 Brandhorst, S. et al. A Periodic Diet that Mimics Fasting Promotes Multi-System 636

$42 \mathrm{Xu}, \mathrm{M}$. et al. Targeting senescent cells enhances adipogenesis and metabolic function in 639 old age. eLife 4, e12997, doi:10.7554/eLife.12997 (2015).

64043 van Deursen, J. M. The role of senescent cells in ageing. Nature 509, 439, 641 doi:10.1038/nature13193 (2014).

64244 Childs, B. G., Durik, M., Baker, D. J. \& van Deursen, J. M. Cellular senescence in aging 643 and age-related disease: from mechanisms to therapy. Nature Medicine 21, 1424, 644 doi:10.1038/nm.4000 (2015).

64545 Baker, D. J. et al. Naturally occurring p16Ink4a-positive cells shorten healthy lifespan. 646 Nature 530, 184, doi:10.1038/nature 16932

647 https://www.nature.com/articles/nature16932\#supplementary-information (2016). 
64846 Minamino, T. et al. A crucial role for adipose tissue p53 in the regulation of insulin 649 resistance. Nature Medicine 15, 1082, doi:10.1038/nm.2014

650 https://www.nature.com/articles/nm.2014\#supplementary-information (2009).

65147 Chen, Y. W., Harris, R. A., Hatahet, Z. \& Chou, K. M. Ablation of XP-V gene causes 652 adipose tissue senescence and metabolic abnormalities. Proc Natl Acad Sci U S A 112, 653 E4556-4564, doi:10.1073/pnas.1506954112 (2015).

65448 Wang, C. Y. et al. Obesity increases vascular senescence and susceptibility to ischemic 655 injury through chronic activation of Akt and mTOR. Sci Signal 2, ra11, 656 doi:10.1126/scisignal.2000143 (2009).

65749 Tchkonia, T. et al. Fat tissue, aging, and cellular senescence. Aging cell 9, 667-684 (2010). 65850 Villaret, A. et al. Adipose tissue endothelial cells from obese human subjects: differences 659 among depots in angiogenic, metabolic, and inflammatory gene expression and cellular 660 senescence. Diabetes (2010).

66151 Palmer, A. K. et al. Cellular Senescence in Type 2 Diabetes: A Therapeutic Opportunity. 662 Diabetes 64, 2289-2298, doi:10.2337/db14-1820 (2015).

66352 Schafer, M. J. et al. Exercise Prevents Diet-Induced Cellular Senescence in Adipose Tissue. $664 \quad$ Diabetes 65, 1606-1615, doi:10.2337/db15-0291 (2016).

66553 Shirakawa, K. et al. Obesity accelerates T cell senescence in murine visceral adipose tissue. 666 The Journal of Clinical Investigation 126, 4626-4639, doi:10.1172/JCI88606 (2016).

66754 Krtolica, A., Parrinello, S., Lockett, S., Desprez, P. Y. \& Campisi, J. Senescent fibroblasts 668 promote epithelial cell growth and tumorigenesis: a link between cancer and aging. Proc $669 \quad$ Natl Acad Sci U S A 98, 12072-12077, doi:10.1073/pnas.211053698 (2001). 
67055 Campisi, J. Aging, cellular senescence, and cancer. Annual review of physiology 75, 685-

$671 \quad$ 705, doi:10.1146/annurev-physiol-030212-183653 (2013).

67256 Ruhland, M. K. et al. Stromal senescence establishes an immunosuppressive 673 microenvironment that drives tumorigenesis. Nat Commun 7, 11762, 674 doi:10.1038/ncomms11762 (2016).

67557 Asterholm, I. W. et al. Adipocyte inflammation is essential for healthy adipose tissue 676 expansion and remodeling. Cell metabolism 20, 103-118 (2014).

67758 Lackey, D. E. \& Olefsky, J. M. Regulation of metabolism by the innate immune system. $678 \quad$ Nature Reviews Endocrinology 12, 15 (2016).

67959 Hotamisligil, G. S. Inflammation, metaflammation and immunometabolic disorders. $680 \quad$ Nature 542, 177, doi:10.1038/nature21363

681 https://www.nature.com/articles/nature21363\#supplementary-information (2017).

68260 Rodier, F. et al. Persistent DNA damage signalling triggers senescence-associated 683 inflammatory cytokine secretion. Nat Cell Biol 11, 973-979, doi:10.1038/ncb1909 (2009).

68461 He, S. \& Sharpless, N. E. Senescence in Health and Disease. Cell 169, 1000-1011, 685 doi:10.1016/j.cell.2017.05.015 (2017).

68662 Sapieha, P. \& Mallette, F. A. Cellular Senescence in Postmitotic Cells: Beyond Growth 687 Arrest. Trends in cell biology (2018).

68863 Rondini, E. A. \& Granneman, J. G. Single cell approaches to address adipose tissue stromal 689 cell heterogeneity. Biochem J 477, 583-600, doi:10.1042/bcj20190467 (2020).

69064 Ferrero, R., Rainer, P. \& Deplancke, B. Toward a Consensus View of Mammalian 691 Adipocyte Stem and Progenitor Cell Heterogeneity. Trends in Cell Biology 30, 937-950, 692 doi:https://doi.org/10.1016/j.tcb.2020.09.007 (2020). 
69365 Shao, M. et al. Pathologic HIF1 $\alpha$ signaling drives adipose progenitor dysfunction in 694 obesity. Cell Stem Cell 28, 685-701.e687, doi:10.1016/j.stem.2020.12.008 (2021).

69566 Shan, B. et al. Perivascular mesenchymal cells control adipose-tissue macrophage accrual 696 in obesity. Nat Metab 2, 1332-1349, doi:10.1038/s42255-020-00301-7 (2020).

69767 White, U. A. \& Stephens, J. M. The gp130 receptor cytokine family: regulators of 698 adipocyte development and function. Curr Pharm Des 17, 340-346, 699 doi:10.2174/138161211795164202 (2011).

70068 Richard, A. J. \& Stephens, J. M. Emerging roles of JAK-STAT signaling pathways in 701 adipocytes. Trends Endocrinol Metab 22, 325-332, doi:10.1016/j.tem.2011.03.007 (2011).

70269 Varma, V. et al. Thrombospondin-1 is an adipokine associated with obesity, adipose 703 inflammation, and insulin resistance. Diabetes 57, 432-439, doi:10.2337/db07-0840 (2008).

70470 Kong, P. et al. Thrombospondin-1 regulates adiposity and metabolic dysfunction in diet705 706 induced obesity enhancing adipose inflammation and stimulating adipocyte proliferation. Am J Physiol Endocrinol Metab 305, E439-450, doi:10.1152/ajpendo.00006.2013 (2013).

70771 Isenberg, J. S., Martin-Manso, G., Maxhimer, J. B. \& Roberts, D. D. Regulation of nitric 708 oxide signalling by thrombospondin 1: implications for anti-angiogenic therapies. Nat Rev 709 Cancer 9, 182-194, doi:10.1038/nrc2561 (2009).

71072 Marcelin, G. et al. A PDGFRalpha-Mediated Switch toward CD9(high) Adipocyte 711 Progenitors Controls Obesity-Induced Adipose Tissue Fibrosis. Cell Metab 25, 673-685, 71373 Marcelin, G., Silveira, A. L. M., Martins, L. B., Ferreira, A. V. \& Clement, K. Deciphering 714 715 the cellular interplays underlying obesity-induced adipose tissue fibrosis. J Clin Invest 129, 4032-4040, doi:10.1172/JCI129192 (2019). 
71674 Hildreth, A. D. et al. Single-cell sequencing of human white adipose tissue identifies new

717 cell states in health and obesity. Nat Immunol 22, 639-653, doi:10.1038/s41590-021$718 \quad 00922-4(2021)$.

71975 Jaitin, D. A. et al. Lipid-Associated Macrophages Control Metabolic Homeostasis in a 720 Trem2-Dependent Manner. Cell 178, 686-698 e614, doi:10.1016/j.cell.2019.05.054 (2019).

72176 LaMarche, N. M. et al. Distinct iNKT Cell Populations Use IFNgamma or ER Stress-

722 Induced IL-10 to Control Adipose Tissue Homeostasis. Cell Metab 32, 243-258 e246, 723 doi:10.1016/j.cmet.2020.05.017 (2020).

72477 Titos, E. et al. Resolvin D1 and its precursor docosahexaenoic acid promote resolution of 725 adipose tissue inflammation by eliciting macrophage polarization toward an M2-like 726 phenotype. J Immunol 187, 5408-5418, doi:10.4049/jimmunol.1100225 (2011).

72778 Lee, Y. H., Petkova, A. P. \& Granneman, J. G. Identification of an adipogenic niche for 728 adipose tissue remodeling and restoration. Cell Metab 18, 355-367, 729 doi:10.1016/j.cmet.2013.08.003 (2013).

73079 Cox, N. et al. Diet-regulated production of PDGFcc by macrophages controls energy $731 \quad$ storage. Science 373, doi:10.1126/science.abe9383 (2021). 


\section{Method}

\section{Mice}

Mice were maintained in a $12 \mathrm{~h}$ dark/light cycle and housed in groups of three to five with unlimited access to water and food (chow diet, number 5058, lab diet; or doxycycline chow diet [600 mg/kg], S4107, Bio-Serv, as described for individual experiments). All mice were on a pure C57BL/6J background. Adn-rtTA and FAT-ATTAC mouse lines were generated in the Scherer laboratory as previously described ${ }^{1,2}$. TRE-Cre (Jax stock no. 006234), Rosa26-loxP-STOP-loxPLacZ (Jax stock no. 003309), Rosa26-loxP-STOP-loxP-mT/mG (Jax stock no. 007676), and CAGEGFP (Jax Stock no. 006567) mouse lines were obtained from the Jackson Laboratories. The Institutional Animal Care and Use Committees of City of Hope, Duarte, have approved all animal experiments.

Metabolic cage studies were performed in the City of Hope Comprehensive Metabolic Phenotyping Core facility. Oxygen consumption and energy expenditure were calculated relative to lean mass. GTT was performed in mice without access to food for $12 \mathrm{~h}$ before administration of $2 \mathrm{~g} / \mathrm{kg}$ body weight glucose by intraperitoneal injection. ITT was performed in mice without access to food for six hours before administering $0.75 \mathrm{U} / \mathrm{kg}$ body weight insulin (Eli Lilly) by intraperitoneal injection. To induce adipocyte apoptosis in FAT-ATTAC mice, AP21087 (Ariad Pharmaceuticals) in 4\% ethanol, 10\% PEG-400, 1.75\% Tween-20 in water were administered by intraperitoneal injection at a dose of $0.2 \mu \mathrm{g} / \mathrm{g}$ body weight every day for 7 days.

\section{$\beta$-gal staining}


Mice were anesthetized and perfused with $0.2 \%$ glutaraldehyde in PBS. Adipose tissue was collected and chopped into small pieces. The tissue pieces were fixed in $0.2 \%$ glutaraldehyde/PBS overnight and followed by washed with rinse buffer (100 mM sodium phosphate, $2 \mathrm{mM} \mathrm{MgCl} 2$, $0.01 \%$ sodium deoxycholate, and $0.02 \% \mathrm{NP}-40$ ) three times. Then the tissue pieces were stained with $1 \mathrm{mg} / \mathrm{mL} \beta$-gal in staining buffer containing $5 \mathrm{mM}$ potassium ferricyanide and $5 \mathrm{mM}$ potassium ferrocyanide for $48 \mathrm{~h}$ at room temperature with mild shaking. After staining, the tissue pieces were moved into $10 \%$ neutral buffered formalin overnight for post-fixation. The fixed tissues were processed with a standard paraffin tissue embedding protocol to produce $7 \mu \mathrm{m}$ sections by the City of Hope Pathology Core facility. The sections were then counterstained with nuclear fast red and imaged under a microscope (Keyence).

\section{Isolation of mouse adipose SVFs and enrichment of APC subpopulations}

gWAT was minced and transferred to a $50 \mathrm{~mL}$ Falcon tube containing $10 \mathrm{~mL}$ digestion buffer (100mM HEPES pH 7.4; $120 \mathrm{mM} \mathrm{NaCl} ; 50 \mathrm{mM} \mathrm{KCl} ; 5 \mathrm{mM}$ Glucose; $1 \mathrm{mM} \mathrm{CaCl}_{2}, 1.5 \% \mathrm{BSA}$, and $1 \mathrm{mg} / \mathrm{mL}$ collagenase D). The tissue was incubated in a $37^{\circ} \mathrm{C}$ shaking water bath for $30 \mathrm{~min}$. Digestion was stopped by adding $35 \mathrm{~mL}$ 2\% FBS in PBS. The solution was passed through 100 $\mu \mathrm{m}$ and then $40 \mu \mathrm{m}$ strainers and centrifuged at $400 \mathrm{~g}$ for $5 \mathrm{~min}$. The supernatant was aspirated, and the SVF pellet was collected.

Lin- SVF and enriched ASC, CP-A, CP-1 were obtained by MACS (Stemcell Technologies, Cat\# 17656). To obtain Lin- SVF, anti-mouse-CD45-PE, anti-mouse-CD31-PE, and anti-mouseTer119-PE (1:200, BioLegend) were used. To enrich the ASC subpopulation, additional antimouse-DPP4/CD26-PE antibody (1:50, BioLegend) was used. To enrich CP-A and CP-1 
subpopulations, the above 4 antibodies plus anti-mouse-CD9-PE (1:200, BioLegend) antibodies were used. To obtain immune cells from gWAT, anti-mouse-CD45-PE was used and isolated by MACS.

\section{Flow cytometry}

For flow cytometry analysis, cells were blocked with anti-mouse-CD16/32 (BioLegend, 1:500) for $10 \mathrm{~min}$, and then stained with anti-mouse-CD45-Pacific Blue (BioLegend, 1:400), anti-mouseCD31-Pacific Blue (BioLegend, 1:400), anti-mouse-Ter119-Pacific Blue (BioLegend, 1:400), anti-mouse-CD140a-APC (BioLegend, 1:200), anti-mouse-DPP4/CD26-PE/Cy7 (BioLegend, 1:100), anti-mouse-CD9-PerCP/Cy5.5 (BioLegend, 1:400), anti-LIFR (Proteintech; 1:200), and anti-rabbit-PE/Cy5.5 (Invitrogen, 1:200) ; anti-human-CD45-PE (BioLegend, 1:400), anti-humanCD31-PE (BioLegend, 1:400), anti-human-CD235a-PE (BioLegend, 1:400), and anti-humanCD140a-APC (BioLegend; 1:100) for individual experiments for $20 \mathrm{~min}$ on ice. After antibodies incubation, the cells were washed once with $2 \% \mathrm{FBS} / \mathrm{PBS}$, and analyzed by BD Fortessa cytometer. The flow plots were analyzed by FlowJo (V10).

For FACS isolation of macrophages from gWAT, total SVF were blocked with anti-mouseCD16/32 (BioLegend, 1:500), and then stained with anti-mouse-CD45-PE (BioLegend, 1:400), anti-mouse-CD11b-APC (BioLegend, 1:200), and anti-mouse-F4/80-Alexa Fluor 488 (BioLegend, 1:200). Macrophages were obtained using BD Aria Fusion.

\section{Isolation of human adipose SVFs}


Peri-pancreatic WAT samples from deceased cadaveric donors were dissected from the pancreas (processe time within $\sim 8-12$ hours). All of the proposed experiments will be immediately performed using the fresh tissue samples, without freezing or any preservation. The sampling and examination of human tissues have been approved by the Ethics Committee of COH. WAT from five donors (male, 44-year-old; male, 21-year-old; female, 42-year-old; male, 24-year-old; male, 57-year-old) were used for scRNA-seq, and WAT from four donors (all males, 39, 56, 57, and 58year-old) were used for flow cytometry analysis. Adipose tissue was minced and transferred to a $50 \mathrm{~mL}$ Falcon tube containing $10 \mathrm{~mL}$ digestion buffer $(1 \times \mathrm{PBS}$, and $10 \mathrm{mg} / \mathrm{mL}$ collagenase $\mathrm{D})$. The tissue was incubated in a $37^{\circ} \mathrm{C}$ shaking water bath for $45 \mathrm{~min}$. Digestion was stopped by adding $35 \mathrm{~mL} \mathrm{2 \%} \mathrm{FBS} \mathrm{in} \mathrm{PBS.} \mathrm{The} \mathrm{solution} \mathrm{was} \mathrm{passed} \mathrm{through} \mathrm{a} 250 \mu \mathrm{m}$ mesh (Spectrum), and then $100 \mu \mathrm{m}$ and $40 \mu \mathrm{m}$ strainers, and centrifuged at $600 \mathrm{~g}$ for $5 \mathrm{~min}$. The supernatant was aspirated, and the SVF pellet was collected. To obtain Lin- SVFs, cells were incubated with anti-humanCD45-PE, anti-human-CD31-PE, and anti-human-CD235a-PE (1:200, BioLegend), and isolated by MACS (Stemcell Technologies, Cat\# 17654). To enrich immune cells, anti-human-CD45-PE (1:200, BioLegend) was used and isolated by MACS.

\section{scRNA-seq}

Lin- SVF and CD45+ immune cells from 2.5-month-old or 12-month-old C57BL/6J male mice gWAT or Lin- SVFs from human peri-pancreatic adipose tissue (Extended Data Supplementary Table 4) were freshly collected and resuspended in PBS containing 0.04\% BSA at a concentration of $800 \sim 1000$ cells $/ \mu 1$. Cell number and viability were measured by trypan blue using a TC20 Automated Cell Counter (BioRad). Single-cell RNA libraries were prepared by the City of Hope Integrative Genomics Core facility according to the Chromium ${ }^{\mathrm{TM}}$ Single Cell 3' Reagent Kits v3 
User Guide (10x Genomics). Approximately 10,000 cells were loaded on a Chromium single cell Controller instrument (10x Genomics) to form single cell gel beads in emulsion (GEMs). After reverse transcriptions, GEMs were harvested and the cDNAs were amplified and cleaned up with the SPRIselect Reagent Kit (Beckman Coulter). The barcoded sequencing libraries were constructed using the Chromium Single-Cell 3' Library Kit (10x Genomics) for enzymatic fragmentation, end-repair, A tailing, adaptor ligation, ligation cleanup, sample index PCR, and PCR cleanup. Libraries were then sequenced with a NovaSeq 6000 instrument (Illumina) with a depth of 50-100 k reads per cell.

\section{scRNA-seq data processing and quality control}

The 10X Genomics Cell Ranger version 3.0.2 single-cell software ${ }^{3}$ was used to perform sample demultiplexing sequencing alignment to mouse genome mm10, filtering, and unique molecular identifier (UMI) counting to generate gene count matrices. Single cells were identified from background noise by filtering on the proportion of mitochondrial reads (threshold: $<10 \%$ ), the number of UMI (thresholds: 700-22,000), and the number of detected genes (thresholds: 200$6,000)$.

\section{Identification of cell clusters}

The Seurat R package version $3.0^{4}$ was used to determine cell clusters by the Louvain algorithm based on similarities in the transcriptome patterns and visualized with t-Distributed Stochastic Neighbor Embedding ( $t-$ SNE). The optimal number of Principal Components used for tSNE/UMAP dimensionality reduction and Louvain clustering was determined using the Jackstraw permutation approach and a grid-search of the parameters. The identities of the cell types were 
resolved by comparing the cell cluster specific marker genes expressed in each cluster in our own dataset, as identified with a Wilcoxon rank sum test, with known cell-type specific markers curated from literature, single cell atlases, and previous studies in the SVF. To be considered in the cell cluster marker analysis, a gene had to be expressed in at least $10 \%$ of the single cells from the cluster of interest and there had to be at least a $0.25 \log$ fold change in the cell cluster of interest than in other cells. A multiple testing was corrected using the Benjamini-Hochberg method to estimate false discovery rate (FDR).

\section{Identification of differentially expressed genes (DEGs) and pathways}

To quantitatively determine which genes were affected by aging, we compared the cell transcriptome of each cell type between age groups using a Wilcoxon rank sum test. To be considered in the analysis, a gene had to be expressed in at least $10 \%$ of the single cells from at least one of the two groups for that cell type and there had to be at least 1.1-fold change in gene expression between the groups. Multiple testing correction was done using the BenjaminiHochberg method to estimate FDR.

For ASC, CP-1 and CP-2 populations shared between both the young and aged mice, the DEGs were identified by comparing the two age groups and then subjected to pathway enrichment analysis. Since CP-A is only present in the aged group, the CP-A marker genes in the aged condition were used as DEGs and subject to pathway enrichment analysis. Pathways from HALLMARK $^{5}$ were assessed for overlap with the DEGs using Fisher's exact test and corrected for multiple testing using the Benjamini-Hochberg method to estimate FDR. 


\section{Single-cell trajectory analysis}

To infer cell lineages of complex communities of heterogeneous cells, pseudo-temporal reconstruction was performed by two methods. The Slingshot R package ${ }^{6}$ was used first to infer multiple branching lineages based on the minimum spanning tree followed by simultaneous principal curves. The Cytotrace $\mathrm{R}$ package ${ }^{7}$ was then used to estimate the developmental potential of ten cell sets (five APC subtypes in "aged" and "young" conditions) based on feature selected gene-count measures.

\section{Cell-cell communication analysis}

Both the Cellphone DB database ${ }^{8}$ and the iTALK database ${ }^{9}$ were used to curate ligand-receptor pairs that may mediate intercellular cross-talk between the APC subtypes and immune cell types. Pathway enrichment of the ligand-receptor pairs between cell types were further analyzed based on the overlap with selected pathways from $\mathrm{KEGG}^{10}$, Reactome ${ }^{11}$, BIOCARTA ${ }^{12}$, and HALLMARK $^{5}$. Top ligand-receptors were ranked based on the formula: the expression value of the ligand gene from the ligand-secreting cell type $\mathrm{X}$ the expression value of the receptor gene from the receptor cell type.

\section{In vivo transplantation of APCs and APC subpopulations}

Lin- SVF from the epididymal white adipose tissue (gWAT) of 12-month-old aged Rosa26loxP-STOP-loxP-mT/mG and 2.5-month-old young CAG-GFP mice were isolated and mixed together at a 1:1 ratio. The cells were resuspended in Matrigel (Corning). A total volume of $20 \mu 1$ Matrigel was injected into the subcutaneous adipose depot of recipient mice at a concentration of 10,000 cells $/ \mu 1$. After four weeks, the recipient mice were euthanized, and sWAT was collected. 
The Matrigel containing transplant was dissected under a fluorescence microscope (Keyence), and the whole transplant was captured under a confocal microscope (Zeiss, LSM880).

For single Tomato + SVF transplant, Lin- SVF from the epididymal white adipose tissue (gWAT) of 12-month-old aged and 2.5-month-old young Rosa26-loxP-STOP-loxP-mT/mG mice were isolated. The cells were resuspended in Matrigel (Corning). A total volume of $20 \mu 1$ Matrigel was injected to the subcutaneous adipose depot of recipient mice at a concentration of 5,000 cells $/ \mu 1$. The comparable aged and young Lin- SVF were injected to either side of the subcutaneous adipose depot of the same recipient. After four weeks, the recipient mice were euthanized, and subcutaneous adipose tissue was collected.

For transplantation of ASC, CP-1, and CP-A, each cell population was isolated from Rosa26loxP-STOP-loxP-mT/mG and resuspended in Matrigel at a concentration of 1,000 cells/ $\mu 1$. Then $20 \mu 1$ Matrigel of ASC from 12-month-old aged and 2.5-month-old young mice, or enriched CPA from aged mice and CP-1 from young mice were injected into either side of sWATs of recipient mice. After two weeks, the recipient mice were euthanized, and subcutaneous adipose tissue was collected.

For 3D imaging and quantification of Matrigel transplants, The Matrigel containing transplant was dissected under a fluorescence microscope (Keyence), and the whole transplant was captured under a confocal microscope (Zeiss, LSM880). Original files were imported to Imaris (Bitplane). All the images and videos were made using Imaris. Quantification of mature adipocyte number 
and volume were analyzed by the spot function of Imaris. The function parameters were kept the same between different samples.

\section{In vitro 3D differentiation and differentiation of APCs and APC subpopulations}

Lin- SVF or enriched ASC, CP-1, and CP-A populations were isolated from gWAT of Rosa26loxP-STOP-loxP-mT/mG mice that were 12-month-old or 2.5-month-old. The cells were cultured as described previously ${ }^{13}$. After isolation by MACS, the cells were resuspended in complete SVF culture medium (DMEM/F12, Life technologies 10565018) containing 10\% FBS (Sigma, 16H328), 1\% penicillin/streptomycin, (Hyclone SV30010), and 0.1\% gentamicin (Life technologies 1855724) at a concentration of 2,000 cells/ $\mu 1$. The same volume of Matrigel was added to the suspension, and $10 \mu 1$ mixture was added to the 12 -well plate. The plate was incubated in the $37^{\circ} \mathrm{C} \mathrm{CO}_{2}$ incubator for $15 \mathrm{~min}$ to induce the formation of the gel. Then complete SVF culture medium was added to the wells, and the cells were cultured in the $37^{\circ} \mathrm{C} \mathrm{CO}_{2}$ incubator. After three days or 16 hours, an adipogenic cocktail containing dexamethasone $(1 \mu \mathrm{M})$, insulin $(5$ $\mu \mathrm{g} / \mathrm{ml})$, isobutylmethylxanthine $(0.5 \mathrm{mM})$, and rosiglitazone $(1 \mu \mathrm{M})$ was added to induce adipocyte differentiation. After six days of adipogenic induction, the cells were then stained with Bodipy (Invitrogen) and imaged under a confocal microscope (Zeiss, LSM880). The images were analyzed using Zen blue (Zeiss).

\section{In vitro 3D macrophage and $\mathrm{APC}$ co-culture}

Lin- SVF were isolated from gWAT of Rosa26-loxP-STOP-loxP-mT/mG mice that were 12month-old or 2.5-month-old. Macrophages were isolated from gWAT of C57BL/6 mice that were 12-month-old or 2.5 month-old by FACS. "Aged" Lin- SVF and "aged" macrophages, "aged" Lin- 
SVF and "young" macrophages, "young" Lin- SVF and "aged" macrophage, and "young" LinSVF and "young" macrophages were mixed at 1:1 ratio in the complete SVF medium at a concentration of 4,000 cells $/ \mu 1$. The same volume of Matrigel was added to the suspension, and 10 $\mu 1$ mixture was added to the 12 -well plate. The plate was incubated in the $37^{\circ} \mathrm{C} \mathrm{CO}_{2}$ incubator for 15 min to induce the formation of the gel. Then complete SVF culture medium was added to the wells, and the cells were cultured in the $37^{\circ} \mathrm{C} \mathrm{CO}_{2}$ incubator. After 16 hours, the adipogenic cocktail was added. Adipocytes were stained with Bodipy (Invitrogen) 6 days after adipogenic induction. Images were captured under a confocal microscope (Zeiss, LSM880), and analyzed using Zen blue (Zeiss).

\section{Histological analysis}

$10 \%$ formalin-fixed tissues were processed with a standard paraffin tissue embedding protocol to produce sections for H\&E staining by the City of Hope Pathology Core facility. For immunofluorescence staining, the sections were dewaxed in xylene and rehydrated in a graded series of ethanol to $\mathrm{ddH}_{2} \mathrm{O}$. Slides were placed in $10 \mathrm{mM}$ sodium citrate buffer and boiled for 30 min for antigen retrieval, then blocked in PBST with 5\% BSA for 30 min. Primary antibodies used were LIFR (1:200, Proteintech), PDGFR $\alpha$ (1:400, R\&D), Perilipin (1:500, Novus), F4/80 (1:400, Abcam), GFP (1:400, Abcam), and mCherry (1:100, Abcam) as described for individual experiments, and incubated at $4^{\circ} \mathrm{C}$ overnight. After three times wash with PBS, secondary antibodies used were Alexa Fluor 647 Donkey-anti-Rabbit IgG H\&L (1:200, Invitrogen), Alexa Fluor 488 Donkey-anti-Goat IgG H\&L (1:200, Invitrogen), Alexa Fluor 594 Donkey-anti-Rat IgG H\&L (1:200, Invitrogen), and Cy3 Donkey-anti-Chicken IgG H\&L (1:200, Jackson Immuno, Inc.), and incubated at room temperature for 2 hours. Slides were then counterstained with DAPI and 
mounted with prolong Anti-Fade mounting medium (Invitrogen) after being washed with PBS three times. Images were acquired using a fluorescence microscope (Keyence).

\section{Statistical analysis}

Graphs are generated with GraphPad Prism. Statistical analysis was also performed within GraphPad Prism. Data values are presented as means \pm s.e.m.. The statistical parameters (i.e. $n$ number, $\mathrm{p}$ values, and numbers of biological repeats) can be found in Figure Legends.

\section{Reference for method}

1 Sun, K. et al. Dichotomous effects of VEGF-A on adipose tissue dysfunction. Proc Natl Acad Sci U S A 109, 5874-5879, doi:10.1073/pnas.1200447109 (2012).

2 Pajvani, U. B. et al. Fat apoptosis through targeted activation of caspase 8: a new mouse model of inducible and reversible lipoatrophy. Nat Med 11, 797-803, doi:10.1038/nm1262 (2005).

3 Zheng, G. X. Y. et al. Massively parallel digital transcriptional profiling of single cells. Nature Communications 8, 14049, doi:10.1038/ncomms14049 (2017).

4 Stuart, T. et al. Comprehensive Integration of Single-Cell Data. Cell 177, 18881902.e1821, doi:10.1016/j.cell.2019.05.031 (2019).

5 Liberzon, A. et al. The Molecular Signatures Database (MSigDB) hallmark gene set collection. Cell Syst 1, 417-425, doi:10.1016/j.cels.2015.12.004 (2015).

6 Street, K. et al. Slingshot: cell lineage and pseudotime inference for single-cell transcriptomics. BMC Genomics 19, 477, doi:10.1186/s12864-018-4772-0 (2018). 
7 Gulati, G. S. et al. Single-cell transcriptional diversity is a hallmark of developmental potential. Science 367, 405-411, doi:10.1126/science.aax0249 (2020).

8 Efremova, M., Vento-Tormo, M., Teichmann, S. A. \& Vento-Tormo, R. CellPhoneDB: inferring cell-cell communication from combined expression of multi-subunit ligandreceptor complexes. Nat Protoc 15, 1484-1506, doi:10.1038/s41596-020-0292-x (2020).

9 Wang, Y. et al. iTALK: an R Package to Characterize and Illustrate Intercellular Communication. bioRxiv, 507871, doi:10.1101/507871 (2019).

10 Kanehisa, M. \& Goto, S. KEGG: kyoto encyclopedia of genes and genomes. Nucleic Acids Res 28, 27-30, doi:10.1093/nar/28.1.27 (2000).

11 Jassal, B. et al. The reactome pathway knowledgebase. Nucleic Acids Res 48, D498d503, doi:10.1093/nar/gkz1031 (2020).

12 BioCarta. Biotech Software \& Internet Report 2, 117-120, doi:10.1089/152791601750294344 (2001).

13 Emont, M. P. et al. Using a 3D Culture System to Differentiate Visceral Adipocytes In Vitro. Endocrinology 156, 4761-4768, doi:10.1210/en.2015-1567 (2015). 


\section{Figure legend}

735

736

737

738

739

740

741

742

743

744

745

746

747

748

749

750

751

752

753

754

755

756

\section{Fig. 1: Aged mice display substantial fat gain and reduced metabolic rate, accompanied by} massive adipogenesis.

a, b, Images (a) and body weight (b) of young (2.5-month-old) and aged (12-month-old) male mice ( $\mathrm{n}=8$ mice per age group).

c, d, Magnetic resonance imaging (MRI) was used to measure fat mass (c) and lean mass (d) (n=8 mice per age group).

e, f, Tissue weight and whole-tissue pictures of gWAT (e) and sWAT (f). $n=9$ mice per each age group.

g-i, Metabolic cage determines oxygen consumption (g), physical activity (h), and energy expenditure (i) in young and aged mice. $n=8$ mice per age group.

$\mathbf{j}-\mathbf{m}, \operatorname{GTT}(\mathrm{j}, \mathrm{k})$ and ITT $(1, \mathrm{~m})$ were performed on young (2.5-month-old) and aged (12-monthold) mice. For GTT, n=8 per age group; for ITT, $n=7$, young; $n=8$, aged.

$\mathbf{n}$, Representative $\beta$-gal staining of mouse gWAT and sWAT samples from the male mice at the indicated ages. Red dashed line circles clusters of new, LacZ-negative adipocytes. These images are representative of three independent experiments.

b-f, k, m, ${ }^{* *} \mathrm{p}<0.01$; two-tailed Student's t-test. j, 1, ${ }^{*} \mathrm{p}<0.05 ;{ }^{*} \mathrm{p}<0.01$; Two-way ANOVA. All data represent the mean \pm s.e.m.

\section{Fig. 2: APCs in aged mice have a high adipogenic rate in vivo.}

a, APCs (Lin-, CD45-CD31-Ter119-) were enriched from isolated SVFs from both 2.5-month-old (young) male CAG-EGFP and 12-month-old (aged) Rosa26-mT/mG mice. An equal number of GFP + and Tomato + cells $\left(1 \times 10^{5}\right)$ were mixed together and suspended in Matrigel. The cell mixture 
757 was transplanted into the sWAT of 2.5-month-old male WT mice.

758 b, Dissected Matrigel plugs were profiled for 3-dimensional Tomato and GFP signals.

759 c, d, Quantification of Tomato + and GFP+ adipocyte number (c) and volume (d). n=3 760 transplantations.

761 e, Hematoxylin and eosin (HE) staining in the transplanted Matrigel plug within the sWAT.

762 f, Immunofluorescence staining of GFP, Tomato, and Perilipin in the transplanted Matrigel plug.

763 g, APCs (Lin-, CD45-CD31-Ter119-) were enriched from isolated SVFs from both 2.5-month-old

764 (young) or 12-month-old (aged) Rosa26-mT/mG mice. These Tomato + cells $\left(1 \times 10^{5}\right)$ were

765 suspended in Matrigel. The cell mixture was transplanted into either side of the sWATof the same

766 2.5-month-old male WT mice.

767 h, Dissected Matrigel plugs were profiled for 3-dimensional Tomato signal.

$768 \mathbf{i}, \mathbf{j}$, Quantification of Tomato+ adipocyte number (i) and volume (j). $\mathrm{n}=4$ mice (transplants).

$769 \mathrm{c}, \mathrm{d}, \mathrm{i}, \mathrm{j},{ }^{*} \mathrm{p}<0.05 ;{ }^{* *} \mathrm{p}<0.01$; two-tailed Student's t-test. All data represent the mean \pm s.e.m.

770

771 Fig. 3: ScRNA-seq analysis identifies a novel, age-specific APC population.

772 a, Progenitor populations from each group (4,337 "young" and 10,757 "aged" cells) were selected

773 for t-distributed stochastic neighbor embedding (tSNE) plot. K-means clustering $(\mathrm{k}=25)$ was

774 applied to the tSNE plot to identify five clusters, ASC, IAP, CP-1, CP-2, and CP-A. This data is 775 from a single experiment. $\mathrm{n}=3$ per group.

776 b, $\mathbf{c}$, tSNE plot showing differences between young and aged adipocyte APCs, indicating the CP-

777 A cluster uniquely exists in the aged group.

778 d, Slingshot trajectory analysis of five clusters showing a new, age-specific trajectory from ASC 779 to CP-A. 
e, The percentage of each cluster in young and aged APCs. $n=3$ per group. ${ }^{*} \mathrm{p}<0.01$; Two-way ANOVA. All data represent the mean \pm s.e.m.

f, Box plot showing median and distribution of CytoTRACE values (modified gene count measure) per APC subtypes in young and aged mice. A Higher CytoTRACE value means less differentiated 784 status.

$785 \mathbf{g}-\mathbf{k}$, Individual gene tSNE and violin plots showing the expression and distribution of 786 representative marker genes: general APC marker Pdgfra (g), cluster-specific markers $C d 55$ for 787 ASC (h), Apoe for CP-1 (i), Mfap4 for CP2 (j), and Thbs1 for CP-A (k).

788 1, Expression levels of genes related to adipogenesis in the five APC populations.

$789 \mathbf{m}$, The grogenitor populations from 5 human donors were selected for tSNE plot.

$790 \mathbf{n}$, ASC and CP-A score of clusters in human APCs. The scores calculate the top 20 ASC and CP791 A marker genes for each cell.

792 o, Flow cytometry analysis shows the percentage of LIFR+ populations within the PDGFR $\alpha+$ 793 population in each peripancreatic WAT sample from men of different ages.

795 Fig. 4: CP-A cells are highly proliferative and adipogenic in vitro and in vivo.

796 a, Top upregulated pathways in "aged" ASCs compared to "young" ASCs.

797 b, Top pathways in the age-specific CP-A population.

798 Enrichment score ( $\mathrm{a}$ and $\mathrm{b}$ ): Number of Overlapped genes/number of genes in our cell type specific 799 geneset $\times 20000 /$ number of genes in the pathway. Pathway source: HALLMARK. Statistic cut off: $800 \quad \mathrm{FDR}<0.05$

801 c, Young ASC and CP-1 populations were enriched from the SVFs isolated from the gWAT of 802 2.5-month-old (young) male Rosa26-mT/mG mice; aged ASC and CP-A populations were 
803

804

805

806

807

808

809

810

811 812 (aged).

813

814

815

816

817

818

819

820

821

823

824

825

$822 \mathbf{k}$, Quantification of Tomato+ adipocyte number and volume generated from young and aged

enriched from the SVFs isolated from the gWAT of 12-month-old (aged) male Rosa26-mT/mG mice. For each APC population, $1 \times 10^{4}$ cells were suspended in Matrigel and seeded in tissue culture dishes. 16 hours later, the adipogenic cocktail was added to induce APC differentiation. Cells were imaged 6 days later with BODIPY labeling lipid droplets.

d, f, Representative confocal fluorescent images show Tomato + cells (all cells seeded) and GFP+ cells (adipocytes are positive for BODIPY staining) from the young and aged ASC populations (d), young CP-1 poulation and aged CP-A population (f).

e, g, Quantification of adipogenesis rate, adipocyte number (GFP+ cells), and total cell number (Tomato + cells). e, $n=3$ transplantations; $g, n=3$ transplantations (young) $n=4$ transplantations

h, Young DPP4- (none ASC APCs) and CP-1 populations were enriched from the SVFs isolated from the gWAT of 2.5-month-old (young) male Rosa26-mT/mG mice. Aged DPP4- (none ASC APCs) and CP-A populations were enriched from the SVFs isolated from the gWAT of 12-monthold (aged) male Rosa26-mT/mG mice. For each APC population, $2 \times 10^{4}$ cells were enriched. Each enriched APC population was suspended in Matrigel and transplanted into the sWAT of 2.5month-old WT male mice. Young and aged DPP4- populations were transplanted into either side of the sWATof the same 2.5-month-old male WT mice. Young CP-1 and aged CP-A populations were transplanted into either side of the sWATof the same 2.5-month-old male WT mice.

i, j, l, m, Dissected Matrigel plugs were profiled for 3-dimensional Tomato signals. 23 DPP4- cells. $\mathrm{n}=3$ transplantations.

24 n, Quantification of Tomato+ adipocyte number and volume generated from young CP-1 and Aged CP-A cells. $n=4$ transplantations. 
${ }^{*} \mathrm{p}<0.05 ;{ }^{*} \mathrm{p}<0.01 ;$ two-tailed Student's t-test. All data represent the mean \pm s.e.m. 
Figure 1
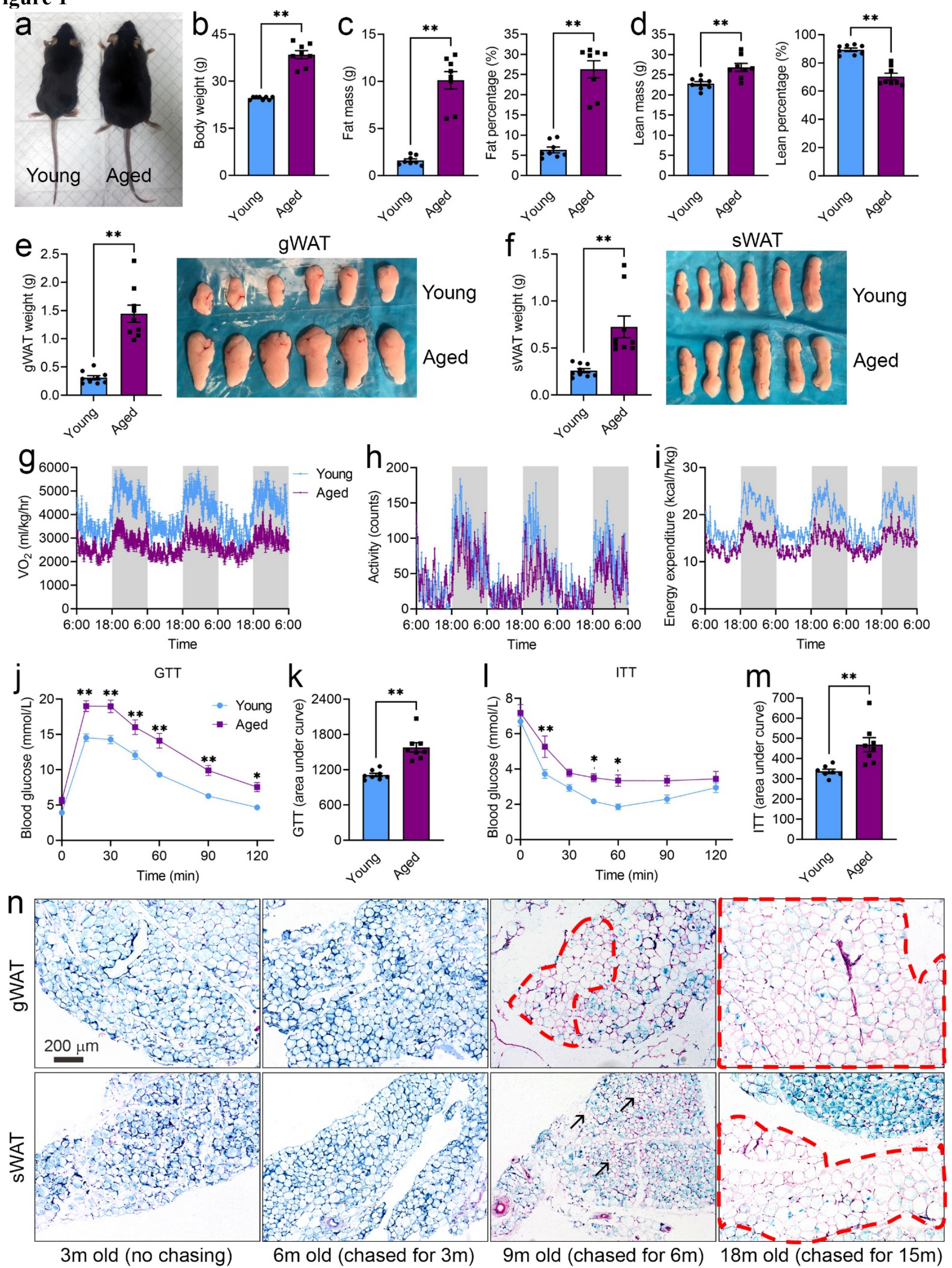
Figure 2
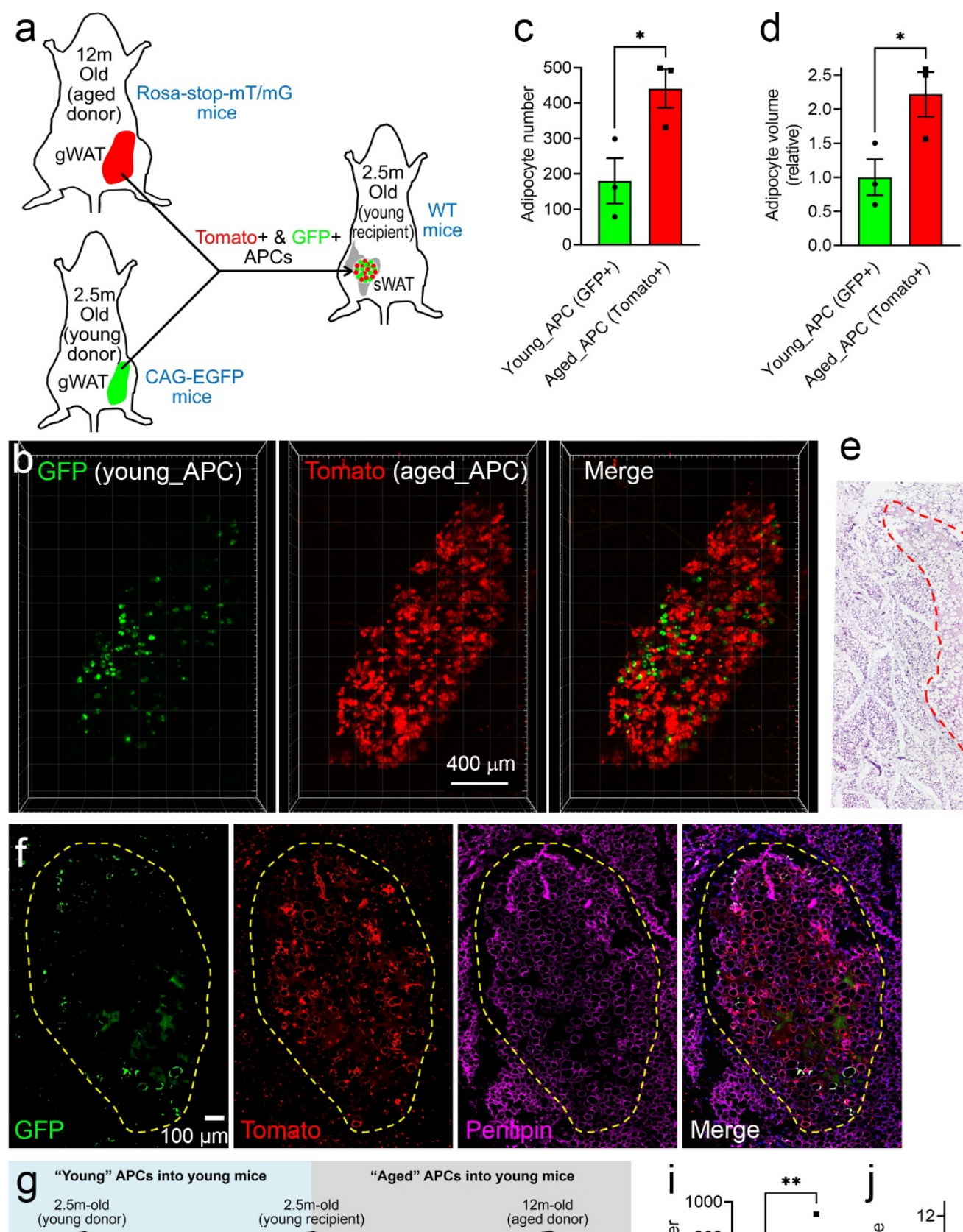

e
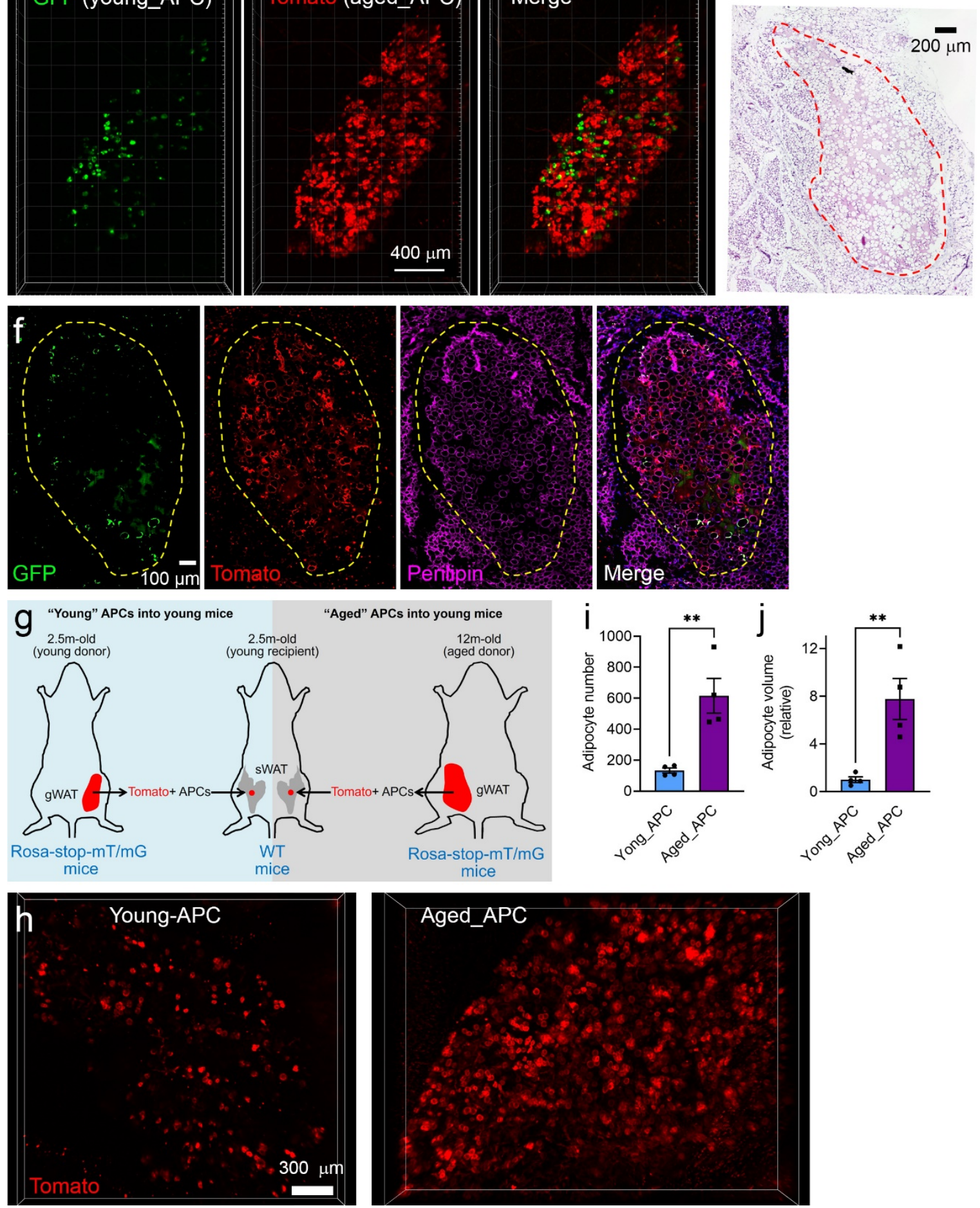
Figure 3
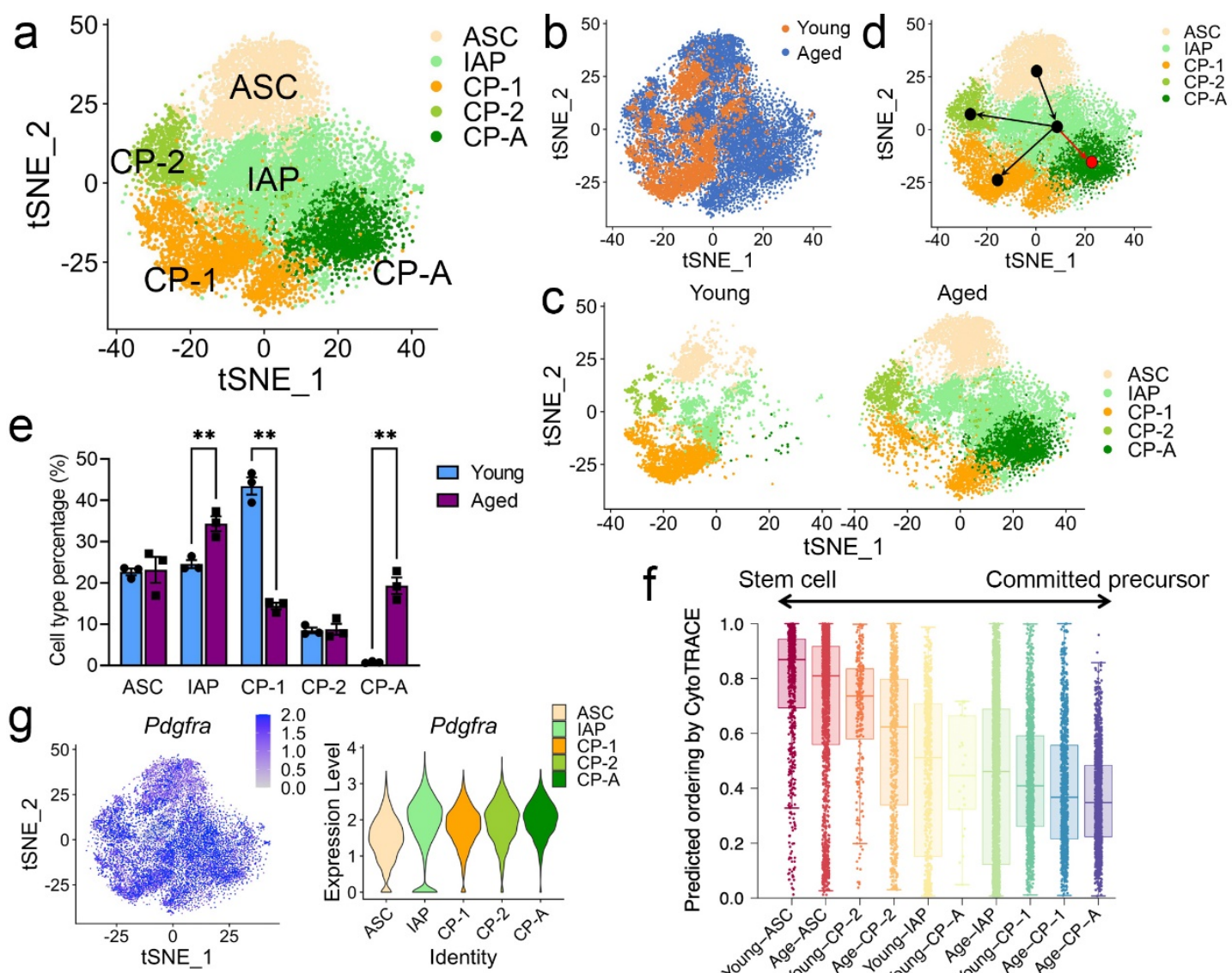

Aged
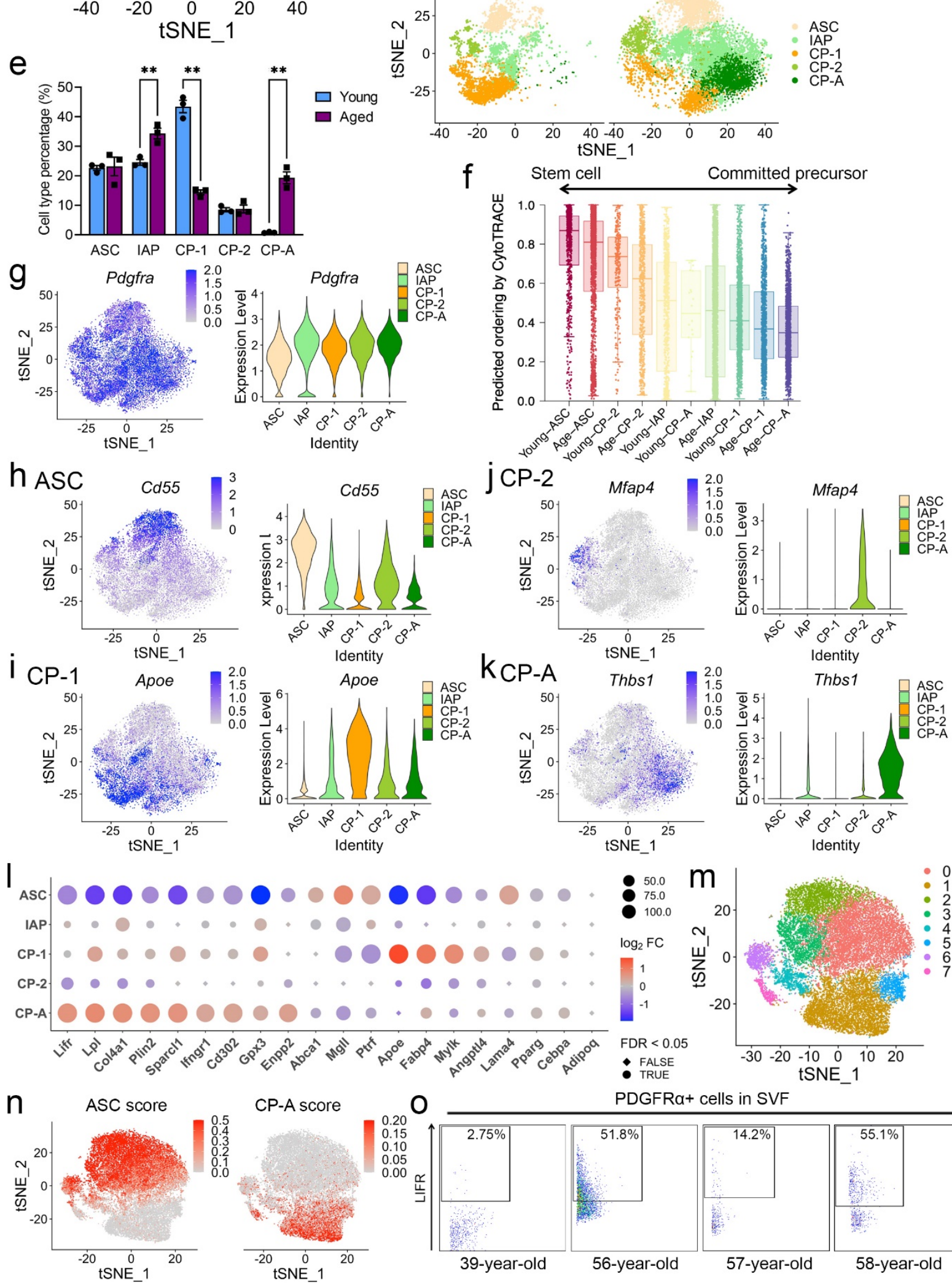

0

PDGFRa+ cells in SVF

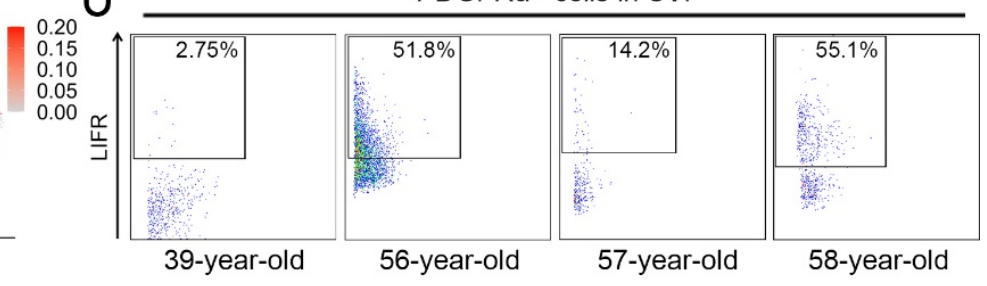




\section{Figure 4}

a Upregulated pathways in aged ASC $\mathbf{b}$

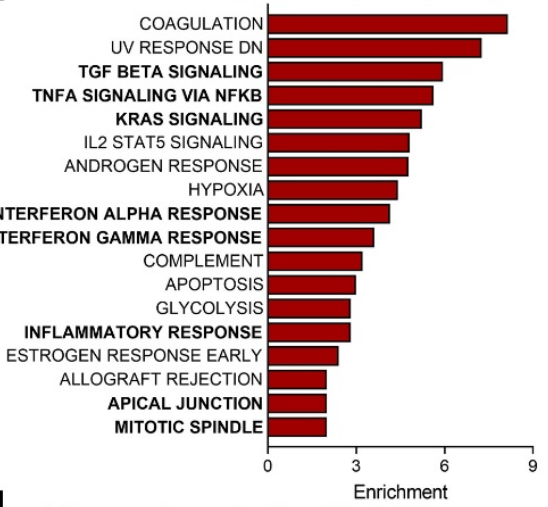

d Adipogenic cocktail added $16 \mathrm{~h}$ post seeding

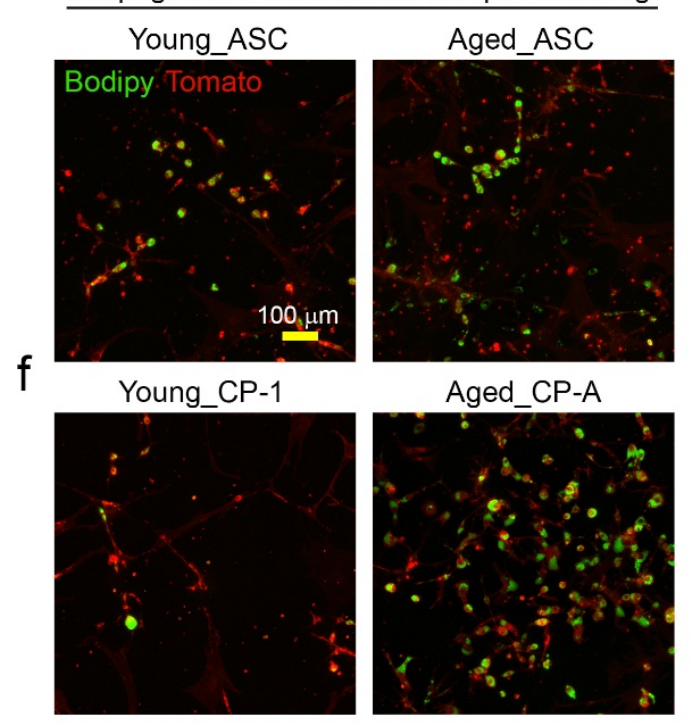

h
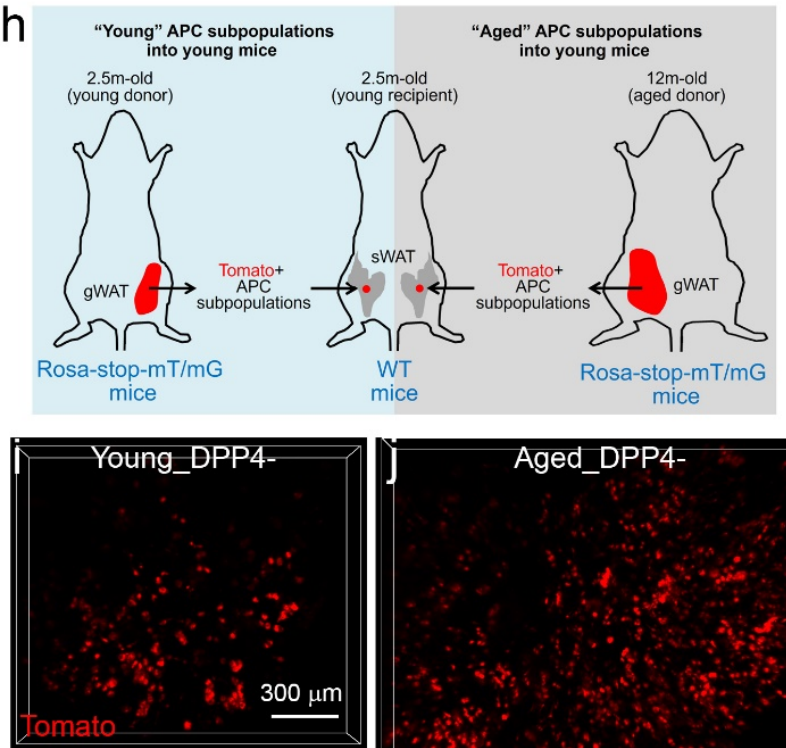

e
Top pathways in aged ASC C
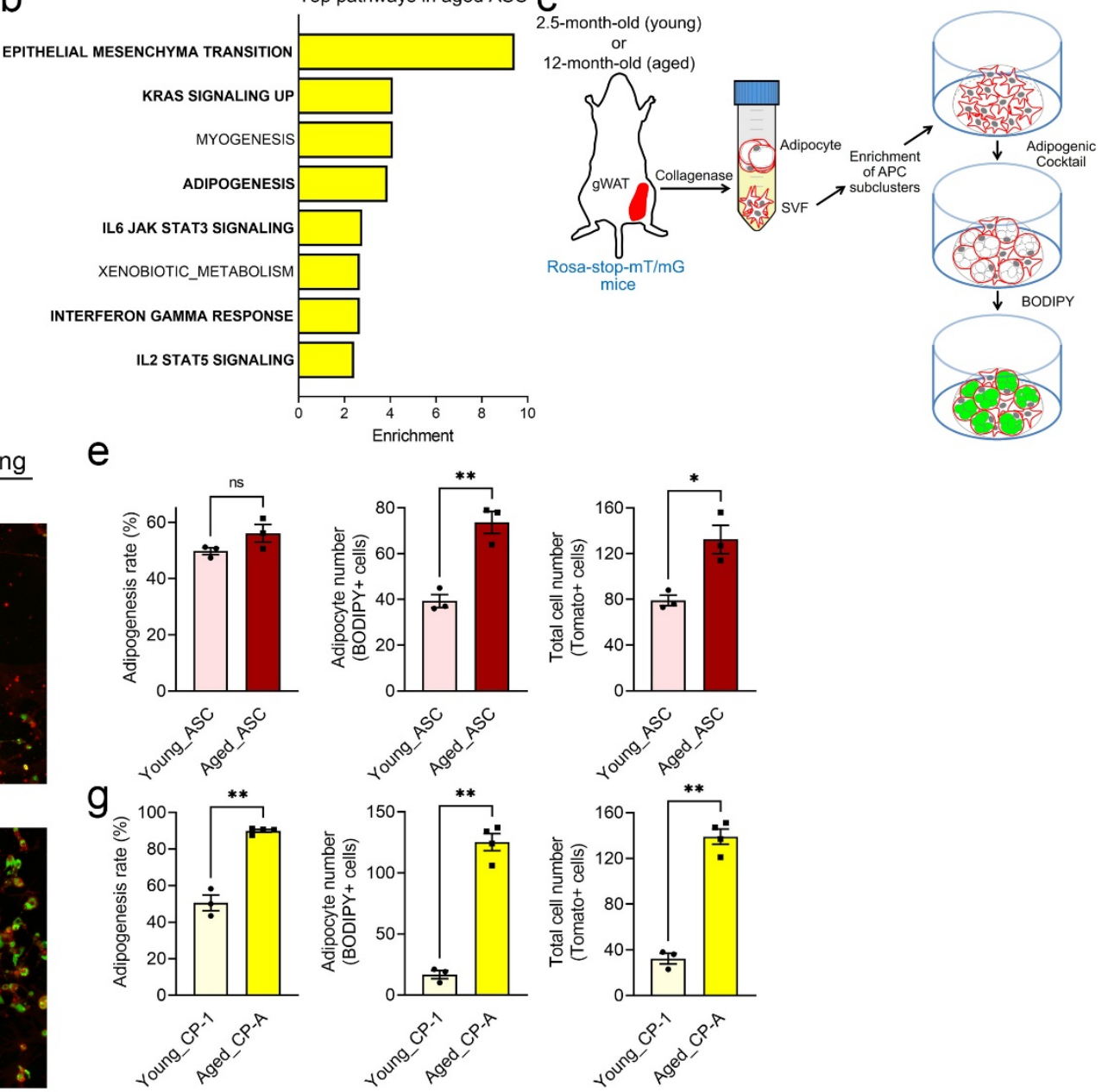

$\mathrm{k}$
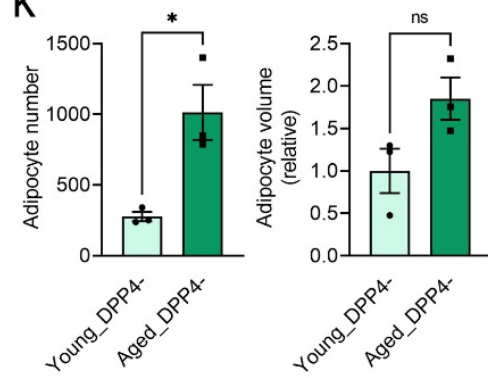

$\mathrm{n}$

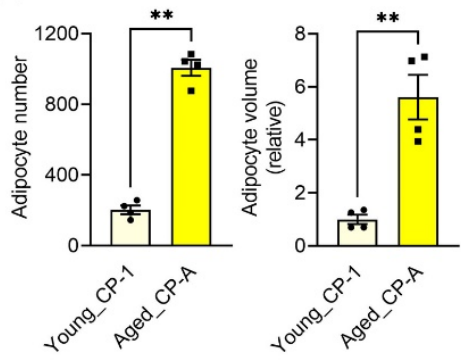

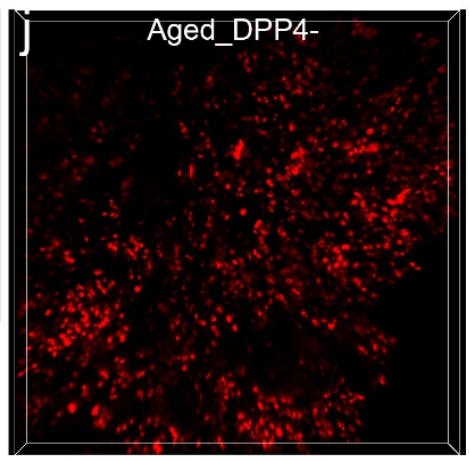
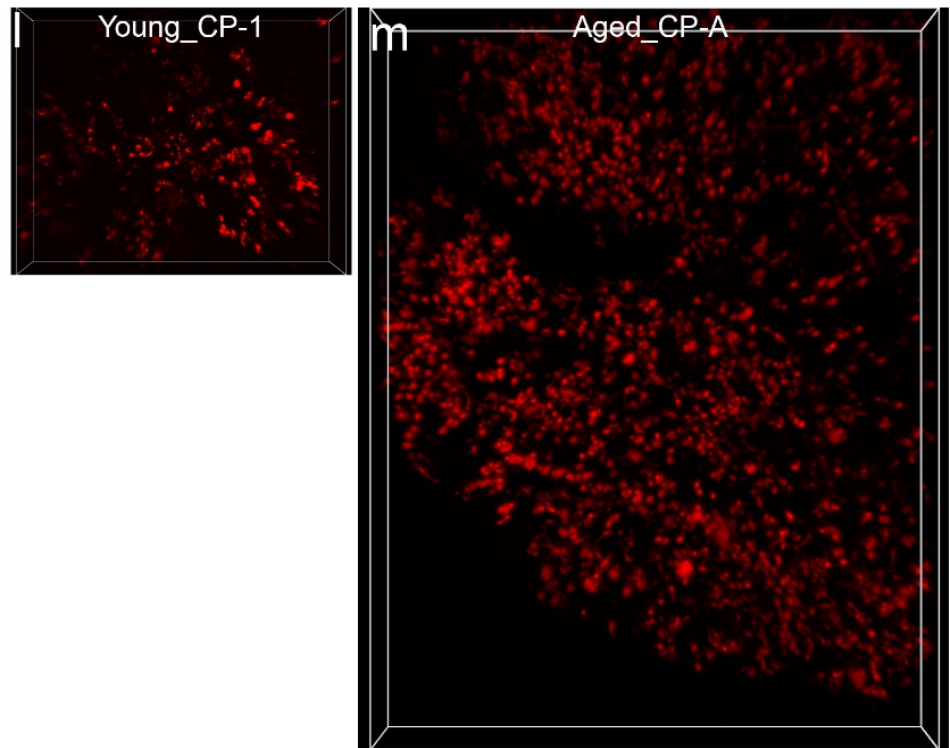


\section{Extended data figure legend}

Extended Data Fig. 1: In vivo tracking of adipogenesis shows sex difference in age-related adiposity and adipogenesis

a, Magnetic resonance imaging (MRI) was used to measure water content ( $n=8$ mice per age group).

b, c, Bodyweight curve (b) and body weight gain (c) of male and female C57BL/6J mice from 8week-old to 44 -week-old ( $n=9$ male mice; $n=8$ female mice).

d, e, Metabolic cage studies show total distance (d), and food intake (e) in young and aged mice. $\mathrm{n}=8$ mice per age group.

f, Doxycycline inducible, adipocyte-specific labeling with LacZ in the AdipoChaser-LacZ mouse model. The doxycycline (dox)-based, tet-responsive, Cre-loxP "pulse-chase" labeling system is derived from interbreeding three transgenic strains: 1) mice expressing the "tet-on" transcription factor rtTA under the adiponectin promoter (Adn-rtTA); 2) mice expressing tet-responsive CRE (TRE-Cre) that is activated by rtTA in the presence of doxycycline; and 3) reporter mice expressing a LacZ reporter gene from the Rosa26 locus in a Cre-dependent manner (Rosa26-loxpstop-loxP-LacZ). This model allows tracking the fate of pre-existing adipocytes (LacZ+) and the identification of newly generated adipocytes (LacZ-negative) in mice exposed to doxycycline.

g, 3-month-old male and female AdipoChaser-LacZ mice were fed with a doxycycline diet for 1 week to ensure a uniform and permanent labeling of all mature adipocytes, followed by a standard chow diet for 3 to 15 months.

$\mathbf{h}$, Representative $\beta$-gal staining of mouse gWAT and sWAT samples from the female mice at the indicated ages. Arrows indicate new, LacZ-negative adipocytes. These images are representative of three independent experiments. 
a, c-e, ${ }^{*} \mathrm{p}<0.05 ;{ }^{* *} \mathrm{p}<0.01$; two-tailed Student's t-test. All data represent the mean \pm s.e.m.

Extended Data Fig. 2: Aged mice have higher WAT regeneration rate.

a, Experimental design: young or aged FAT-ATTAC mice were dimerized daily for seven days, and gWAT samples were collected before, or 0, 1, 2, 4 weeks after dimerization.

b, Hematoxylin and eosin (HE) staining in the gWAT of young (2.5-month-old) and aged (12month-old) FAT-ATTAC mice before and after dimerization. These images are representative of two independent experiments.

c, Perilipin (green), F4/80 (red), and DAPI (blue) immunofluorescence staining in gWAT of young (2.5-month-old) and aged (12-month-old) FAT-ATTAC mice after dimerization. These images are representative of two independent experiments.

d, Whole-tissue images of gWAT from young (2.5-month-old) and aged (12-month-old) FATATTAC mice after dimerization.

Extended Data Fig. 3: Aged microenvironment does not promote adipogenesis of APCs in young mice.

a, Lin- (CD45-CD31-Ter119-) SVFs were isolated from 2.5-month-old (young) male Rosa26$\mathrm{mT} / \mathrm{mG}$ mice. $1 \times 10^{5}$ cells were suspended in Matrigel and transplanted into the sWAT of 2.5month-old (young) or 12-month-old (aged) male WT mice.

b, Dissected Matrigel plugs were profiled for 3-dimensional Tomato signal.

c, Similar experiments were performed using Lin- (CD45-CD31-Ter119-) SVFs isolated from 2.5month-old (young) male CAG-EGFP mice.

d, Dissected Matrigel plugs were profiled for 3-dimensional GFP signal. 


\section{Extended Data Fig. 4: scRNA-seq of Lin-SVFs in gWAT of young and aged male mice.}

a, Lin- (CD45-CD31-Ter119-) SVFs isolated from gWAT of 2.5-month-old (young) and 12month-old (age) male WT mice were used for scRNA-seq.

b, Three major cell clusters, progenitor cells, immune cells, and endothelial cells were identified using k-means $=25$.

c, Lin- SVFs from each mouse (2,199 cells from "young 1", 2,394 cells from "young 2", 2,539 cells from "young 3", 5,150 cells from "aged 1", 4,109 cells from "aged 2", and 3,072 cells from "aged 3") are illustrated in the tSNE plot.

d, tSNE plot showing differences between young and aged Lin- SVFs.

e, Slingshot trajectory analysis of five APC clusters in each age group, showing a new, age-specific trajectory from ASC to CP-A in the aged group.

f, Barplot shows the top 10 (less differentiated; red) and bottom 10 (most differentiated; blue) genes in this dataset based on their correlation with CytoTRACE values.

g, Box plot showing the median and distribution of CytoTRACE values (modified gene count measure) per APC population in young and aged mice, separately.

h, Dot plot showing the scaled average expression of the top three marker genes in each APC cluster (young and aged combined).

$\mathbf{i}, \mathbf{j}$, Individual gene tSNE and violin plots, showing the expression and distribution of the ASC marker Dpp4 (i), and the CP-A marker Lifr (j).

Extended Data Fig. 5: Comparison of ACS, IAP, CP-1, CP-2, and CP-A populations with previously identified APC populations. 
a, tSNE plot showing the top 50 markers of ASC1 and ASC2 as reported by Burl et al. ${ }^{15}$ overlaid on the scRNA-seq analysis of eWAT from 2.5-month-old (young) or 12-month-old (aged) male WT mice.

b, Group identities with the 50 top cluster markers as reported by Burl et al. ${ }^{15}$.

c, tSNE plot showing the top 50 markers of FAP1-4 as reported by Sárvári et al. ${ }^{20}$ overlaid on the scRNA-seq analysis of eWAT from 2.5-month-old (young) or 12-month-old (aged) male WT mice. d, Group identities with the 50 top cluster markers as reported by Sárvári et al. ${ }^{20}$.

e, tSNE plot showing the top 50 markers of P1-3 as reported by Schwalie et al. ${ }^{17}$ overlaid on the scRNA-seq analysis of sWAT from 2.5-month-old (young) or 12-month-old (aged) male WT mice. f, Group identities with the 50 top cluster markers as reported by Schwalie et al. ${ }^{17}$.

\section{Extended Data Fig. 6: Single-cell RNA-seq analysis of human APCs}

a, Four major cell clusters, progenitor cells, immune cells, mesothelial cells and endothelial cells were identified with k-means clustering (k-means=25) applied to the tSNE plot.

b-i, Individual gene tSNE plots showing the expression levels of the indicated genes: adipocyte stem cell/progenitor markers Pdgfra (b) and Pdgfrb (c), ASC markers Cd55 (d), and Pi16 (e), CPA marker Lifr (f), and committed preadipocyte markers Pparg (g), Fabp4 (h), and Lpl (i).

j, Flow cytometry strategy for identifying PDGFR $\alpha+$ cells. Single cells were selected based on forward and side scatter profiles. Live cells were gated as DAPI-. Immune cells, endothelial cells, and erythrocytes were gated out using CD45, CD31, and CD235a antibodies.

\section{Extended Data Fig. 7: Enriching APC populations and in vitro 3D culture of APCs.}

a, Representative confocal fluorescent images show young and aged Lin- (CD45-CD31-Ter119-) 
total APCs (all Tomato + cells that seeded) and GFP + cells (BODIPY+, adipocytes). The adipogenic cocktail was added 3 days post-seeding. Other experimental conditions are the same as described in Fig. 4c. $\mathrm{n}=2$ per group. All data represent the mean \pm s.e.m.

b, Representative confocal fluorescent images show young and aged Lin- (CD45-CD31-Ter119-) total APCs (all Tomato + cells that seeded) and GFP + cells (adipocytes). The adipogenic cocktail was added 16 hours post-seeding. Other experimental conditions are the same as described in Fig. 4c. $\mathrm{n}=3$ per group. ${ }^{*} \mathrm{p}<0.05 ;{ }^{*} \mathrm{p}<0.01$; two-tailed Student's t-test. All data represent the mean \pm s.e.m.

c, SVFs from gWAT of young and aged male WT mice were subjected to flow cytometry analysis. Cells were first enriched for APCs (CD31-CD45-Ter119-PDGFR $\alpha+$ ). Flow cytometry analysis shows the percentage of LIFR+ populations within the PDGFR $\alpha+$ population in $\mathrm{gWAT}$ of 2.5month-old (young) or 12-month-old (aged) male WT mice.

d, DPP4+ population was enriched by magnetic bead-associated cell sorting (MACS). Flow cytometry analysis validates the isolation of DPP4+ and DPP4- populations within the Lin- SVF after MACS in 2.5-month-old (young) or 12-month-old (aged) male WT mice.

e, CP-1 and CP-A populations were enriched through MACS-based negative selection, depleting DPP4+ ASC, CD9+ CP-2, and IAP populations in Lin- SVFs. Flow cytometry analysis validates depletion of DPP4+ and CD9+ populations from the enriched CP-A population after MACS from 2.5-month-old (young) or 12-month-old (aged) male WT mice.

f, Flow cytometry analysis validates increased LIFR + cell percentage in enriched CP-A population after MACS.

g, Flow cytometry analysis shows DPP4+ and CD9+ population percentages in PDGFR $\alpha+$ eWAT SVFs from 2.5-month-old (young) or 12-month-old (aged) male WT mice. 


\section{Extended Data Fig. 8: scRNA-seq of immune cells isolated from the sWAT of young and aged}

mice.

a, CD45+ SVFs from each group (5,528 "young" and 4,986 "aged" cells) were selected for tSNE plot. 15 clusters, including 12 types of immune cell were generated using $\mathrm{k}$-means $=25$. This data is from a single experiment. "MAC" indicates macrophages, "MAC2" indicate M2 macrophages. $\mathrm{n}=3$ per group.

b, Dot plot showing the scaled average expression of representative marker genes in each immune cell clusters (young and aged combined).

c, CD45+ SVFs from each mouse (2,046 "young 1", 1,686 "young 2", 1,796 "young 3", 1,784 "aged 1", 1,130 "aged 2", and 2,072 "aged 3") are illustrated in tSNE plot.

d, tSNE plot showing differences between young and aged immune cells.

e. Flow cytometry analysis shows the percentage of macrophage $(\mathrm{CD} 11 \mathrm{~b}+)$ within the $\mathrm{CD} 45+$ population in 2.5-month-old (young) or 12-month-old (aged) male WT mice, and the percentage of M2 macrophages (CD206+) within the CD11b+ population.

f, Heatmap illustrating the numbers of ligand receptor pairs gained or lost in aged mice comparing to young mice between immune cell types and APC populations. 2D-clustering was applied to order the cell types.

$\mathbf{g}-\mathbf{l}$, One-way ligand-receptor communication analysis of the top immune cell-APC interaction events in selected pathways: adipogenesis $(g, h), \operatorname{TGF} \beta(i, j)$, and insulin $(k, 1)$, in young and aged mice. Circos plots showing top 40 highly expressed ligand-receptor pair signaling with ligand from immune cells to receptors in APC populations. 
Extended Data Fig. 9: Increased proliferation and adipogenesis signaling from macrophage to aged APC populations.

$\mathbf{a}-\mathbf{c}$, One-way ligand-receptor communication analysis for all putative gain or loss of macrophageASC interaction events in aging via selected pathways: adipogenesis (a), TGF $\beta$ (b), and insulin (c). Circos plots showing ligand-receptor pair signaling with ligand from MAC, MAC2 to receptors in ASC.

$\mathbf{d}-\mathbf{i}$, One-way ligand-receptor communication analysis the top young macrophage-young $\mathrm{CP}-1$ and aged macrophage-aged CP-A interaction events in selected pathways: adipogenesis (d, g), TGF $\beta$ (e, h), and insulin (f, i). Circos plots showing top 40 highly expressed ligand-receptor pair signaling with ligand from MAC, MAC2 to receptors in young CP-1 (d-f) and aged CP-A ( $\mathrm{g}-\mathrm{i})$.

\section{Extended Data Fig. 10: Macrophages co-culture increases proliferation of both "young" and “aged" APCs.}

a, Total APCs (Lin-, CD45-CD31-Ter119-) were enriched from 2.5-month-old (young) or 12month-old (aged) male Rosa26-mT/mG mice were enriched. CD45+ immune cells were enriched from 2.5-month-old (young) or 12-month-old (aged) male WT mice. $1 \times 10^{4}$ APCs and $1 \times 10^{4}$ immune Cells were mixed in Matrigel and seeded in tissue culture dishes. 16 hours later, the adipogenic cocktail was added to induce APC differentiation. Cells were imaged 6 days later with BODIPY labeling lipid droplets.

b, Representative confocal fluorescent images show Tomato + cells (all cells seeded) and GFP+ cells (adipocytes) and quantification of adipogenesis rate, adipocyte number (GFP+ cells), and total cell number (Tomato + cells). $\mathrm{n}=2$ per group. All data represent the mean \pm s.e.m.

c, Summarization of key findings: APCs in young adult mice are quiescent. These cells do not 
undergo adipogenesis unless triggered by metabolic challenges (such as high fat diet feeding or cold exposure). The aging process remodels APCs, generates ASC cells that are highly proliferative and $\mathrm{CP}-\mathrm{A}$ cells that are both highly proliferative and adipogenic. The proliferation and differentiation of APCs in aged mice are spontaneous, without any external metabolic challenges. Illustration created with BioRender.com. 


\section{Extended Data Fig. 1}
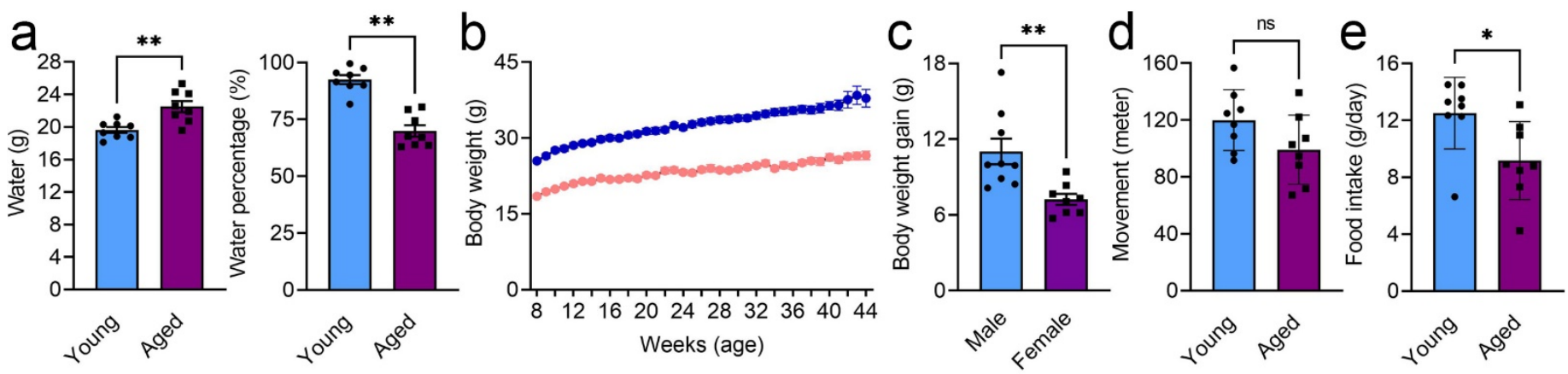

f AdipoChaser-LacZ mouse
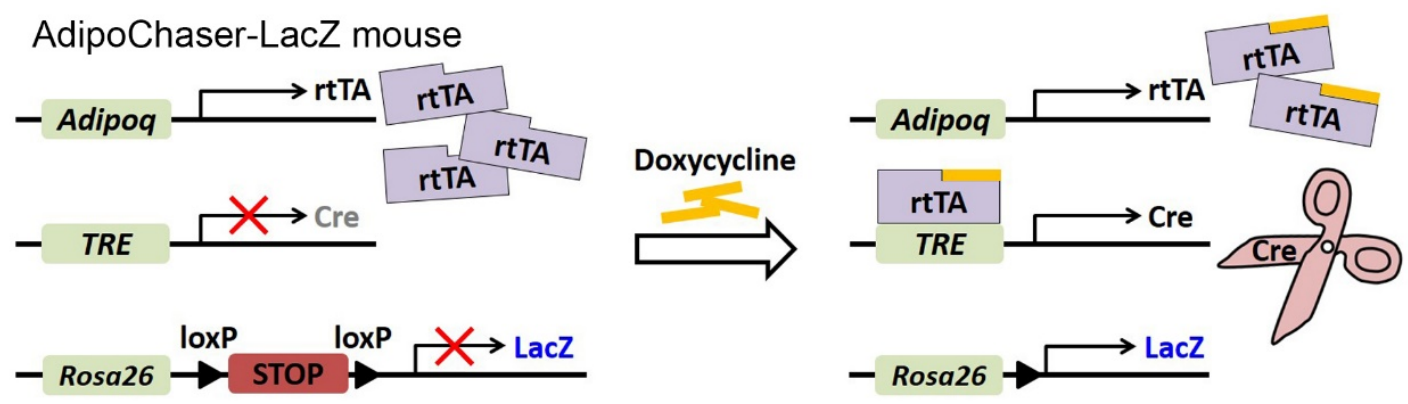

9 AdipoChaser-Lacz
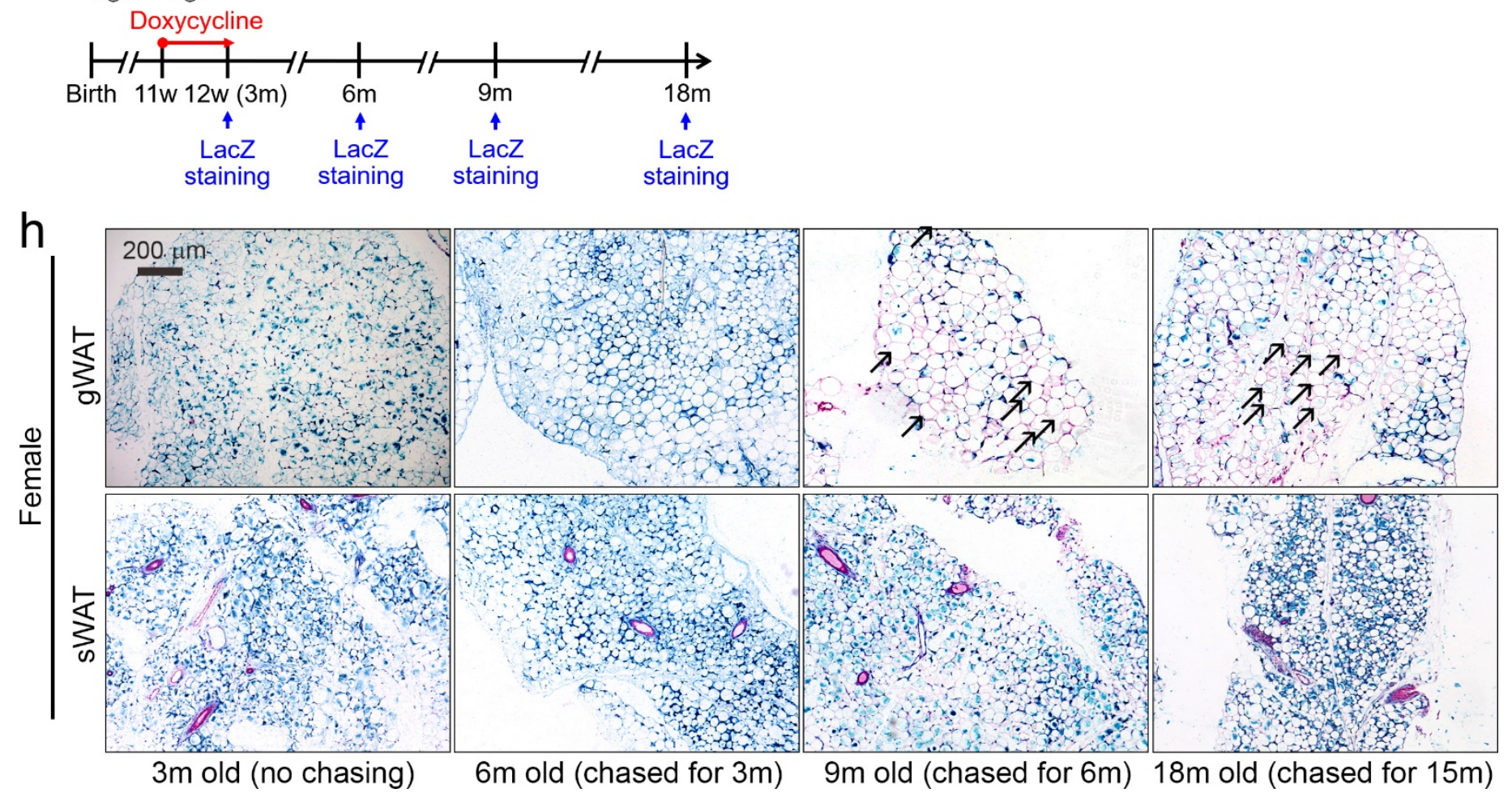


\section{Extended Data Fig. 2}

a

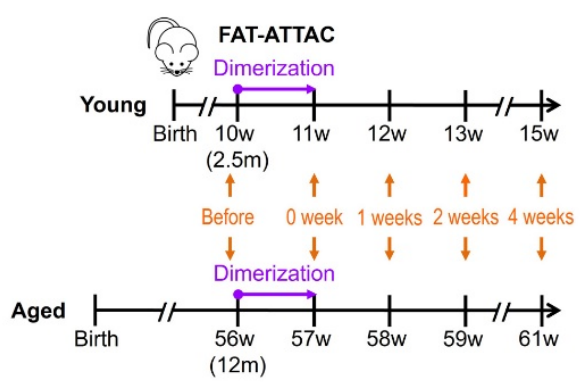

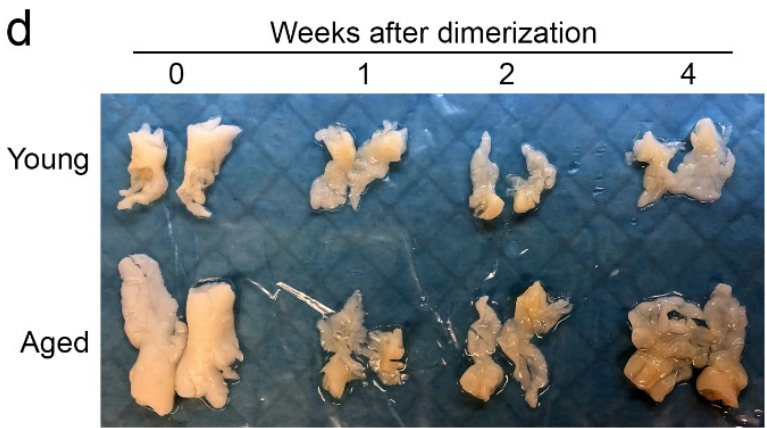

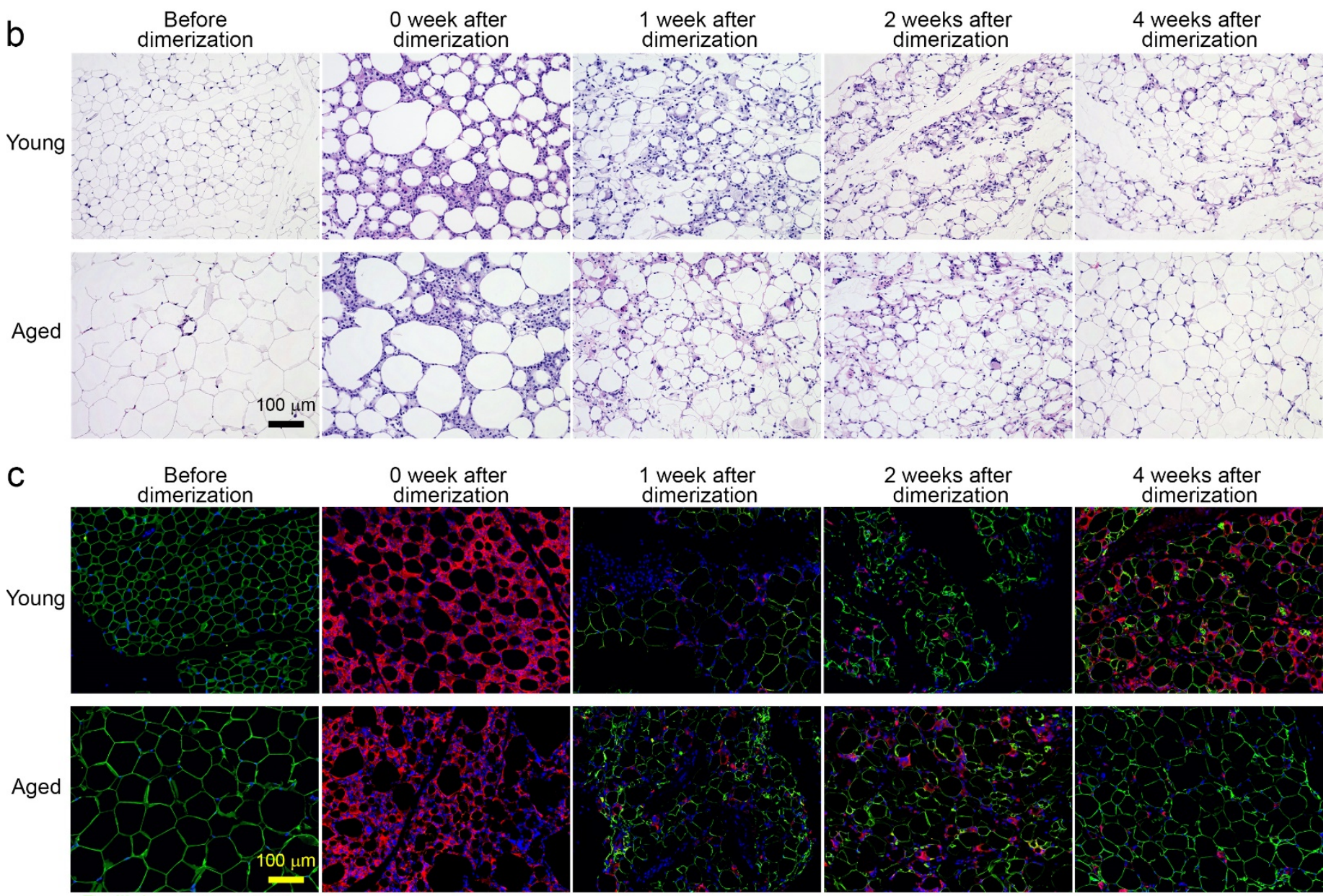




\section{Extended Data Fig. 3}
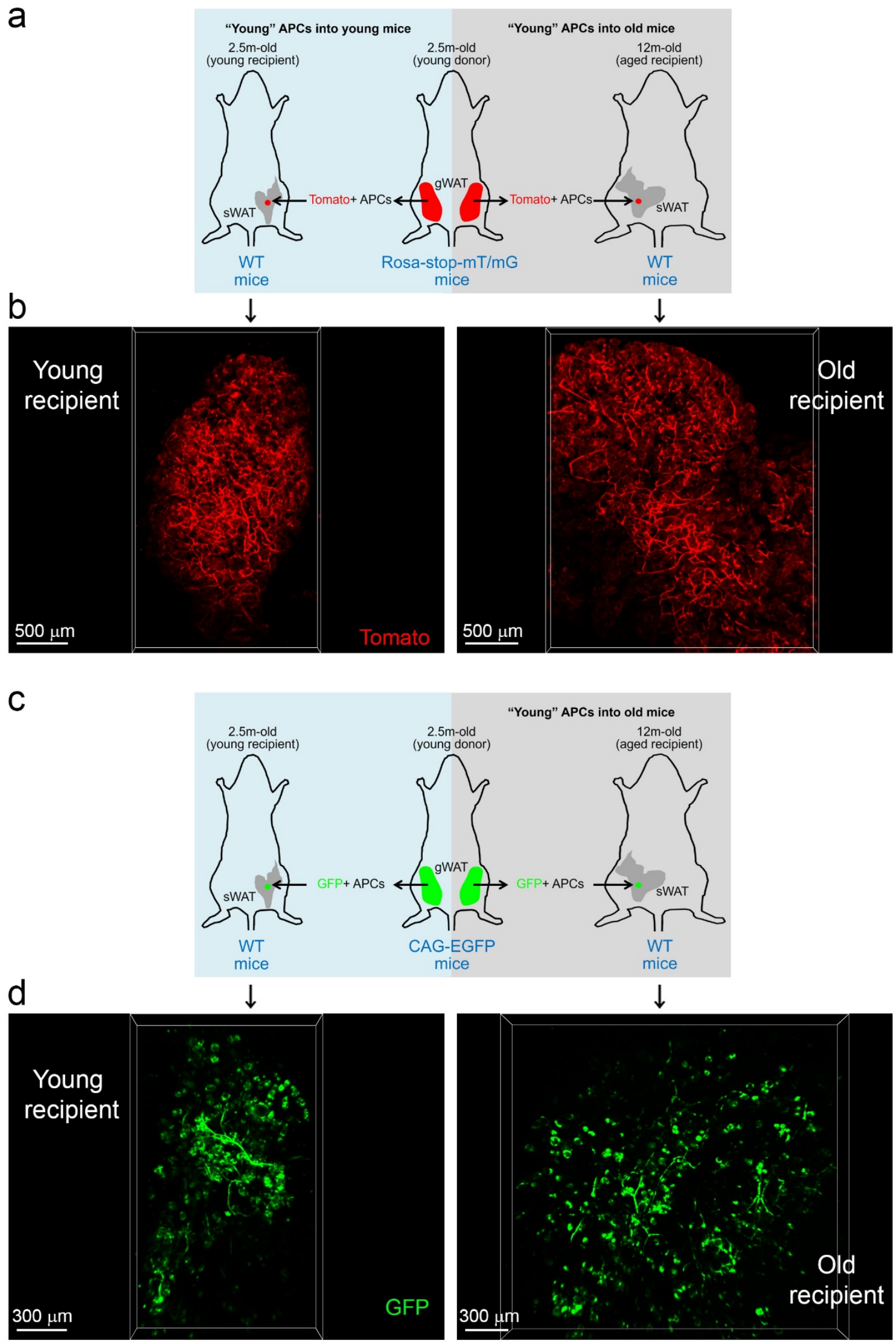


\section{Extended Data Fig. 4}
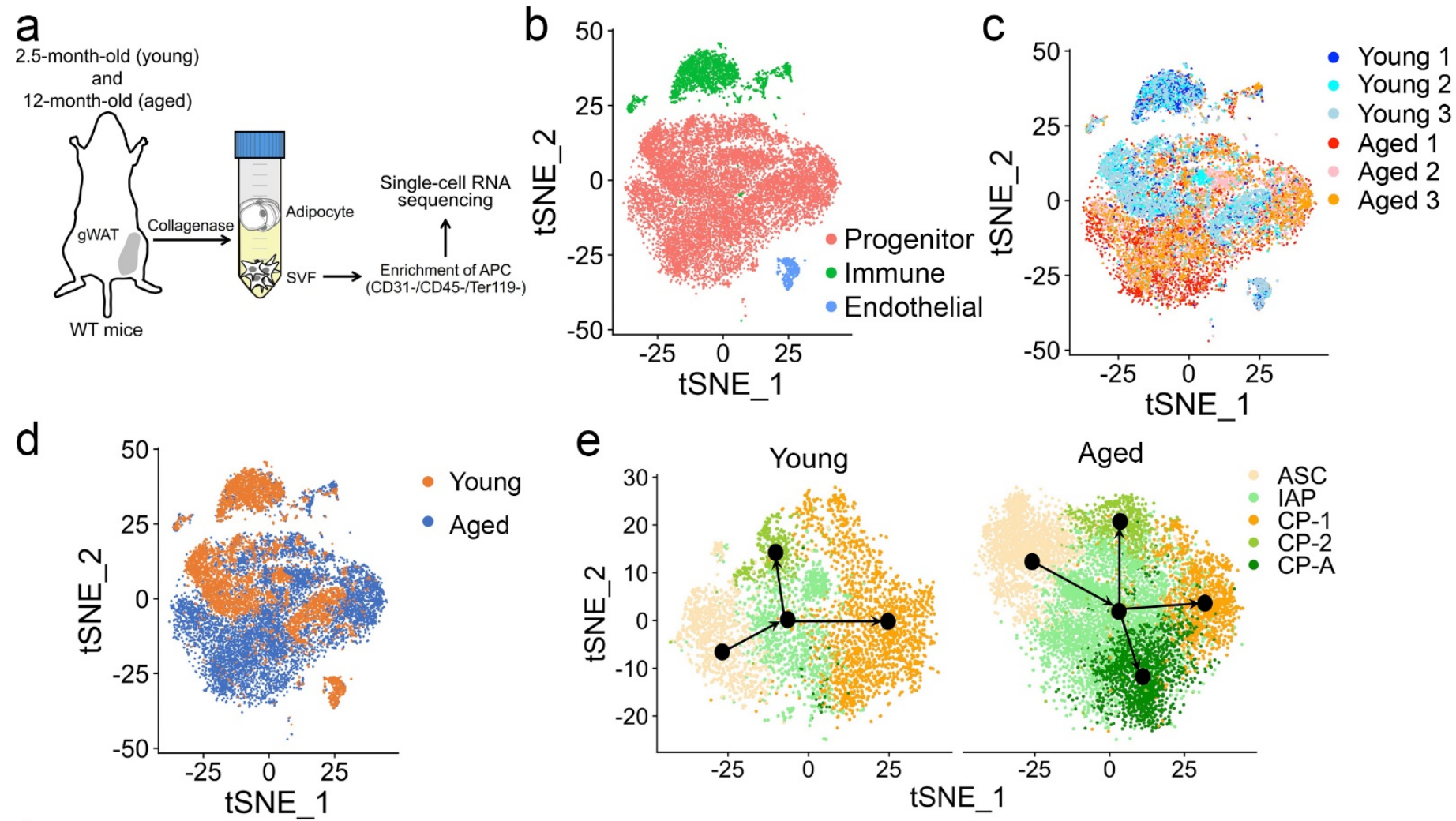

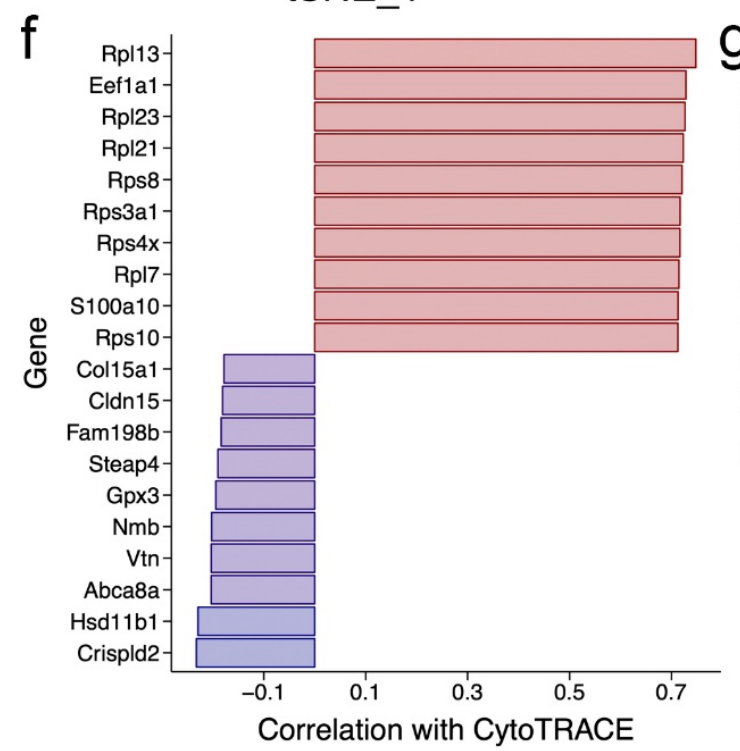

h

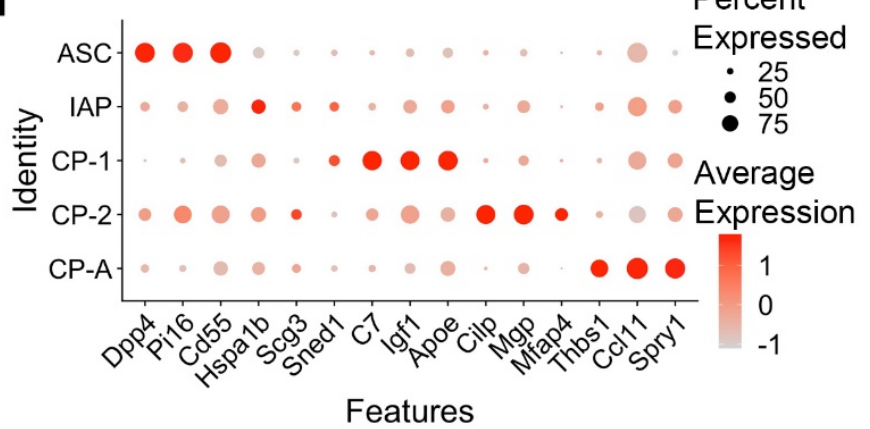

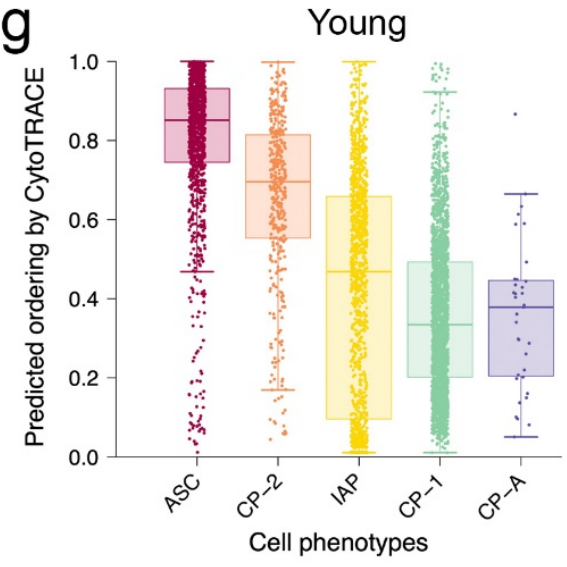
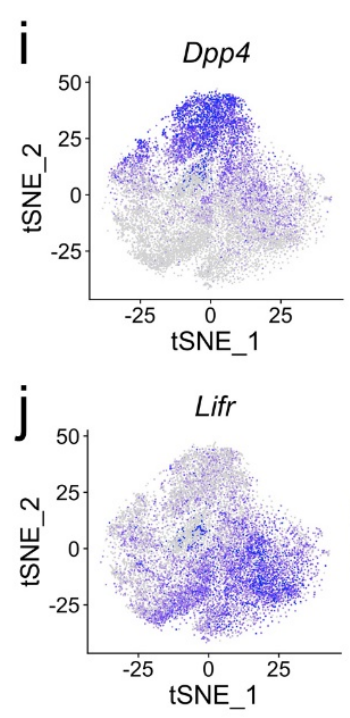
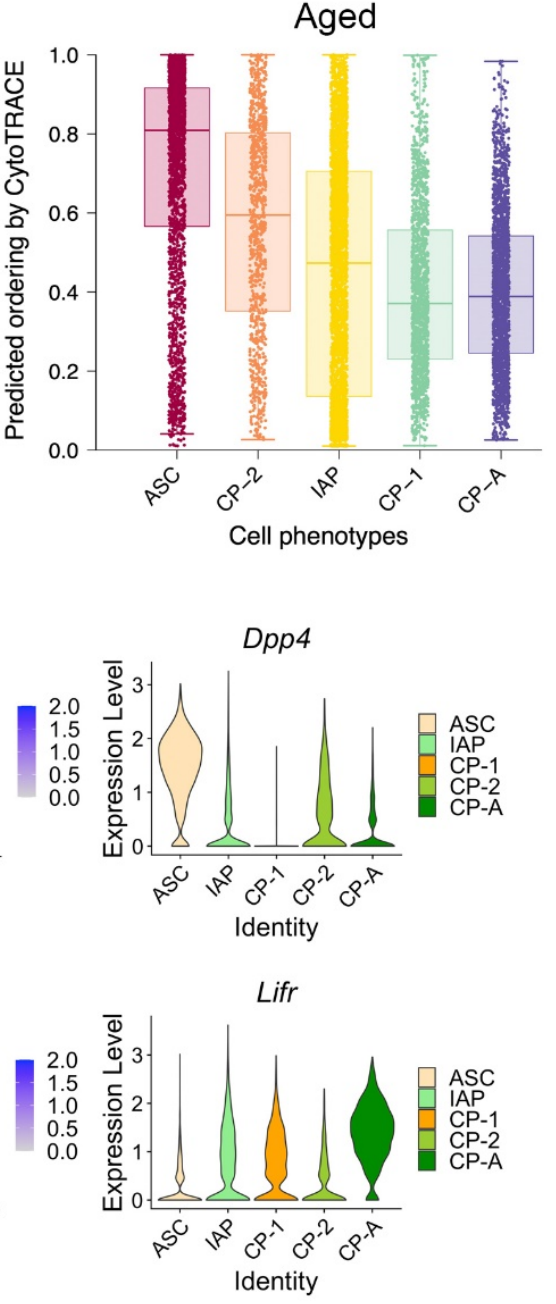


\section{Extended Data Fig. 5}

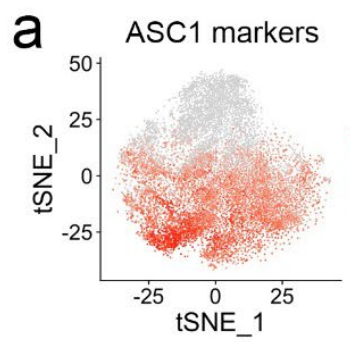

C FAP1 markers

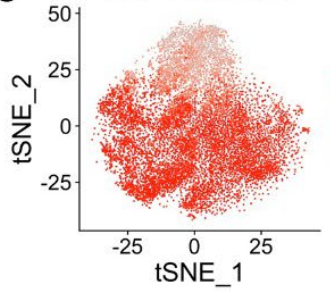

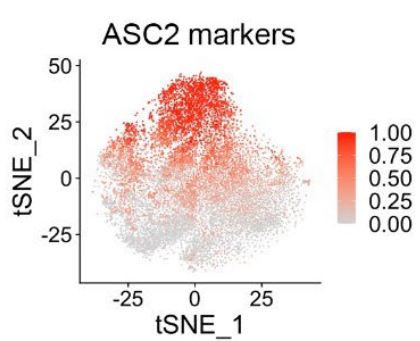

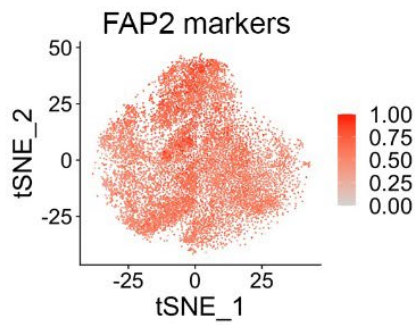

d

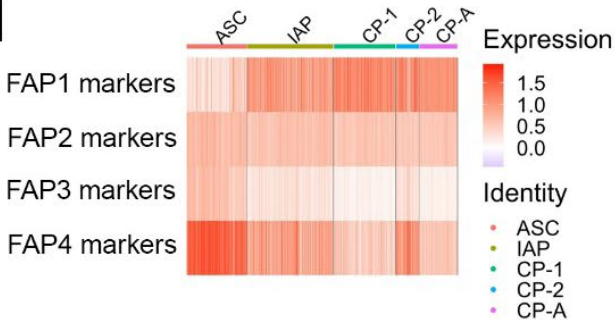

e
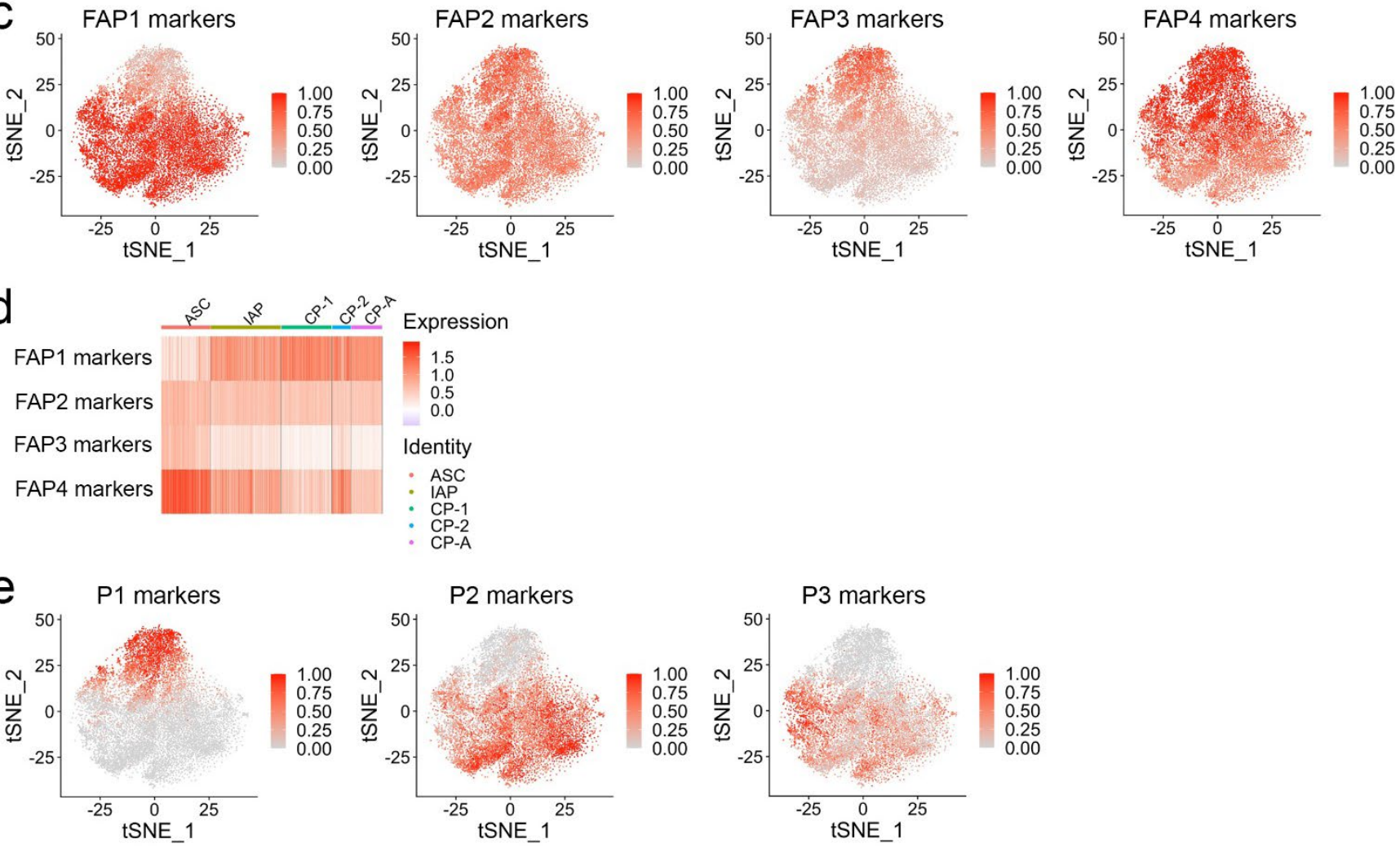
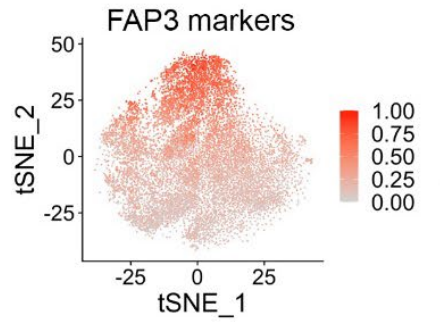

b

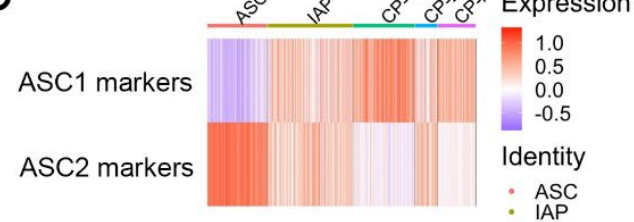

- IAP

- CP-1

- CP-2

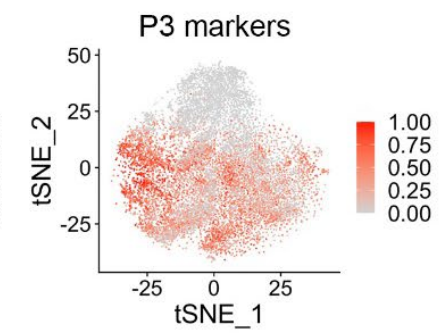

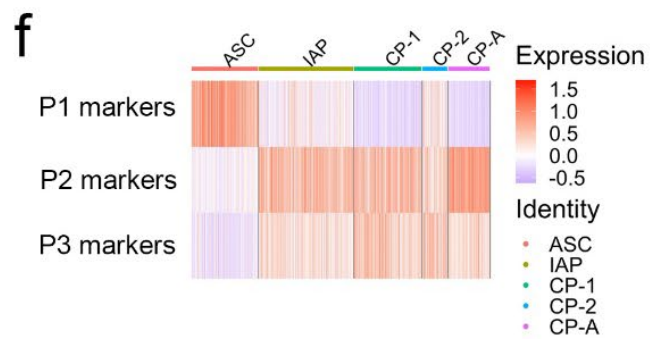




\section{Extended Data Fig. 6}
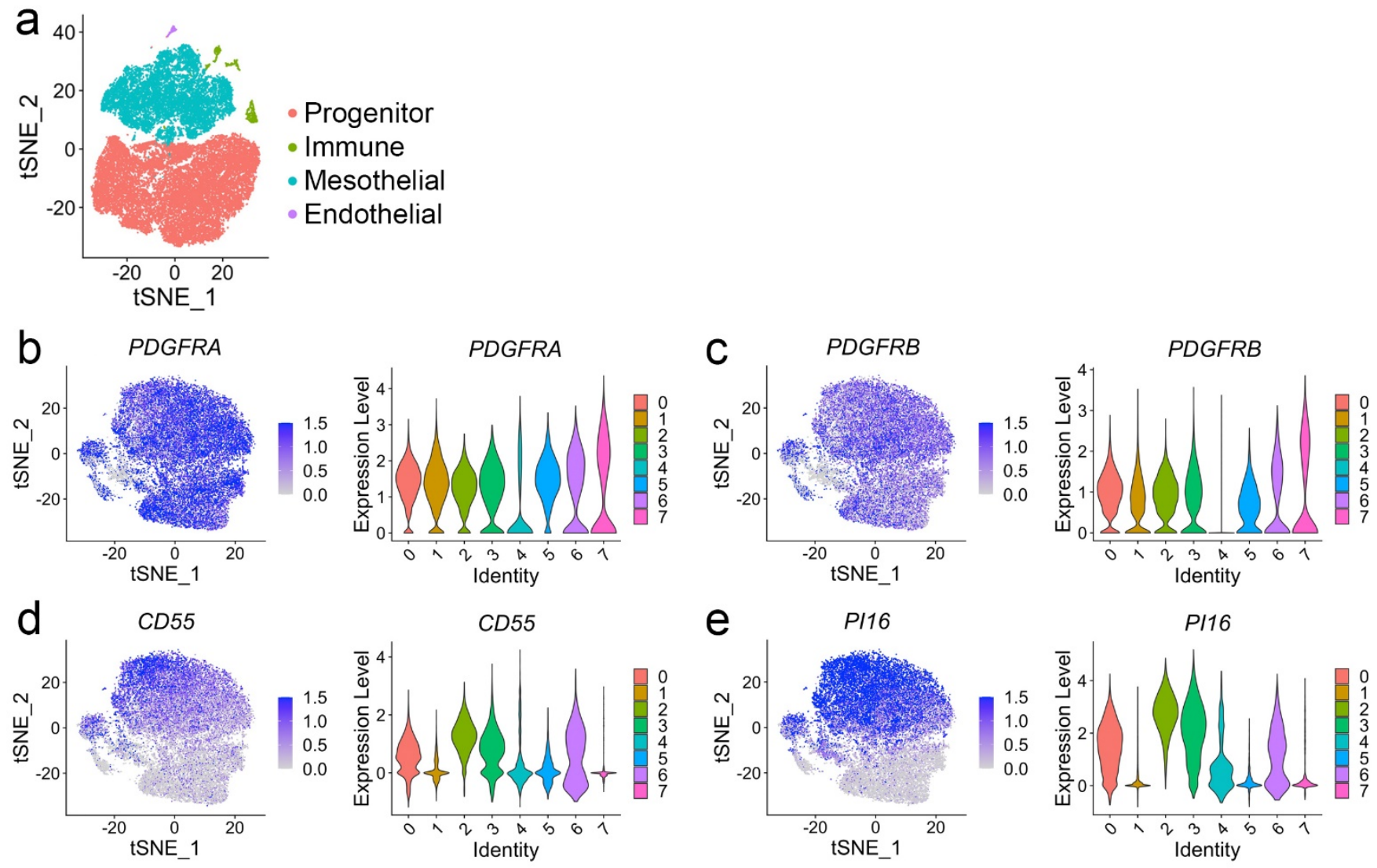

PI16

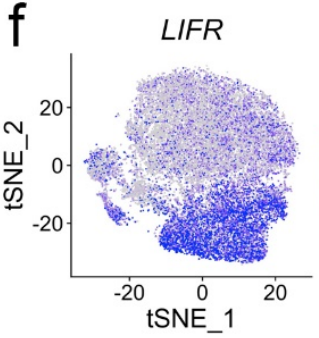

LIFR

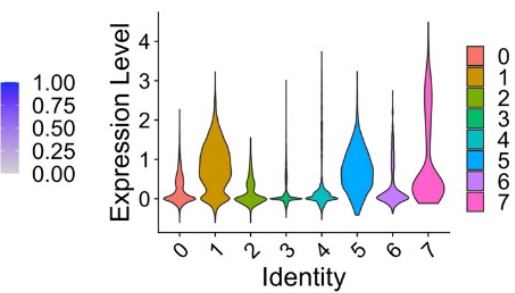

g
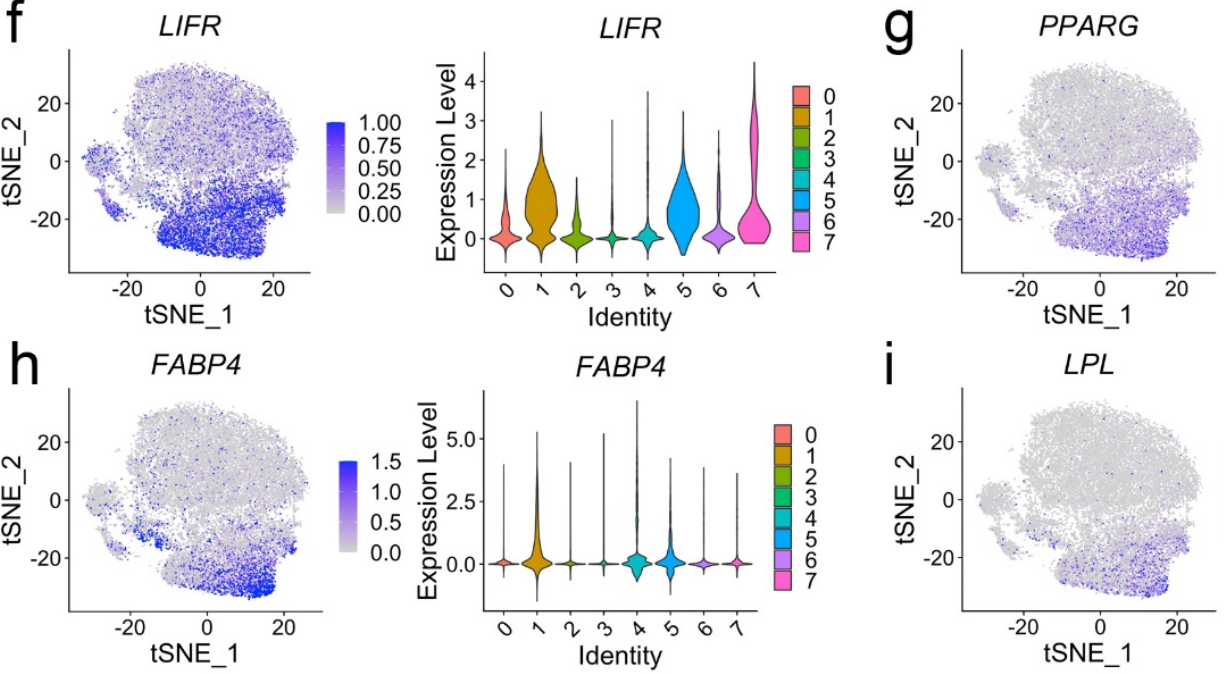

FABP4
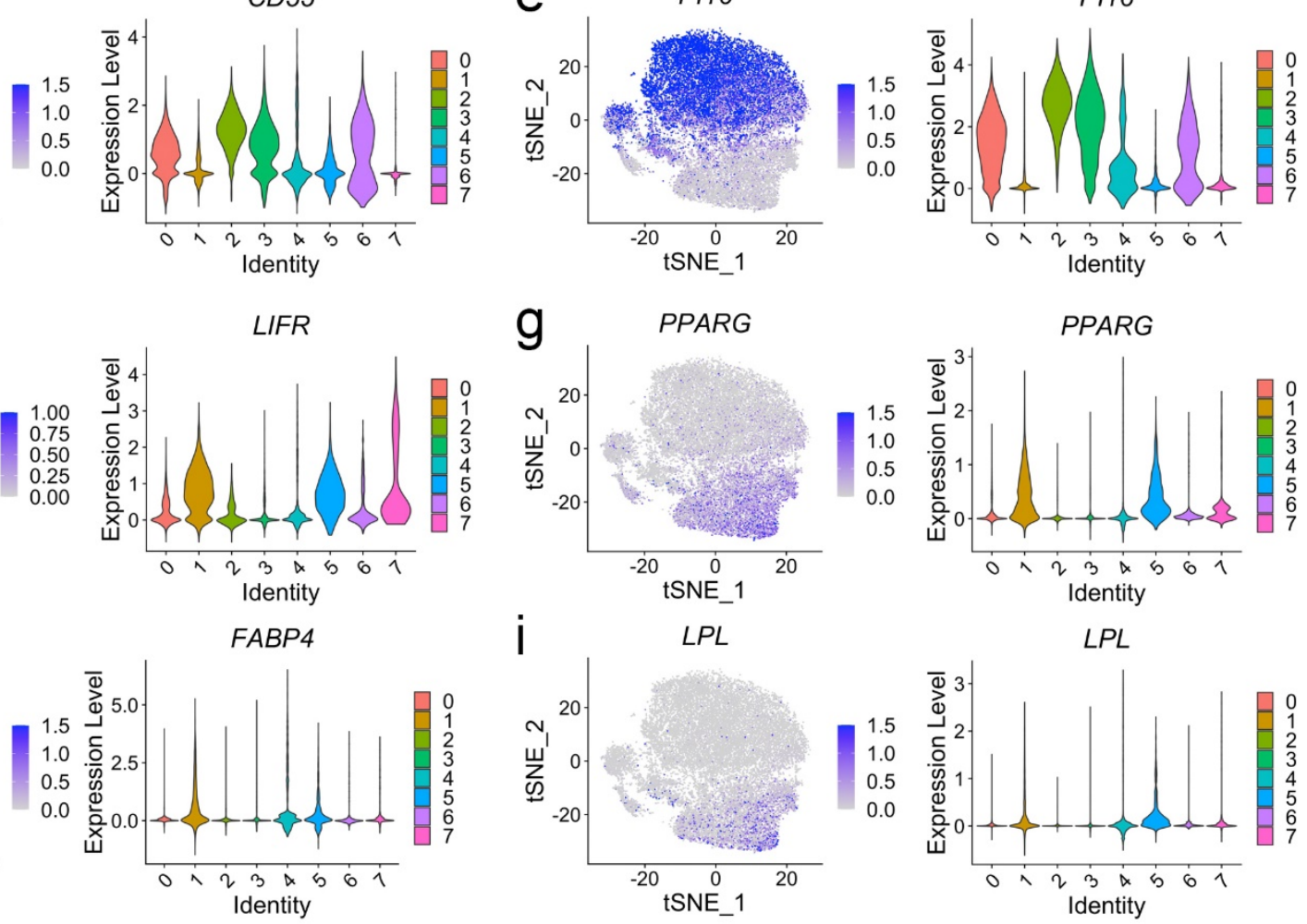

PPARG

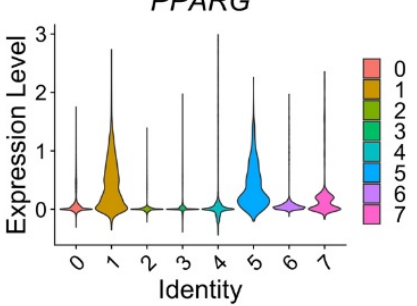

$L P L$

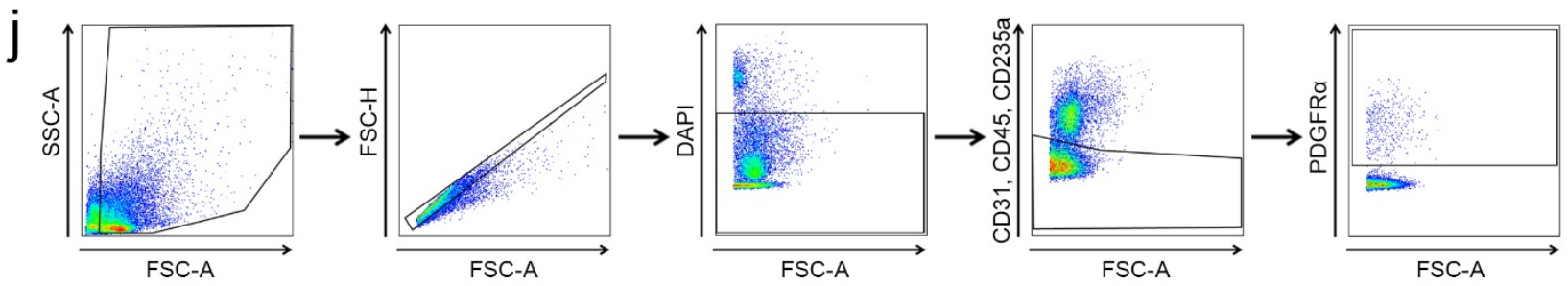




\section{Extended Data Fig. 7}

a Complete cocktail added $3 \mathrm{~d}$ post seeding
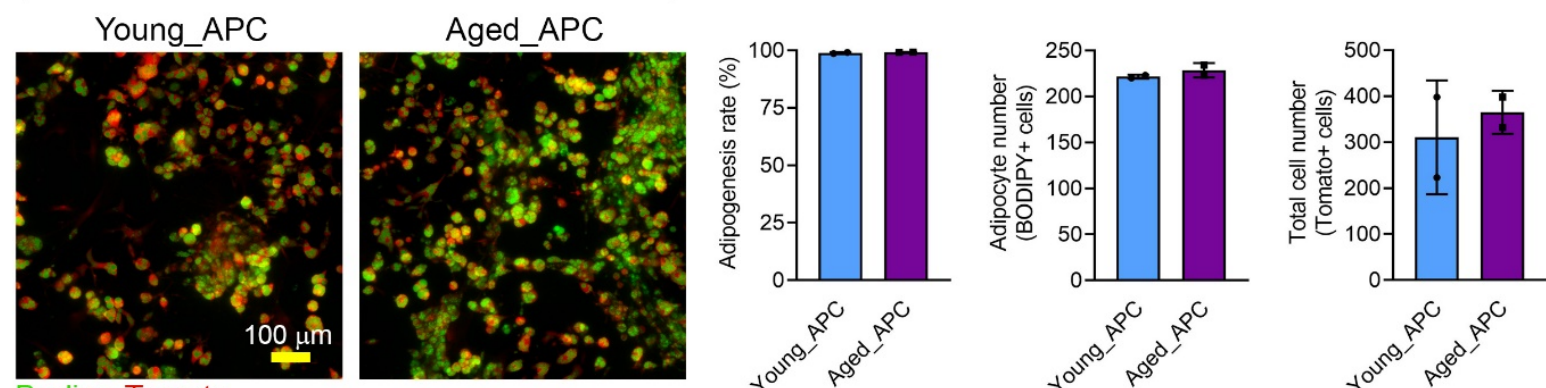

Bodipy Tomato
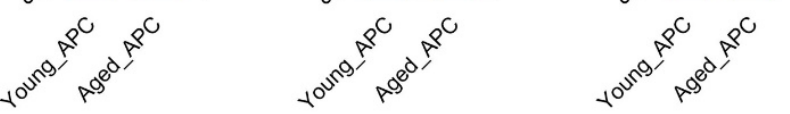

b Adipogenic cocktail added $16 \mathrm{~h}$ post seeding
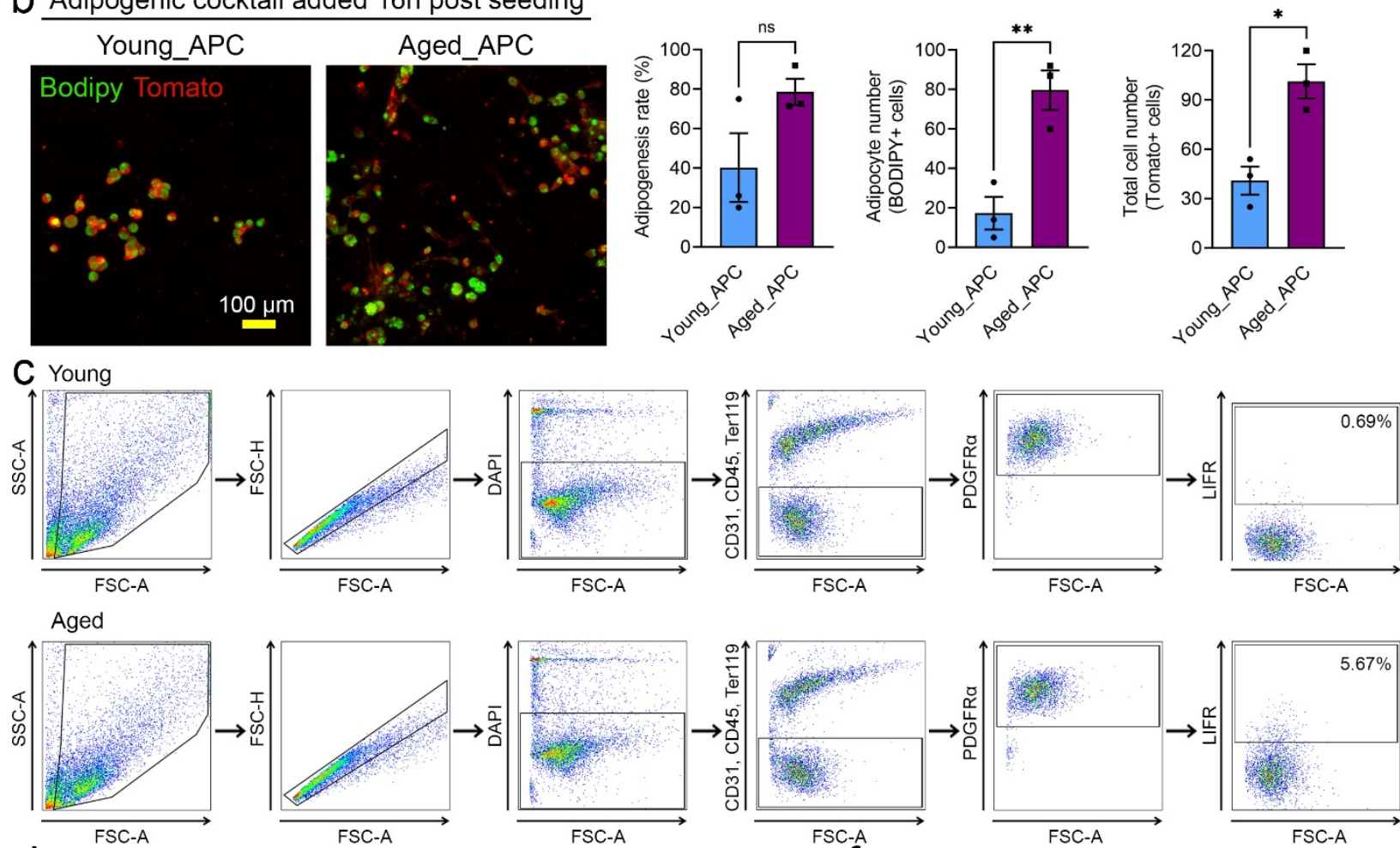

d

ASC Beads selection
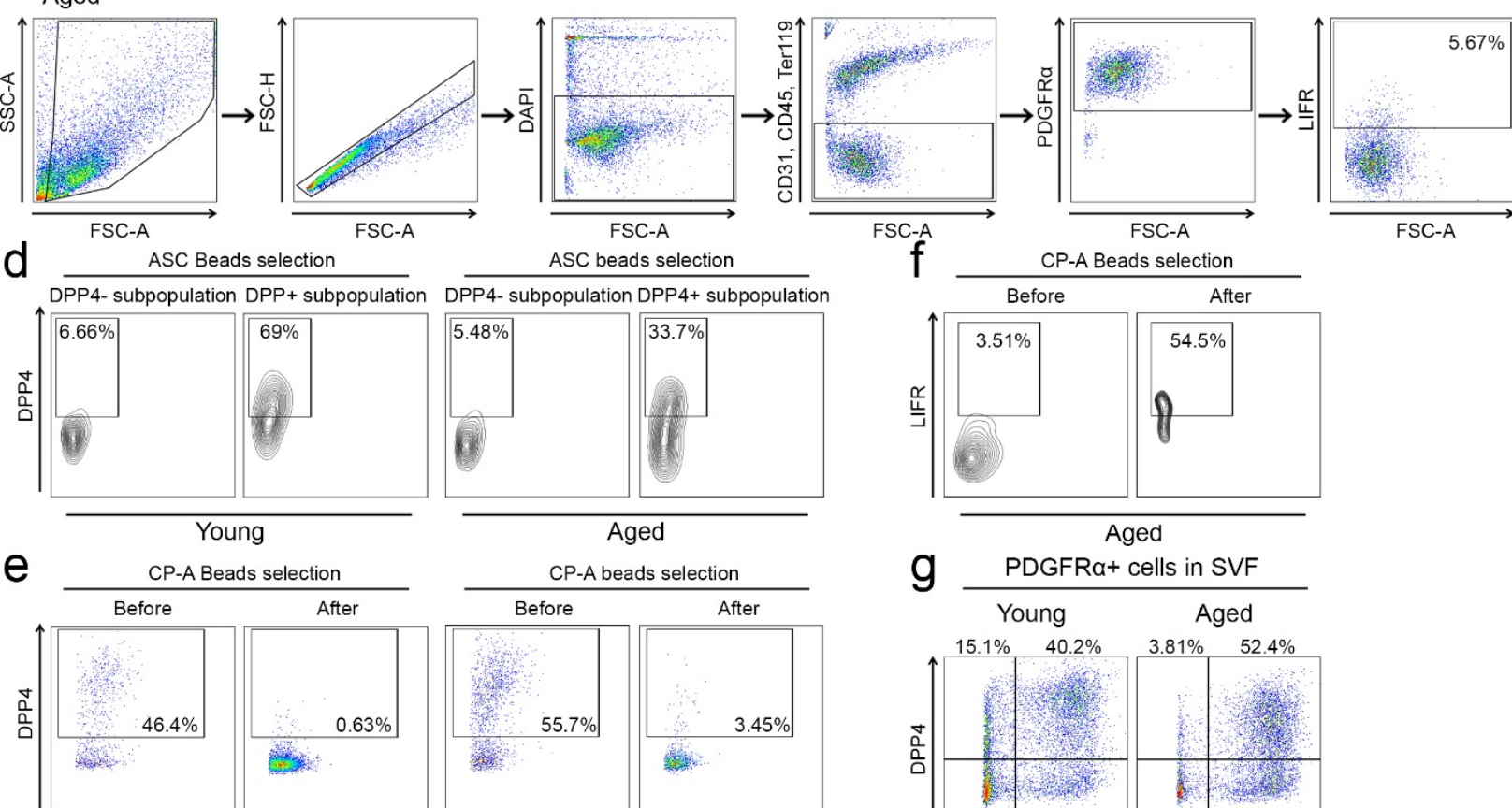

CP-A Beads selection

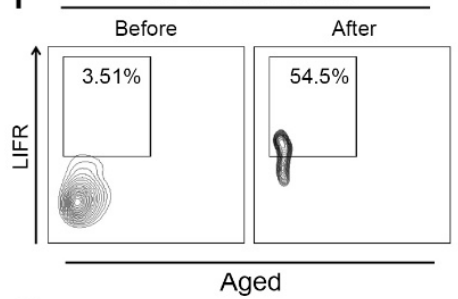

e

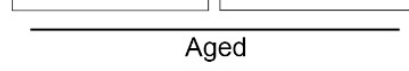

g
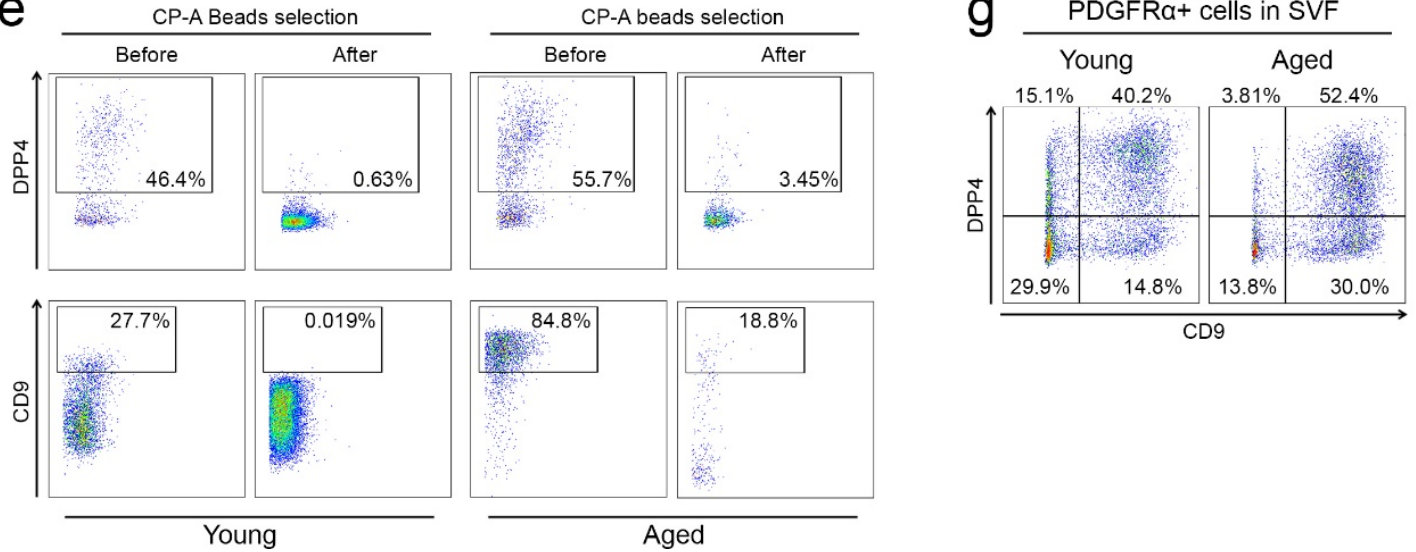


\section{Extended Data Fig. 8}

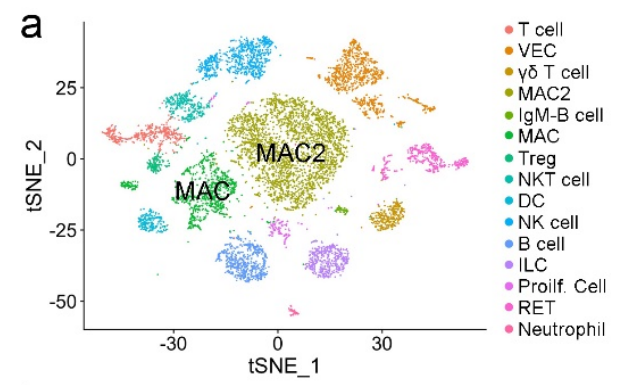

b

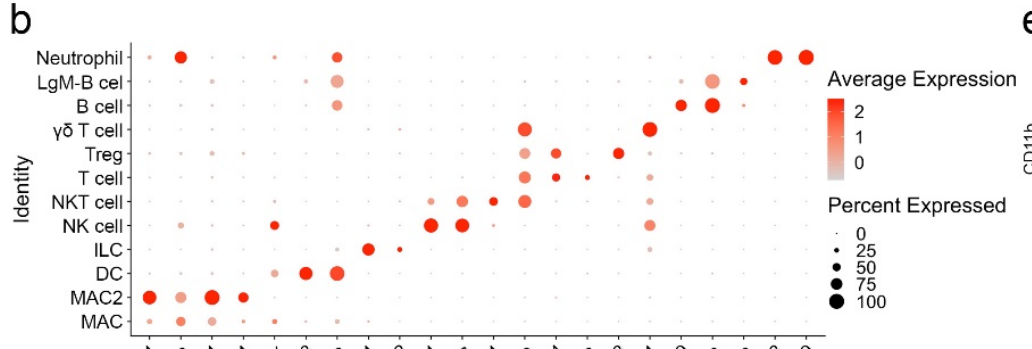

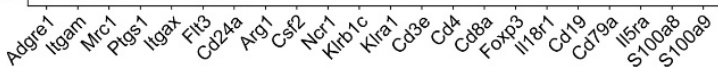
Features
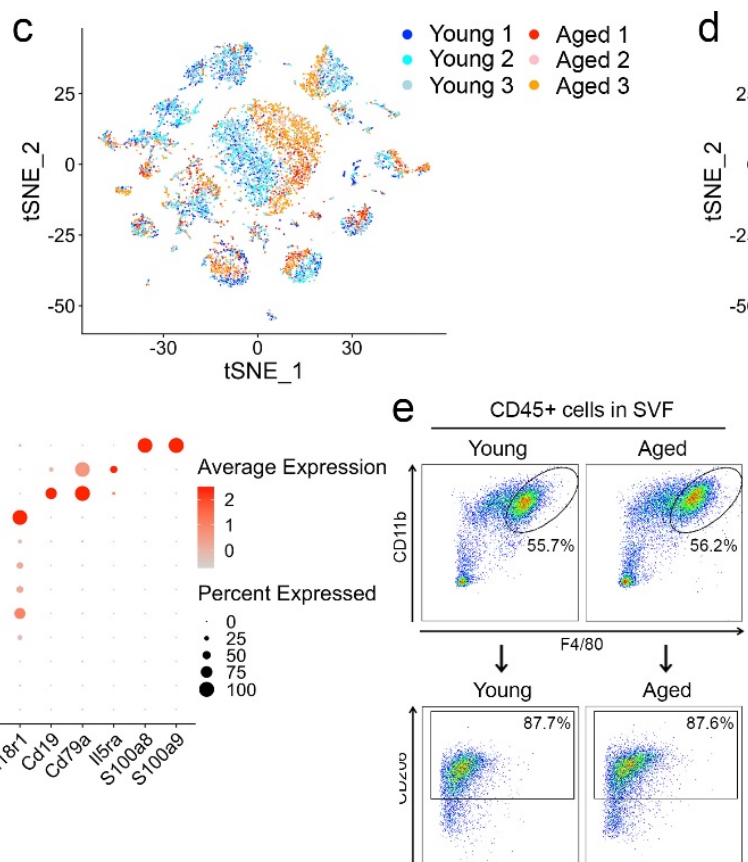
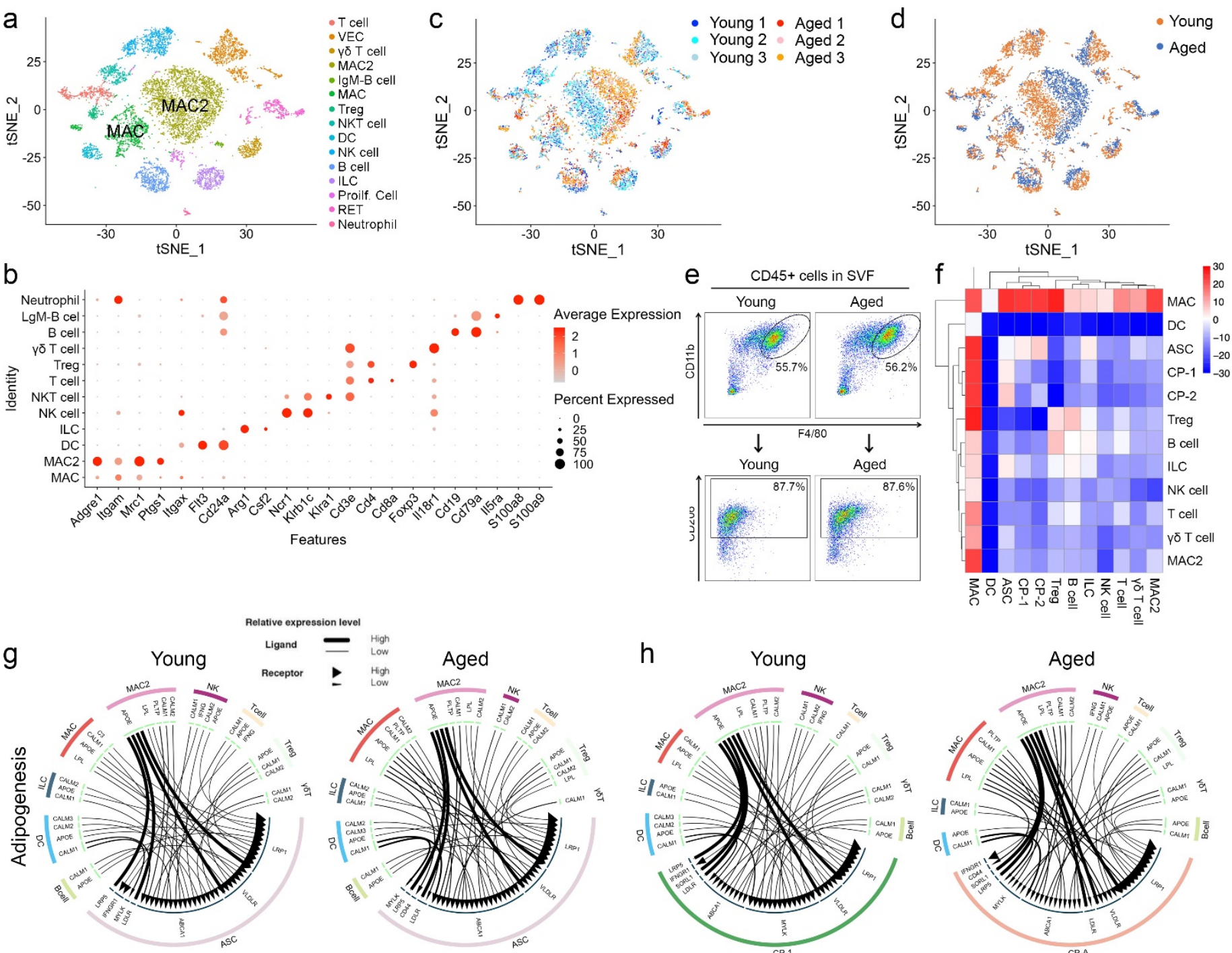

h
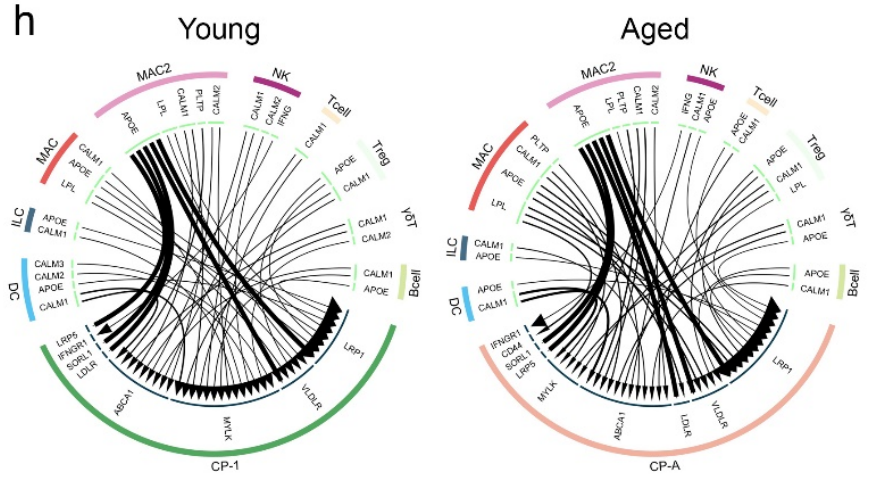

i
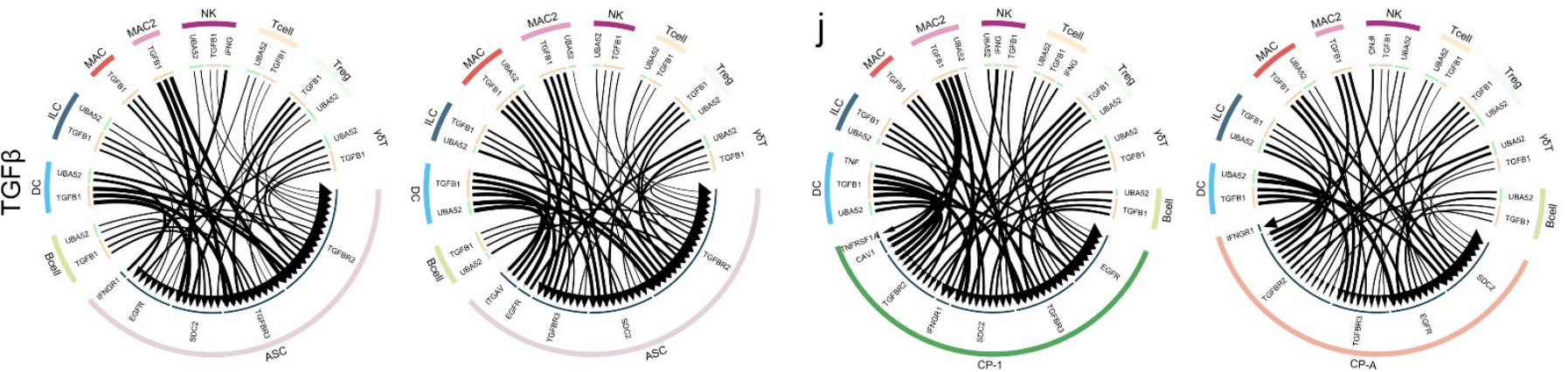

k
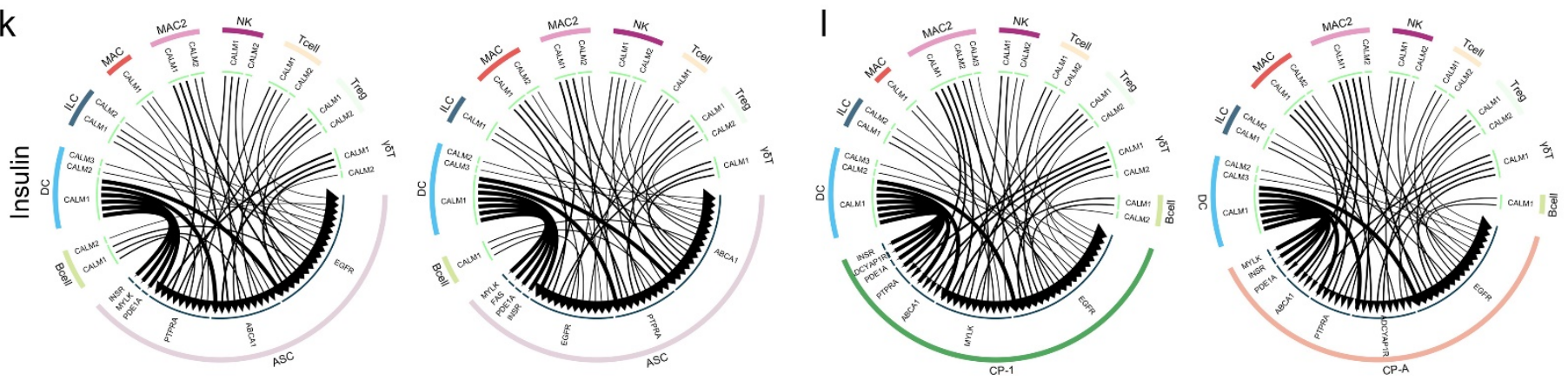
Extended Data Fig. 9
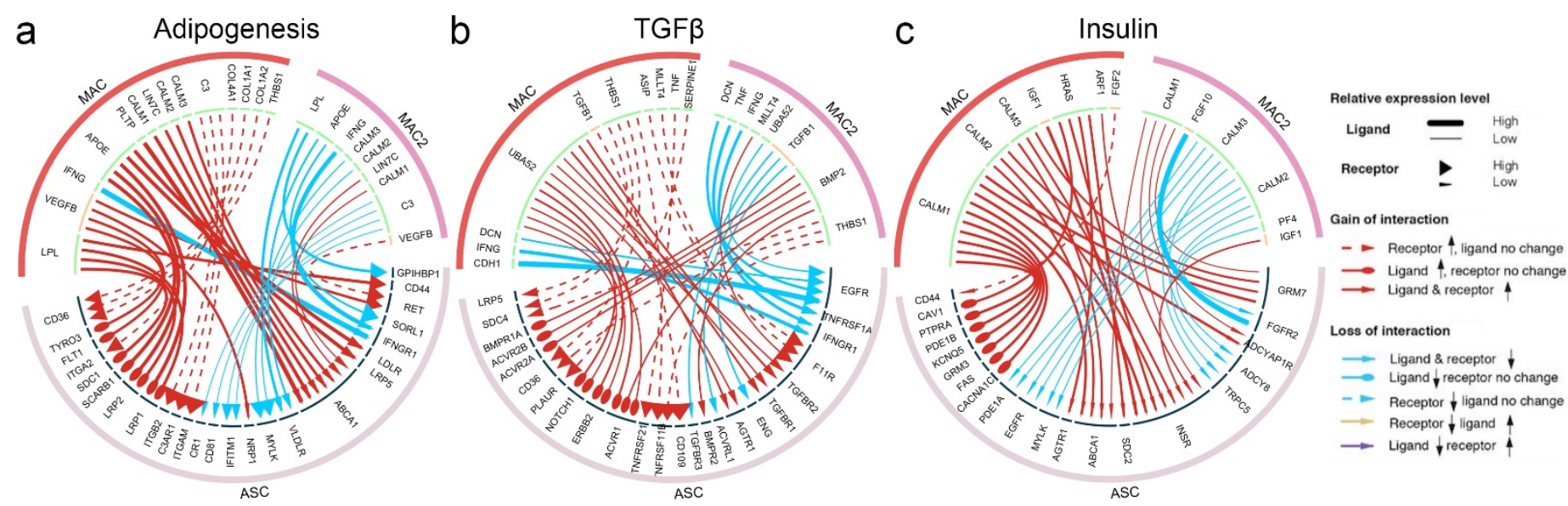

d

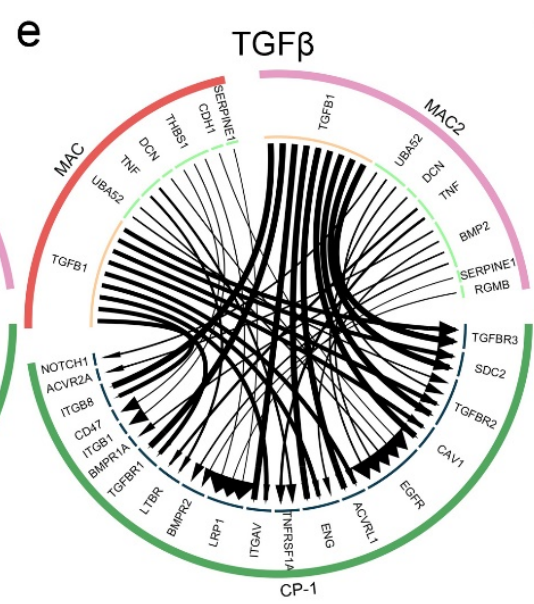

f
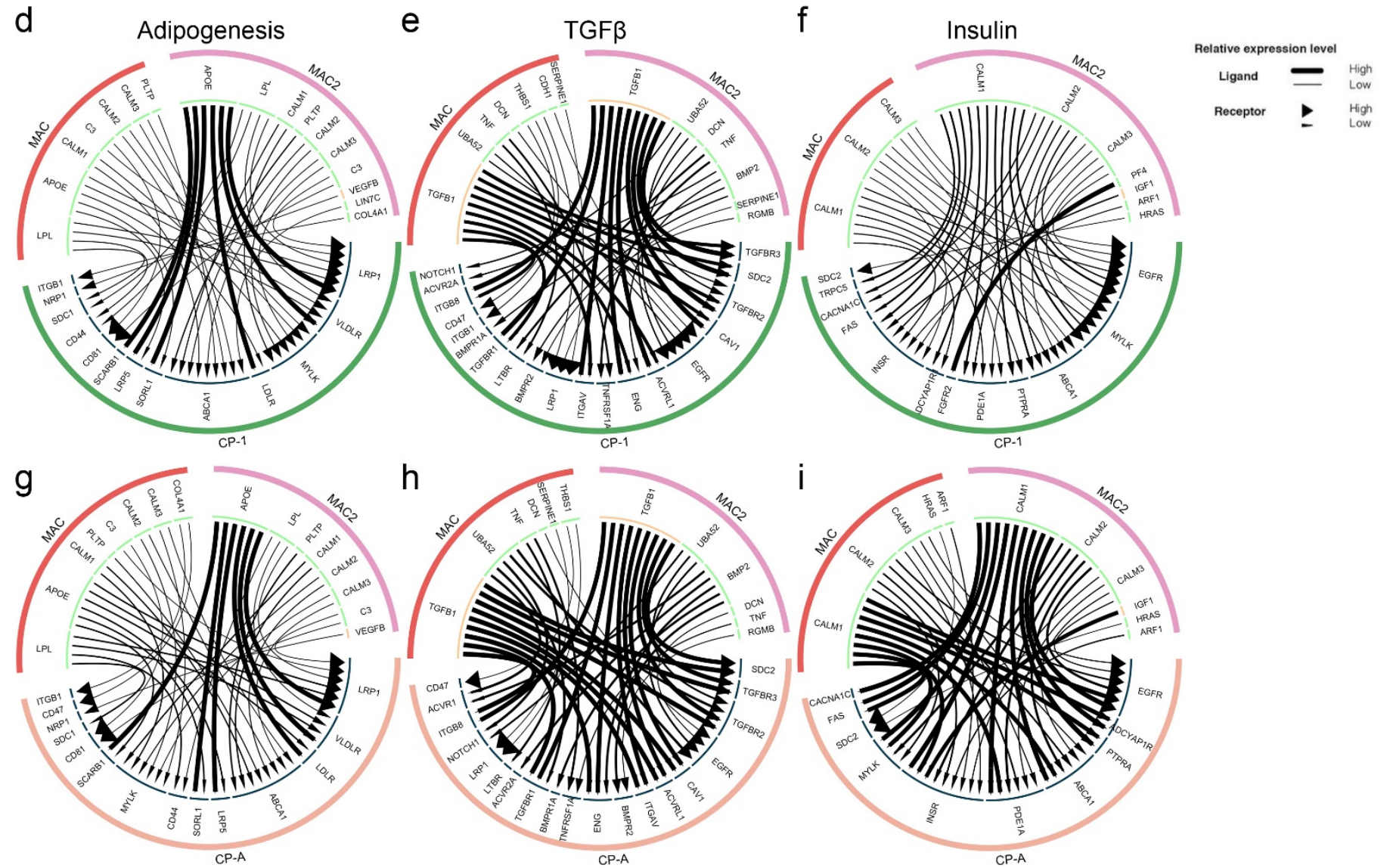


\section{Extended Data Fig. 10}

a Rosa-stop-mT/mG
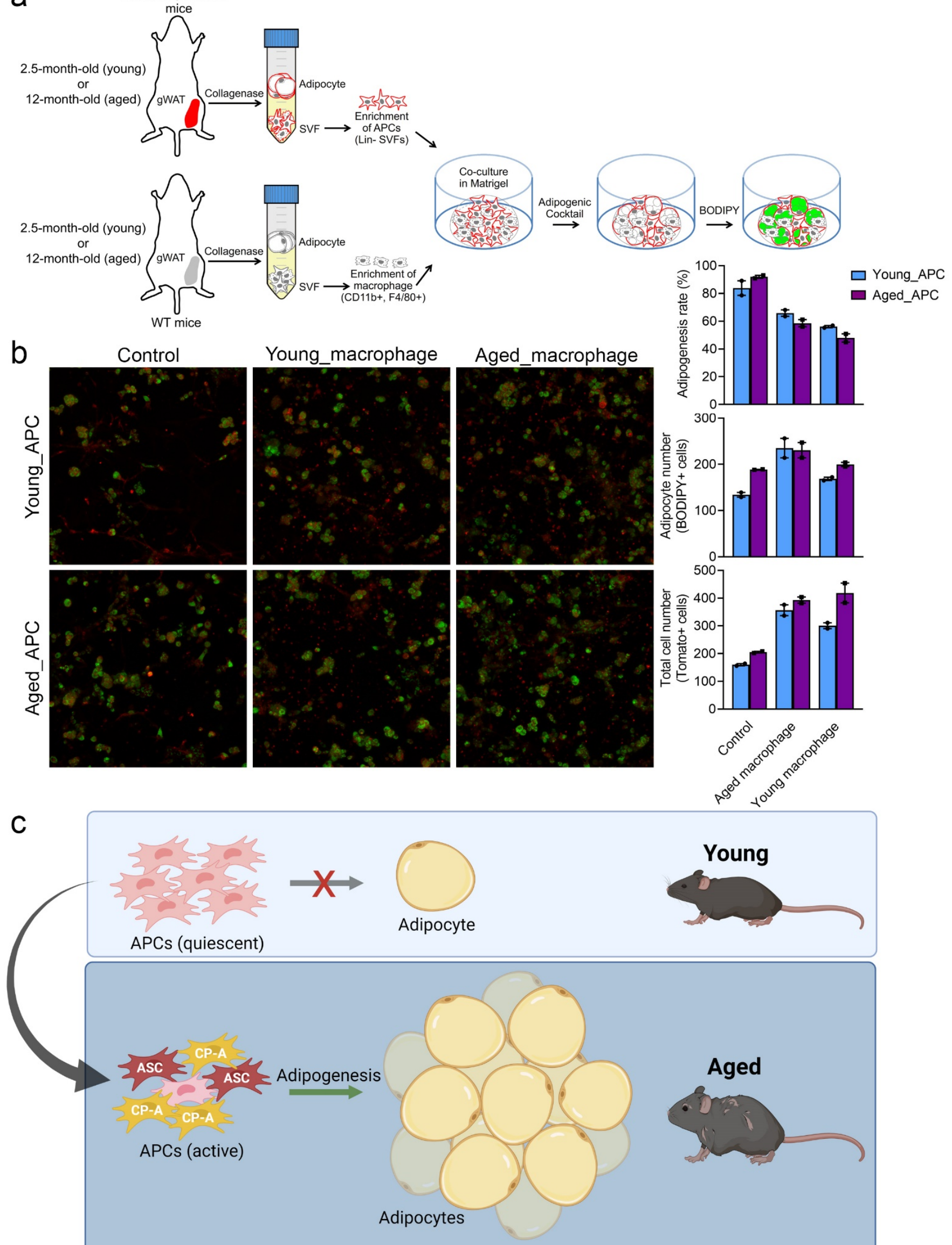


\section{Supplementary Files}

This is a list of supplementary files associated with this preprint. Click to download.

- Extendeddatavideoandtablelegend2.docx

- ExtendedDataTable1ASCAgeVSYoungpathway.xIsx

- ExtendedDataTable2AgemarkerCPApathway.xIsx

- Video1doublecolor.mp4

- Video2tomatoyoungtoyoung.mp4

- Video3tomatoagedtoyoung.mp4

- Video4YgRecipient.mp4

- Video5AgeRecipient.mp4

- Video6Ygrecipient.mp4

- Video7Agerecipient.mp4

- Video8YgDPP4.mp4

- Video9AgeDPP4.mp4

- Video10CP1.mp4

- Video11CPA.mp4 